\title{
THESIS
}

\section{EQUINE FECAL MICROBIOTA CHANGES ASSOCIATED WITH ANTHELMINTIC ADMINISTRATION}

\author{
Submitted by \\ Isabelle Kunz \\ Department of Animal Sciences \\ In partial fulfillment of the requirements \\ For the Degree of Master of Science \\ Colorado State University \\ Fort Collins, Colorado
}

Summer 2018

Master's Committee:

Advisor: Stephen Coleman

Tanja Hess

Diana Hassel

Jessica Metcalf 
Copyright by Isabelle Kunz 2018

All Rights Reserved 


\begin{abstract}
EQUINE FECAL MICROBIOTA CHANGES ASSOCIATED WITH ANTHELMINTIC ADMINISTRATION
\end{abstract}

The equine gastrointestinal tract contains a complex ecosystem comprised of microorganisms and intestinal helminth parasites. Increasing evidence suggests that horses rely on their gastrointestinal microbial populations for many aspects of metabolic and immune function. Furthermore, interactions between the microbes, helminths, and their host may impact these functions, which are vital to maintaining gastrointestinal homeostasis. The use of anthelmintic drugs is a common practice of intestinal helminth parasite control in domestic horse health management. The use of anthelmintic drugs is very effective for controlling the burden of parasitic infection and associated clinical signs, however anthelmintic administration has also been associated with gastrointestinal disturbances in the horse. The potential effects of anthelmintic drug administration on the equine gastrointestinal microbiota and its' role in homeostasis is not yet understood.

The objective of research presented in this thesis is to investigate the impact of anthelmintic administration on the equine gastrointestinal microbiota. This goal was addressed in two main studies, which used 16S rRNA amplicon sequencing to identify and describe the microbial populations present in equine fecal samples. The first study described is a pilot project which aimed to characterize the impact of anthelmintic treatment on the fecal microbiota of horses without an observable helminth infection. The results of this study indicated that subtle differences in the microbial community composition and structure are detectable between samples collected before 
and after anthelmintic treatment. A second study was then conducted as a follow-up to the pilot which included horses with varying detectable levels of helminth infection as determined by fecal egg counts. Results of the second study further demonstrated microbiota changes following anthelmintic treatment, while also suggesting that the most notable effects of anthelmintic treatment may be observed in fecal samples between 48 and 72 hours post-treatment. The results of these studies suggest that anthelmintic treatment may be associated with changes in the equine fecal microbiota. 


\section{ACKNOWLEDGEMENTS}

Growing up, I was one of the few children that always provided the same answer when asked, “what do you want to be when you grow up?". It wasn't until my last year at the University of Kentucky that my answer changed and I realized that I no longer aspired to be an equine veterinarian. Instead, I found myself yearning to be on the front lines of research and discovery. This sudden change occurred while sitting in the front row of a sparsely occupied auditorium, listening to Dr. Ernest Bailey describe his role in the advancement of Equine Genetics. I had never been so engaged in a course and stayed after every class to bug Dr. Bailey with hundreds of questions about each and every topic he presented. After one of these conversations, I recall bursting through the doors of my undergraduate advisor's office and proclaiming that I was going to be an equine geneticist, not a veterinarian. Little did I know that my path over the next few years would be fundamentally shaped by the inspiration of Dr. Ernest Bailey and the unwavering support of my advisor, Dr. Robert Coleman. So first, I would like to thank the two mentors that motivated me to continue on my quest for knowledge and who saw my potential even when I could not; Dr. Robert Coleman and Dr. Ernest Bailey. Had it not been for you, I would not have made it here today.

To Dr. Stephen Coleman, for taking a chance on a bewildered horse girl and for not being scared off by my father. Thank you so much for the countless opportunities you provided me to learn and grow as an individual. Your approaches may not have always been conventional, but the lessons they taught me will never be forgotten. More than anything, thank you for always sticking by me through the ups as an advisor and as a friend. 
To Dr. Tanja Hess, Dr. Diana Hassel, and Dr. Jessica Metcalf for providing indispensable advice, guidance and support throughout my research and for their commitment to providing extraordinary mentorship even under the most trying circumstances.

To my lab partner that has become one of my closest friends, Kailee Reed. Your moral support, dark humor, and determination got me through the toughest times of the past few years. Thank you for always being there when I needed you. Please don't forget about me when you've won your noble prize.

To my friends and colleagues in the CSU Animal Breeding and Genetics group for your assistance, comradery, Christmas parties, and support. I would also like to thank Maggie from Meat Science and the QIIME2 help forum, for providing invaluable help and guidance when nobody else seemed to know the answers.

To Kevin, who always helped me to quench my thirst for adventure while ensuring that I didn't lose sight of my motivation. I will always be eternally grateful for your support through the last two years.

Finally, and most importantly, thank you to my parents and sister. Your enduring encouragement has allowed me to overcome the toughest of times. It is a credit to you all that I have been able to continue to pursue my dreams with question if you will be standing behind my decisions to do so. You have molded me into the person I am today by allowing me to experience things in life that most people only dream of. I can never thank you enough for all of your love and support. So, it is with great gratitude that I dedicate this thesis to my parents, Tracey and Gregg, and my sister, Victoria. 


\section{TABLE OF CONTENTS}

ABSTRACT

CHAPTER II: CHARACTERIZING THE EFFECT OF ANTHELMINTIC ADMINISTRATION ON THE EQUINE FECAL MICROBIOTA IN HORSES WITHOUT AN OBSERVABLE

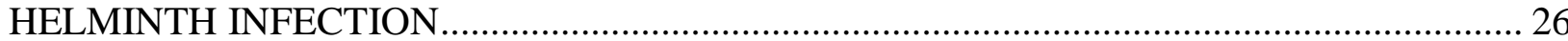

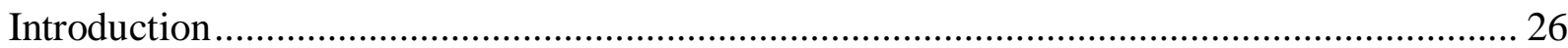

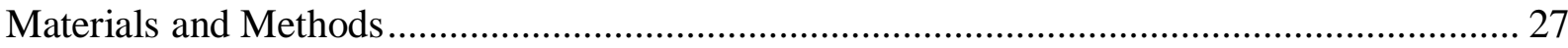

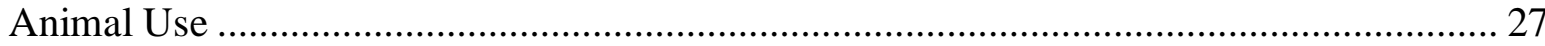

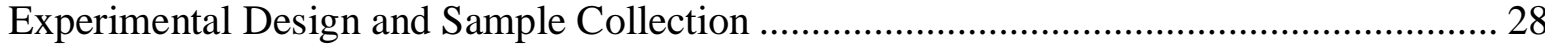

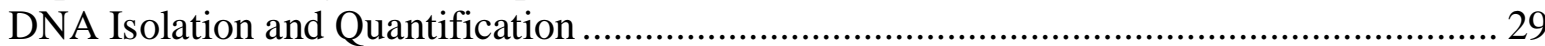

16S rRNA Amplification and Next-Generation Sequencing ............................................... 29

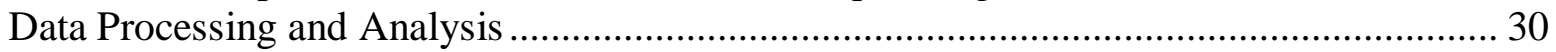

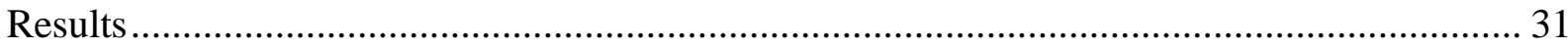

16S rRNA Amplicon Sequencing Data Summary ……………………………………....... 31

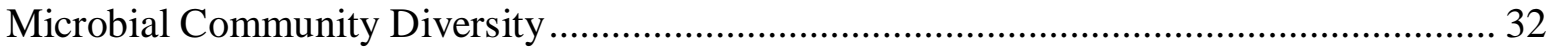

Microbial Community Composition .................................................................................. 34

Differential Abundance of Taxonomic Features Between Sample Collection Groups ........ 36

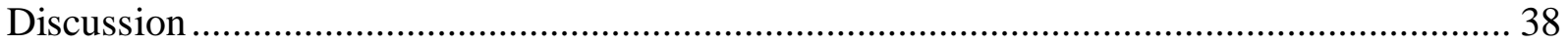

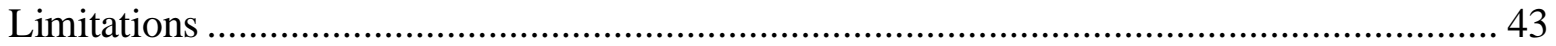

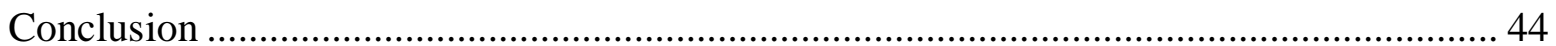

CHAPTER III: THE EQUINE FECAL MICROBIOTA ASSOCIATED WITH HELMINTH EGG SHEDDING LEVELS AND THE TEMPORAL IMPACT OF ANTHELMINTIC

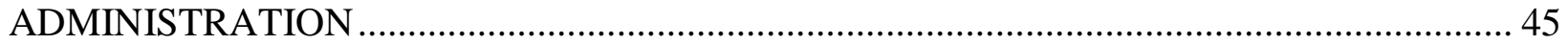

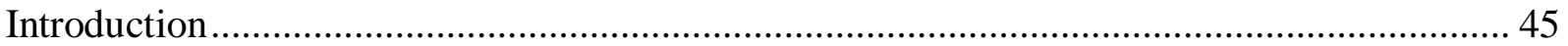

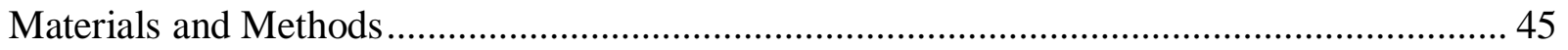

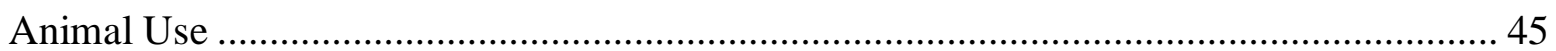

Experimental Design and Sample Collection ............................................................... 47

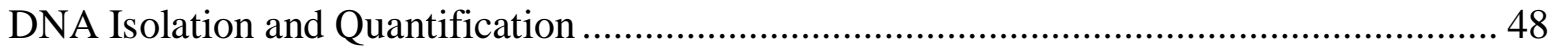

16S rRNA Amplification and Next Generation Sequencing ............................................. 48

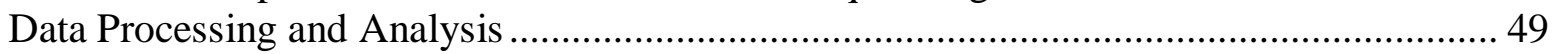

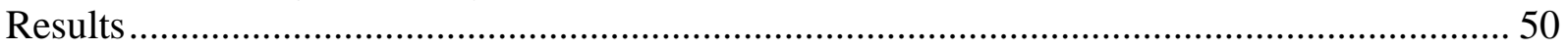

Fecal Egg Count Diagnostics Results Summary ………........................................... 50

16S rRNA Amplicon Sequencing Data Summary .......................................................... 51

Characterization of the Microbiota Between FEC Shedding Groups ................................... 52 
Temporal Variation of the Microbiota Following Anthelmintic Administration ................ 55

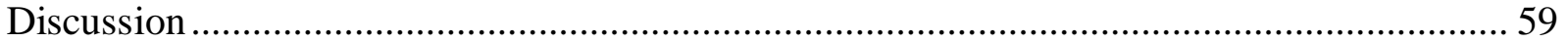

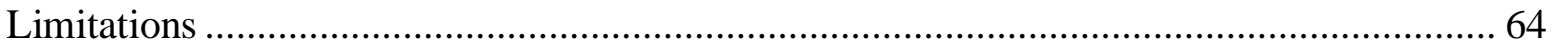

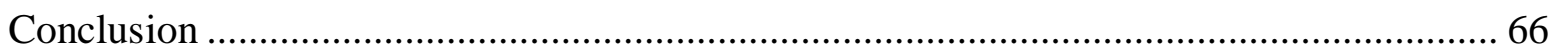

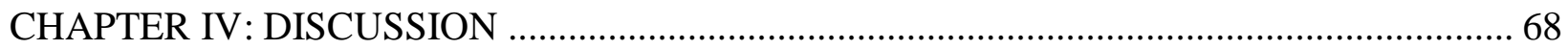

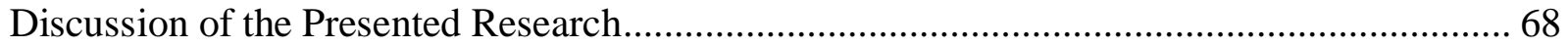

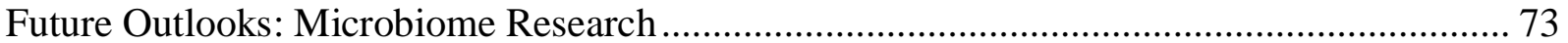

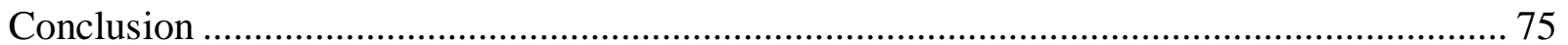

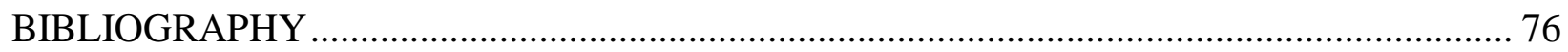

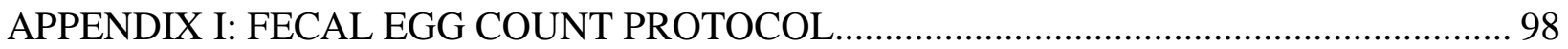

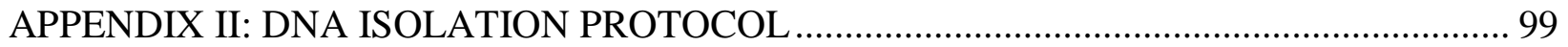

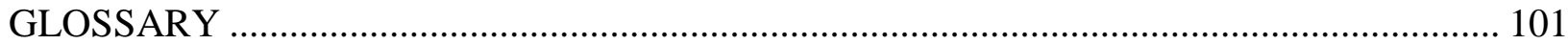

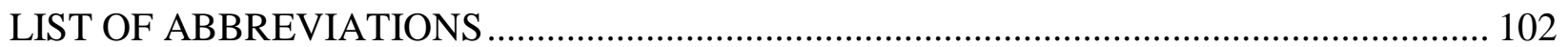




\section{CHAPTER I: LITERATURE REVIEW}

\section{The Equine Microbiota}

The term "microbiome" has quickly gained popularity and notoriety with the rise of research pertaining to earth's microscopic life. This term refers to the combined genetic material of all microorganisms within an environment which is often used interchangeably with the term "microbiota", which simply refers to all of the microorganisms in the particular environment (see

Glossary). The "host-associated microbiome", is further defined as a community of microorganisms requiring a host organism to sustain their symbiotic form of life $[1,2]$. The hostassociated microbiome has generated a large amount of interest from researchers focused on mammalian health and disease, as it plays many vital roles in the maintenance of homeostasis, among other things. The role of microbes in animal health were widely understood and accepted as pathogens in the past, however, more recent research has greatly changed this notion and recognized the importance of microbial life in the functionality of health, immunity, homeostasis, nutrition, and even behavior [3-7]. Early attempts to characterize microbial communities relied on culture based techniques which fell short due to the bias known as the "Great Plate Anomaly" [8]. The microbes identified by culturing were not only less numerous but also less diverse than those directly visualized by microscopy. This led to the idea that some microorganisms were simply unable to grow in culture media and that others could not grow in such abundance in a culture as they were in their natural environment. The study of microbiomes was revolutionized by the advent of next generation sequencing (NGS) technologies. Through the use of 16S rRNA amplicon highthroughput sequencing, the majority of microorganisms in a sample can now be identified and differentiated based on their genetic diversity, rather phenotypic information. This has enabled the 
identification of multitudes of microbes which were not previously identified due to the limits of culture based technology. Furthermore, the use of shotgun metagenomics, transcriptomics, metabolomics, and proteomics have significantly enhanced the ability of researchers to identify and classify microorganisms based on their metabolic abilities and functions, shedding light on the relationships between microbes and their host.

It has been demonstrated that the gastrointestinal microbiome (GIM) is the most rich and diverse microbial community of the many site-specific microbiomes found in and on mammalian species [1,3]. Furthermore, the GIM is very unique to not only the host species [9], but also the individual host organism due to a multitude of variables including genetics, diet, health status, biogeographical environment, and exposure to antimicrobials $[1,3,7,9,10]$. This is a highly sensitive system prone to a variety of disturbances which can have a profound impact on host health. This review will focus on this impact specifically in horses, while also determining what can be learned from studies in other animal models. The equine gastrointestinal system is home to a complex community of microorganisms which have complex interactions with one another and their host that play important roles in immunity and metabolic function [11-13]. Interest in the equine GIM is quickly gaining popularity, likely due to the importance of the gastrointestinal system and its microbiota in equine health and disease. As hindgut fermenters, horses rely on digestive processes from their fibrolytic microbial inhabitants to break down the complex plantbased carbohydrates that make up a large portion of their diet, resulting in short-chain fatty acids that can be utilized as a source of energy $[11,14,15]$. Furthermore, gastrointestinal disease is a leading cause of horse mortality worldwide [12]. Therefore, an enhanced understanding of the physiologic and pathological dynamics of the gastrointestinal tract and its' micro-constituents is vital to the maintenance and improvement of horse health. Identifying the microbial inhabitants of 
the equine gastrointestinal tract and their respective ecological roles in this dynamic environment has challenged scientists for many years. Over 40 studies of the equine microbiome have been published to date and nearly half of these studies have been published within the last 3 years. The vast majority of these studies have relied only on $16 \mathrm{~S}$ rRNA amplicon sequencing to characterize the microbial community membership of fecal samples, which serve as a proxy for the GIM. The applicable knowledge gained from these studies is limited to speculation, as the microbial phylogeny and taxonomy identified by $16 \mathrm{~S}$ rRNA surveys does not provide specific information regarding the metabolic pathways and capabilities of the microbes in the sample [16,17]. Additionally, while commonly used in most host associated microbiome studies, fecal samples may not be truly representative of the GIM of horses because their gastrointestinal tract is made up of several different compartments which each have characteristics that are potentially constraining to microbial life and thus host unique microbial populations [12,13,18-20]. Figure 1 illustrates the variety of bacterial phyla present in differing gastrointestinal compartments in the horse, at both the luminal and mucosal layers [21]. Despite such constraints, these studies have greatly enhanced our understanding of the gastrointestinal microbiome's importance to horse health, and the environmental factors that contribute to microbial community development. 


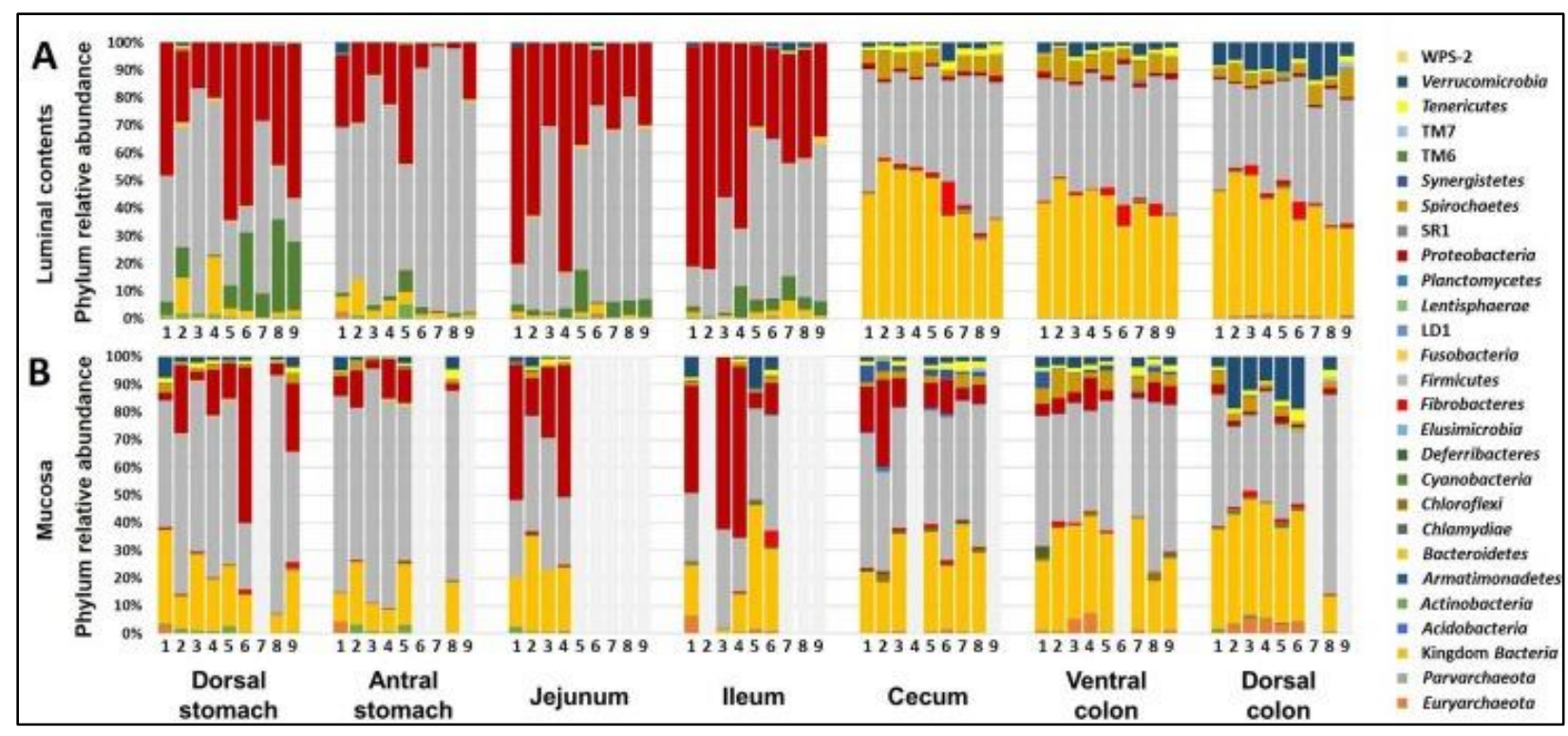

Figure 1. Relative abundance of phyla in luminal and mucosal equine gut microbiota. Bar charts showing relative abundance of phyla detected in luminal contents (A) and mucosa (B) of samples collected from dorsal stomach, antral stomach, jejunum, ileum, cecum, ventral colon, and dorsal colon of nine healthy adult horses, displayed in the same order in each chart and in each GIT region (animal IDs listed below bars). Samples returning fewer than 1000 sequences not shown; legend at right. (Ericsson et al., 2016)

Nutritional influences, such as dietary composition, are a predominant factor of microbiome community membership and stability. Studies in mice and humans have suggested that diet accounts for more variation in GIT microbial diversity than other individual factors such as genetics, geographical distribution, age, gender or body mass index [7,22-25]. As a hindgut fermenter, microbial digestion is an essential step in the breakdown and utilization of feed for horses [26]. In 2016, the idea that the equine gastrointestinal microbiome may be altered to improve digestibility across a variety of diets was reintroduced - in light of the advancements made through next-generation sequencing of the microbiome [27]. The impact of diet related alterations to the equine GIM community may extend past digestive efficiency and could potentially have deleterious health effects associated with an overall shift in the host's homeostasis. An abrupt change from forage-grain diet to strictly pasture diet resulted in a rapid multi-faceted change in the fecal bacterial community structure [28]. Dietary induced alterations to the gut microbiota have 
been demonstrated to result in a significant increase in the frequency of alimentary stress behaviors exhibited by horses [29]. Further research is needed to determine if shifts in the microbial community composition associated with diet have a causative or correlative relationship with diet related gastrointestinal diseases. From an evolutionary stand-point, horses developed their dietary preferences and gastrointestinal functionality based on the intake of large amounts of low quality forage found in the steppe regions near Mongolia, where the ancestors of the domestic horse originated [30]. The evolution of the tooth in the ancestors of modern horses, suggests a shift from a frugivorous diet, to "leafy browsing", ultimately to grass eating or grazing [31]. The most recent ancestors of modern domestic horses were evolved to spend a large portion of their day browsing for forages in the grasslands, and thus maintaining a flow of low-quality nutrients throughout their gastrointestinal tract. Through domestication, horses are subject to habitat constraints as they often live in much smaller pastures and stables and therefore often lose the ability to freely graze throughout the day [32]. To make up for this loss of nutrient availability, and the increase nutrient needs experienced by horses in athletic training or work, domestic horse diets are often supplemented with nutrient concentrated feed stuffs. The transition from a low quality forage based diet to one predominated by a nutrient rich concentrate feed has been associated with the development of a variety of gastrointestinal diseases such as colic, colitis, and laminitis [33]. The role of the microbiota and the prevalence of such diseases has become a major focus of equine GIM research. A recent study was conducted evaluating the temporal stability, functionality and community diversity of the GIM as a response to two diets which differed in nutrient availability [34]. The results suggested that diets consisting of lower nutrient availability resulted in a higher level of diversity and stability over time compared to the higher nutrient availability diet. Another study focused on gastrointestinal disorders associated with the feeding of highly fermentable 
carbohydrate based concentrate diets [35]. The results of this study suggested that the concentrate based diet increased instability of the fecal microbial community when compared to a forage based diet demonstrated by increased variability in the microbiota community composition.

Studies have also demonstrated that the equine GIM composition undergoes significant alterations during disease states. In 2012, one of the first attempts was made to apply NextGeneration Sequencing (NGS) technology to study the equine microbiome [36]. This project compared the fecal microbiome between a cohort of healthy horses versus a cohort of horses with undifferentiated colitis. This study found that in healthy horses, the dominant bacterial phylum was Firmicutes and there were a high prevalence of Clostridia and related organisms in the samples from this cohort. In comparison, samples from the horses with undifferentiated colitis were dominated by the Bacteroidetes phylum and there was also an increased prevalence of Fusobacteria (see figure 2) [36]. It should be noted in the previously described study, that the horses with colitis were all in a hospital setting during the time of sample collection, while the healthy animals came from a variety of different local farms. A project focused on equine grass sickness also utilized NGS to investigate the dysbiosis associated with the onset of this disease. The results of this study suggested that horses with equine grass sickness also had a significant increase in Bacteroidetes and a decrease in Firmicutes, in addition to multiple significant changes in taxonomic abundance at the genus level [37]. Colic is a major concern of horse owners due to its' high prevalence in all breeds and ages of horses. One study observed numerous changes in the fecal microbiome of mares preceding the onset of post-partum colic, which the authors hypothesized, could one day be used to predict and prevent colic episodes [38]. However, in that particular study, the mares that developed post-partum colic $(\mathrm{n}=13)$ experienced 6 different types of colic cases, and significant microbial differences were not described between different colic types. While this study provides 
some suggestive data about changes in the microbiota before colic onset, in order to realistically predict or prevent colic episodes, far more extensive research will be required to investigate the physiological and mechanistic roles that the microbiota may have in specific types of colics. Furthermore, the horses used in this study came from 3 different farms and had access to different diets, but were all given access to pasture after foaling. These variations and changes in diet also could play a role in the onset of colic, and should be accounted for when considering the external variables that can impact the microbiota and gastrointestinal health $[28,38]$.

More recent research has focused on evaluating associations between horse husbandry and the equine GIM. In addition to the biological impact of domestication, the intensive management practices utilized in the horse industry may in some ways be associated with the development of gastrointestinal disease and merit further evaluation [39]. Some researchers investigated the impact of acute exercise and aerobic conditioning on the fecal microbiota, as these activities are regularly experienced by equine athletes as part of a normal training program [40]. The results of this study indicated that both the composition and structure of the fecal microbial community was impacted by exercise. Furthermore, this study found that the use of performance enhancing supplements (chromium or L-carnitine) induced moderate changes in the fecal microbiota of the sample population, but these changes were not significantly different from the horses that did not receive supplementation. Another group has demonstrated that transportation of horses, dietary fasting and the use of anesthesia were all variables that had a significant impact on the fecal microbial community composition and structure of healthy horses [41].

Research has also been conducted which focuses on the management of horse health. For instance, the use of systemic antibiotics has been a common practice in the livestock industry for treating and preventing bacterial infections, although the impact of antibiotic use on the equine 
GIM is not yet fully understood. One study aimed to investigate this by characterizing the changes in the fecal microbiome when a cohort of healthy horses was given different types of antimicrobial drugs. The most profound effects were observed on day 5 of the trial, which was immediately after treatment. By day 30, the microbiota of the fecal samples had returned to a community composition structure more reflective of the baseline samples taken prior to treatment, than the samples taken at earlier time points following treatment. From a taxonomic standpoint, the results of this study suggested that microbial members of the Verrucomicrobia phylum were the most affected by the antimicrobial treatment. The results of this study indicated that the use of systemic antimicrobial drugs leads to changes in the intestinal microbiota but the changes differed based on the specific drug administered [42].

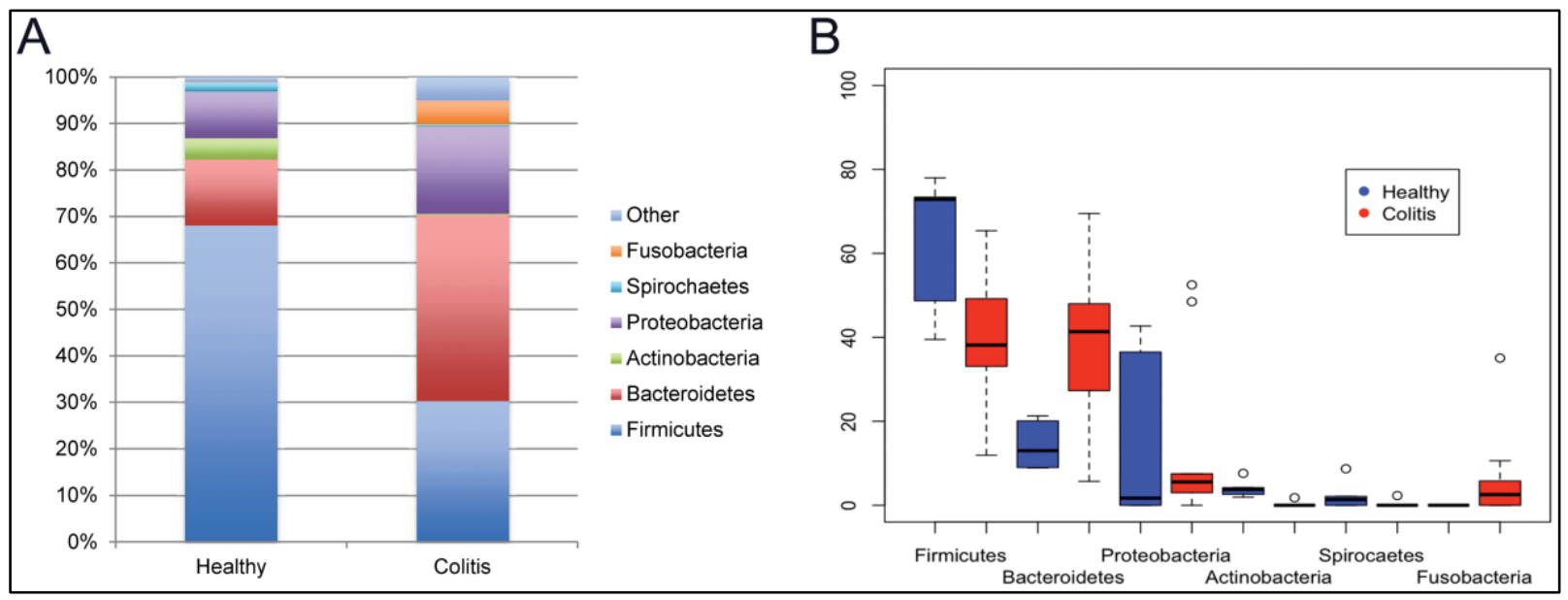

Figure 2. Fecal bacterial population. Overall percentages of bacterial populations at the phylum level (Fig. A) and intra-phylum variation (Fig. B) present in the feces of healthy horses and horses affected by colitis. (Costa et al., 2012)

The equine microbiota is a vital component of horse health, especially the microbial populations specific to the gastrointestinal tract. This dynamic microbial community is very sensitive and quick to respond to changes, which may impact the health of the host horse and vice versa. Research pertaining to the microbiota is quickly evolving and adapting to utilize new technologies that will enable researchers to better understand these microscopic communities. 


\section{Equine Parasitic Helminths}

Domestic horses are regularly exposed to a variety of parasitic helminths that can inhabit and cause infection within the gastrointestinal tract. Internal parasites are known to cause a variety of gastrointestinal disorders in horses [43,44]. Parasitology has been a focus of equine science for many years and the interactions between GI parasites and their horse hosts are well understood [43]. Essentially all horses are host to some population of parasites, especially those with access with pasture or that eat hay directly from the ground, as these activities provide a direct route of helminth egg ingestion [43]. For the most part, the equine host and its' internal parasites are able to coexist without any negative effects at the organism level, although there is pathologic evidence of parasite damage to various organs and tissues $[43,45]$. The majority of helminth infections do not result in clinical signs. A heavy burden of certain parasitic worms, however, can lead to a variety of deleterious physical effects including digestive inefficiency, decreased body condition, ulceration, anemia, recurrent colic, and even fatality without proper intervention. Due to higher stocking densities in domestic horse operations, parasite accumulations are typically much higher compared to natural occurrences in the wild. Therefore, parasite management practices are frequently implemented to avoid high helminth infection levels [43]. The following review will describe the common equine helminths and usage of anthelmintic drugs for parasite control.

\section{Equine Gastrointestinal Parasites}

The Strongylidae order, of the Nematoda phylum, contain two of the most well-known of the equine gastrointestinal parasites: the large strongyles (S. vulgaris, S. edentates, S. equinus) and the small strongyles (40+ species exist and are collectively referred to as cyathostomins). In the past, large strongyles were the most prevalent and pathogenic helminths in horses, although wide- 
spread parasite control practices have greatly reduced their populations, resulting in small strongyles being the more prevalent and concerning of the strongyles today. This shift in strongyle prevalence is further described in this review (see Equine Internal Parasite Control section below). Both large and small strongyles cause the greatest risk of symptomatic disease to their host during their larval life stage. S. vulgaris larvae are known to migrate throughout the branches of the cranial mesenteric artery and can cause parasitic thrombosis and arteritis and result in colic, gangrenous enteritis, intestinal stasis, intussusception or even intestinal rupture [46]. Larvae of the other two large strongyle species, S. edentates and $S$. equinus both migrate to the liver, and $S$. equinus can also migrate to the pancreas [47]. The migration of $S$. edentates and $S$. equinus through hemorrhagic tracts can result in hepatic parenchyma and fibrous scaring. Clinical signs of large strongyle infection include anemia, decreased thriftiness, diminished coat quality, weight loss, poor performance, intestinal rupture, and death [47]. Unlike large strongyles, the small strongyle species do not migrate out of the intestine and their early development is confined to the intestinal walls, where they will eventually emerge from and feed on mucosa and cause damage to the intestinal wall, although this damage is typically less detrimental than that caused by large strongyles [46]. Larval cyathostomiasis is a specific condition caused when the infective cyathostomins larvae become encysted in the lining of the large intestine [48]. Clinical disease resulting from this condition occurs when a large number of these encysted larvae erupt, potentially causing severe damage to the intestinal mucosa, inflammation, hypoproteinemia, colic, weight loss and edema. This condition can be fatal in up to $50 \%$ of cases [49].

Parascaris spp. (Roundworms, Ascarids) are of highest concern in young horses (typically under 2 years of age) and typically do not impact older horses as immunity to this group is acquired with age $[43,50]$. Ascarid eggs are ingested and larvae begin development in the intestinal tract, 
then moving into the liver via the hepatic vein, larvae cause some damage in the liver but due to this organ's regeneration, permanent damage is rarely observed. The larvae then migrate to the lungs where significant and permanent damage can occur. The immune response to larvae in the lungs can result in alveoli damage and scarring. Furthermore, when ascarid larvae reach the trachea they cause irritation that can result in a cough which causes them to be re-swallowed into the gastrointestinal system in their adult stage, where they then can deplete their host of nutrients [46,50-52]. Heavy levels of infection in foals can cause poor growth, ill-thrift, respiratory system inflammation, cough, and nasal discharge [51]. Infection with Parascaris spp. pose a risk of intestinal impact for the host animal due to the large physical size of the parasite. In an animal with a high level of infection, anthelmintic use causing paralysis of the parasites can result in acute small intestinal impaction, as the large volume of paralyzed worms result in a physical blockage within the intestine [43].

A few other helminths impact equids, although not as common and concerning to horse owners as those previously described. Strongyloides westeri are another type of parasite which mainly are found in the small intestine of foals, although health impacts are not common [43]. Anoplocephala perfoliata (tapeworms) are the most prevalent cestodes found in horses, typically at the ileocecal junction, in the cecum and ileum. High levels of infection has been associated with GI inflammation and colic from intussusception [45]. These conditions result from the pathological reaction at the tapeworm attachment site that can be characterized by hyperemia, mucosal thickening and necrotic ulcers [53]. Gasterophilus spp. (horse bots) are the larvae of bot flies, and can infest the stomachs of horses, and physically hook onto the stomach wall causing ulceration and erosions at the attachment site [46]. Pinworms (Oxyuris equi) can be found in the large intestine of horses, but are not known to directly cause any significant impact to 
gastrointestinal health. However, O. equi lay their eggs near the anus which often causes localized irritation leading to scratching that can the result in secondary infections due to broken skin in this area [54]. Horses that are co-housed with ruminants such as cattle may also be exposed to Trichostrongylus axei (hairworms) which can cause gastritis and ulceration, although these helminths are not of significant concern to horse owners [46,55].

\section{Equine Internal Parasite Control}

While parasites have long been recognized as a cause of disease in domesticated horses, the control and treatment of parasite infection have been relatively recent advancements in the health industry. Since their advent in the 1960s, the use of benzimidazole anthelmintic substances for parasite control in horses was widely recommended [56,57]. An anthelmintic is defined as a substance capable of destroying or eliminating parasitic worms [58]. Initially, the use of these substances were based on the recommendation of administration every 8 weeks [56] and were then updated to incorporate rotational use of additional novel anthelmintic drug classes to counteract more types of parasitic helminths throughout their varying life cycles and to limit the potential development of anthelmintic resistance. [43,57,59]. Today, throughout the equine industry, the use of anthelmintic drugs is one of the most common and utilized practices of treating and preventing helminth infections $[43,59]$. It is recommended that horses begin receiving anthelmintic treatments at 2-3 months of age, followed by a minimum of 3 more treatments throughout their first year of life. After this, treatments should be administered on an as-needed basis [59]. Anthelmintic substances which are most commonly used in domestic horses are summarized below (see table 1). 
Table 1. Common anthelmintic drugs used in horses $[43,46,59]$

\begin{tabular}{|c|c|c|c|}
\hline Class & Active Ingredients & Mode of Action & $\begin{array}{l}\text { Parasites } \\
\text { Affected }\end{array}$ \\
\hline Benzimidazoles & $\begin{array}{l}\text { fenbendazole, } \\
\text { oxibendazole, } \\
\text { thiabendazole }\end{array}$ & $\begin{array}{l}\text { Bind to beta tubulin and } \\
\text { prevent microtubule } \\
\text { polymerization }\end{array}$ & $\begin{array}{l}\text { ascarids, } \\
\text { strongyles, } \\
\text { pinworms }\end{array}$ \\
\hline $\begin{array}{l}\text { Tetrahydropyrimidines } \\
\text { (pyrimidines) }\end{array}$ & $\begin{array}{l}\text { pyrantel pamoate, } \\
\text { pyrantel tartrate }\end{array}$ & $\begin{array}{l}\text { Selective acetlycholine } \\
\text { agonists (only effective } \\
\text { against luminal stages) }\end{array}$ & $\begin{array}{l}\text { ascarids, } \\
\text { strongyles, } \\
\text { pinworms }\end{array}$ \\
\hline $\begin{array}{l}\text { Heterocyclic } \\
\text { Compounds* } \\
\text { (piperazines) }\end{array}$ & piperzine* & $\begin{array}{l}\text { GABA } \\
\text { agonists }\end{array}$ & roundworms \\
\hline $\begin{array}{l}\text { Macrocyclic Lactones } \\
\text { (avermectins) }\end{array}$ & $\begin{array}{l}\text { ivermectin, } \\
\text { Moxidectin, }\end{array}$ & $\begin{array}{l}\text { Increase chloride ion } \\
\text { permeability; } \mathrm{GluCl}^{-} \\
\text {potentiators }\end{array}$ & $\begin{array}{l}\text { bots, ascarids, } \\
\text { strongyles, } \\
\text { pinworms }\end{array}$ \\
\hline Isoquinoline-Pyrozines & praziquantel $^{* *}$ & $\begin{array}{ll}\text { Enhances } & \mathrm{Ca}^{++} \\
\text {permeability } & \end{array}$ & tapeworms \\
\hline \multicolumn{4}{|c|}{$\begin{array}{l}\text { *Piperzine used infrequently in horses, no formulation currently approved for equine usage in the US. One product } \\
\text { approved in Canada. }\end{array}$} \\
\hline
\end{tabular}

Current parasite control guidelines and strategies are focused on targeting the most prevalent and most potentially pathogenic parasites [59]. The goal of these guidelines have recently undergone a major shift as they were originally developed when large strongyles (Strongylus vulgaris) were the most important parasite affecting horses [56]. The original guidelines were very successful in controlling S. vulgaris, and their prevalence and infection rate in the equine population has now rapidly decreased. The most recent recommendations developed by the American Association of Equine Practitioners' Parasite Control Subcommittee, focus on 
the control of small strongyles and tapeworms in adult horses and Parascaris spp. in young horses.

These updated recommendations also address the issue of anthelmintic resistance.

The AAEP guidelines utilize the following definition of anthelmintic resistance as published by N.C. Sangster in 1999:

"Resistance is the ability of worms in a population to survive treatments that are generally effective against the same species and stage of infection. Anthelmintic resistance is an inherited trait. The development of resistance first requires that resistance genes are present. The rate of development of resistance is determined by selection pressure and the extent to which worms surviving treatment pass their genes on to the next generation. With continued selection and reproduction of resistant worms, the frequency of resistance genes in the local worm population increases to the point where treatment fails. Once resistance is present, the population of resistant parasites do not appear to revert to susceptibility, so the aims of resistance control are to prevent the first steps in the development of resistance and then to delay the accumulation of resistance genes." $[59,60]$

Initially, the use of rotational anthelmintic treatment was recommended due to the lack of broad-spectrum anthelmintics in addition to the varying seasonal life cycles and infection risk status of helminth species. These recommendations were then continued in an attempt to address the rise of anthelmintic resistance [51]. Due to the history of frequent anthelmintic use targeting S. vulgaris, an unintended selection pressure was exerted on populations of cyathostomins and Parascaris spp. resulting in high levels of anthelmintic drug resistance in these populations [61]. According to the 2015 AHP Equine Industry Survey sponsored by Zoetis, $80 \%$ of participants indicated that parasite drug resistance is a cause for concern among horse owners [62]. Cyathostomins, however, are very mild pathogens and only cause clinical disease in very high infection levels; so, they do require the frequent anthelmintic treatment patterns that increase anthelmintic resistance. Parasite control strategies for adult horses should instead focus on only using specific effective anthelmintic drugs administered on an as-needed basis [43,59]. As previously stated, horses naturally harbor a parasite population without clinical illness effects, therefore the objective of parasite control should be to limit these populations to a level that allows 
the host to stay healthy, rather than attempting to fully eradicate the internal parasite population. Additionally, most anthelmintic drugs are only effective against adult worms, which have passed the larval stage in which they pose the highest risk of disease to their host. Control of adult worms is still essential to limiting the reproduction of, and environmental contamination with, parasite eggs. this emphasizes the need to administer specific dewormer drugs during time periods that are most effective at limiting the shedding of eggs and larval development. In summary, the goals of parasite control programs as outlined by the AAEP guidelines are as follows:

1. To minimize the risk of parasitic disease.

2. To control parasite egg shedding.

3. To maintain efficacious drugs and avoid further development of anthelmintic resistance as much as possible.

To effectively meet these goals, a large effort has been made to inform horse owners about anthelmintic use and encourage the use of parasite infection diagnostic methods prior to anthelmintic administration. The use of fecal egg counts (FECs) is widely accepted and encouraged as a means of monitoring an individual horse's worm burden, egg shedding potential and to monitor the effectiveness of anthelmintic administration [43]. FECs require minimal equipment and training and are readily available through most veterinary practices. More information regarding the use of FECs, recommended protocols, and the interpretation of their results can be found in the full AAEP parasite control guidelines (https://aaep.org/sites/default/files/Guidelines/AAEPParasiteControlGuidelines_0.pdf).

Despite the updated literature and publically available guidelines, horses are frequently dosed with anthelmintic drugs as part of a standardized rotational deworming schedule, without regard to the actual parasitic burden of the individual animal or a medical need for anthelmintic treatment [57]. The 2015 AHP Equine Industry Survey sponsored by Zoetis, reported many informative statistics about the relevance and importance of parasite infection to horse owners, in 
addition to the utilization of anthelmintic drugs and their implementation strategies. The results of this study revealed that the vast majority (88.6\%) of respondents that deworm their horses, choose to do so themselves. According to the survey, $55.3 \%$ of respondents indicated that they stick to a rotational deworming schedule, and nearly half of the respondents deworm their horses four to six times per year. The AAEP has recently recommended that horse owners should base their anthelmintic usage on veterinarian recommendations and the results of regularly conducted fecal egg counts [59]. About $38.2 \%$ of participants in the 2015 AHP survey said that they follow these recommendations by the AAEP and $48 \%$ of respondents said that their veterinarian is involved in the development of deworming strategies for their horse, which is a significant increase from past results. Surprisingly, only $47.5 \%$ of the survey participants reported that their veterinarians recommended the use of fecal egg counts [62]. Interestingly, many countries in Europe have now established policies which limit anthelmintic drug availability to a prescription-only basis and thus require a veterinary approved helminth infection diagnosis via routine FECs [57]. While not universally required, the use of fecal egg counts for helminth infection surveillance are undeniably an important step in the development of parasite control programs. As previously mentioned, some level of helminth infection in horses and other grazing animals is expected and typically does not result in adverse health impacts $[43,63]$. Although, in the U.S. the acceptable level of helminth infection as identified by FECs and the threshold for anthelmintic treatment recommendations remain undecided [59]. Commonly used thresholds for treatment are 200-500 EPG for individual horses [64], however clear universal threshold levels still need to be developed.

In conclusion, the health concerns of equine helminth infections, and management of such infections are a constantly evolving subject that will require continuous research as new problems and solutions continue to develop. Additionally, the potential negative effects of anthelmintic use 
are not limited to the issue of anthelmintic resistance. Anthelmintic treatment has been identified as is a colic risk factor, although the physiological mechanisms associated with this risk are poorly understood in many cases other than impaction colic, which can result from a massive die-off of adult parasites $[43,65]$. A common anthelmintic drug, Moxidectin, has also been associated with a subtle inflammatory response in equine intestinal tissues [66]. Recent investigations have suggested that helminths, and anthelmintics, also directly interact with the gastrointestinal microbiota and can influence the health and performance of their host. The findings of these studies and their potential implications for the field of equine parasitology, and horse health in general, will be further described below.

\section{Microbiota and Helminths}

As previously described, the mammalian gastrointestinal tract is a complex ecosystem consisting of a diverse population of microbial organisms and in some instances, parasitic helminths. The focus of this section is on the presence of, and interactions between, microbes and helminths in the gastrointestinal tract. Over time, these organisms have co-habituated a unique niche environment and co-evolved along with their host $[63,67]$. Studies in human and model organisms have demonstrated a multitude of complex interactions that occur between gut microbes and helminth parasites and the impact of these interactions on hosts' metabolic capabilities and immunological function $[63,67-70]$. In order to better understand the impact of the gastrointestinal ecosystem dynamics on the host, the multidirectional interactions between helminths and microbiota require further investigation. Furthermore, considering the prevalence and economic impact of helminth infection within the livestock industry, in addition to the importance of 
microbiota stability in gastrointestinal health, deciphering the complexities of helminth-microbiota associations are key to improving animal health and productivity $[63,71,72]$.

Studies in human and animal models have demonstrated the effect of parasitic helminth infection on the GIM richness and evenness. Richness is a measure of the number of different species present in a sample or an environment, while evenness refers to the distribution of the different species in the population. Richness and evenness are often considered in their contribution to alpha diversity (see Glossary). These studies have focused on a broad range of model organisms, helminth species and experimental designs, and their varying results reflect this range of variables [63]. For instance, helminth colonization has been associated with an increase in microbiota diversity in terms of species richness and number of observed operational taxonomic units (OTUs) in some studies $[73,74]$. Conversely, other studies have demonstrated a marked decrease in alpha diversity associated with helminth infection [75,76]. These contrasting results may be the due to an observable decrease in alpha diversity associated with gastrointestinal inflammation following helminth infection, followed by an increase or return to baseline in microbial diversity following the initial adjustment period during states of chronic helminth infection [63]. Furthermore, certain parts of the gastrointestinal tract may respond differently to helminth infection. In sheep, infection with Haemonchus contortus resulted in a significant increase in the abundance of bacteria in the abomasum and a decrease in the rumen [77].

Other studies have reported changes in the microbiota composition and structure when the host GI is challenged by helminth infection. In a study using a rat model, animals infected with Hymenolepis diminuta had a significantly different microbial community composition than the control animals, as represented by beta-diversity [78]. Fecal samples from a group of children in Taiwan with pinworm (Enterobius vermicularis) infections revealed many significantly different 
relative abundances of bacteria at the genus level when compared to a similar cohort of children without pinworm infection [79]. Samples collected from a cohort of goats infected with Haemonchus contortus had significantly different abundances of multiple bacterial species compared to a control cohort. Additionally, this study reported that 8 KEGG pathways were predicted to be significantly impacted by infection [80]. These studies demonstrate just a fraction of changes observed in microbiota diversity and composition associated with helminth infection. Further investigations have evaluated the potential functional impact of helminth associated alterations to the gastrointestinal microbiota $[68,69,76,80]$.

Changes in GIM composition associated with helminth infection have the potential to alter the host's ability to extract and utilize nutrients from their diet [68]. The microbiota relies on nutrients from their hosts' diet and in turn increase the metabolic capabilities of their host [3]. Parasites compete for dietary nutrients with microbes, without providing any direct nutritional benefit to the host [76]. In 2012, a study in pigs infected with Trichuris suis revealed a significant reduction in the abundance of Ruminococcus and Fibrobacter following infection which suggested a change in the ability to break down fiber [81]. In addition to changes in relative bacterial abundances, this study focused on changes in bacterial genes associated with metabolism and found a significant reduction in the genes associated with carbohydrate metabolism and the biosynthesis of amino acids following T. suis infection [81]. The results of this study are in agreement with other investigations that have demonstrated changes in specific taxonomic groups and metabolic biomarkers which are associated with the metabolism of fiber, carbohydrates and proteins in animal models challenged with GI helminth infection [63,81-83]. A temporal study which followed microbiota changes in mice during and after Trichuris muris infection, demonstrated that helminth associated changes to the microbiota and subsequent metabolic 
function may be long term and persist once the helminth infection has been cleared [83]. The complex relationships between microbiota alterations, helminth infection, and changes in metabolic capabilities are not yet well defined. Understanding the direction and mechanisms that drive these changes will have important implications for managing the host response to helminth infections $[63,76]$.

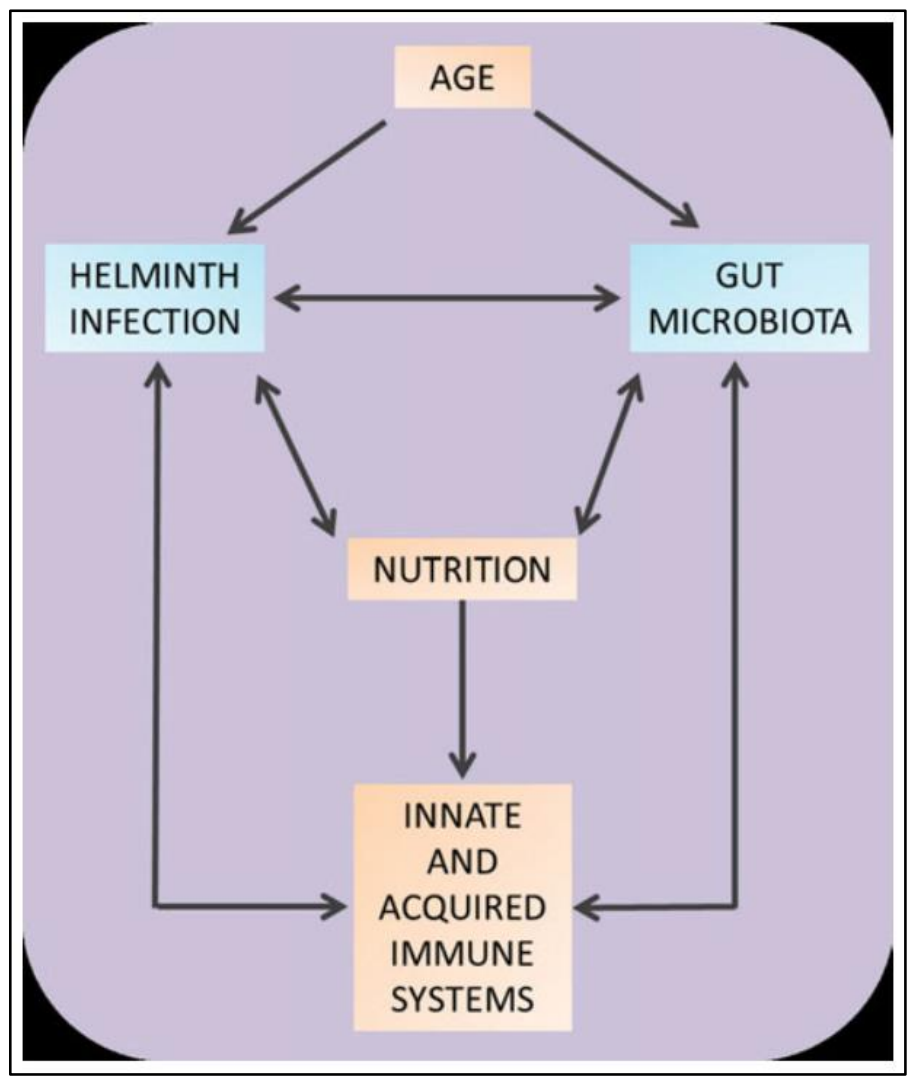

Figure 3. Potential interactions between the gut microbiota and helminth infection (Glendinning et al., 2014)

Interestingly, a review of factors impacting digestive capacities in older horses suggested that the reported reduction in digestion of protein, fiber and phosphorous in geriatric horses may be associated with intestinal damage resulting from a chronic history of $S$. vulgaris infection in the animals studied, as these early investigations occurred before widespread anthelmintic administration [84]. Although this review and previous studies did not evaluate the microbiota's 
role in the observed decreased digestive ability, it would be worthwhile to investigate the impact of chronic helminth infection on the GIM and the long-term effects of digestive efficiency in the host animal.

The intestinal microbiota is vital to the development and function of the mammalian immune system and serves as a barrier against pathogens [2,85]. Interactions between the host immune system and the GIM are driven by the host's necessity to harbor a complex microbiota and to protect the host from potential opportunistic pathogens [4]. Helminths also have many associations with host immunity, with infections promoting immune and regulatory responses that disrupt immune homeostasis [67]. For example, in mice with a schistosome (Schistosoma mansoni) infection, it has been demonstrated that depleting the gut microbiota led to altered schistosome-specific immune responses and a decrease in fecal egg counts; demonstrating the impact of microbiota on the host immune response to helminth infection [86]. Both helminths and microbes must deceive the immune system of their host to some extent to allow their survival, and do so through the activation of suppressive regulatory $T$ cells (Tregs) [67]. This immunomodulation is a key factor in the complexities of the three-way relationship between host, helminth, and microbiota. For instance, administration of the microbe L. taiwanensis in mice increases the frequency of regulatory $\mathrm{T}$ cells and helminth infection establishment rates. This finding demonstrates the promotion of helminth infection due to the presence of commensal bacteria associated regulatory $\mathrm{T}$ cells [85]. An additional study in mice demonstrated that chronic helminth infection resulted in an increase in inflammatory cells, following a significant shift in the balance between Tregs and inflammatory $\mathrm{T}$ cells in the intestinal adaptive immune system [75]. Some of the proposed mechanisms of host immunomodulation associated with the GIM and intestinal helminth infection are summarized in figure 4 below [85]. The physical impact of 
helminth infection including alterations to the intestinal epithelial wall and mucosal production can also alter the environmental conditions necessary for certain microbial species ability to colonize [43,67,81]. Furthermore, the actual establishment of helminth infections may be dependent upon the presence and abundance of microbes within the host gastrointestinal tract [87].

Bacterial microbiota modulation of
helminths

Figure 4. Proposed mechanisms by which intestinal helminths and bacterial microbiota bidirectionally influence persistence in the mammalian host. It has recently become clear that intestinal helminth parasites and members of the bacterial microbiota influence one another's ability to persist in the mammalian intestinal tract. The mechanisms by which they do so are likely multifactorial, site, and context dependent, and likely include direct as well as indirect effects on each other. (Reynolds et al., 2015)

In horses, there has recently been an increased interest in the host-microbiota-helminth interactions. However, helminth-microbiota interactions specific to the equine GIM have not yet been thoroughly investigated, therefore the physiological impact of these potential interactions on the health of the host remains undetermined. The few studies pertaining to the equine microbiota and helminth infection which have been conducted thus far have produced some interesting results. A recent study by Peachy et al. [88] demonstrated that the equine fecal microbial population, investigated via $16 \mathrm{~S}$ rRNA sequencing characterization of the microbiota community composition, was significantly associated with the number of cyathostomin eggs identified via FEC. Samples from horses with lower egg counts had comparatively higher abundances of Methanomicrobia and Dehalobacterium than horses with high egg counts. Other researchers observed differing microbiota and biomarker responses to cyathostomin infection between two cohorts of horses, one 
of which was resistant to parasite infection, and the other was susceptible. This study demonstrated the potential for relationships between the GIM and helminth infection susceptibility and the resulting host immune response [89]. The findings of these studies indicate the need for further horse-specific research pertaining to host-helminth-microbiota relationships. Furthermore, understanding these relationships may provide new perspectives for the field of equine parasitology and research and influence the development of helminth control approaches.

\section{Anthelmintic Use and the Microbiota}

Finally, it is important to tie together the previously described topics and consider the impact of anthelmintic drugs use on the host associated gastrointestinal microbiota. Anthelmintic drugs are widely used in humans and domesticated species to treat helminth infection. As previously described, intestinal parasites are a major point of concern and interest in horses which has driven widespread use of anthelmintic drugs for parasite control. The recent advances and enhanced understanding of the importance of the microbiota, in addition to microbiota-helminth associations, have raised the questions regarding anthelmintic drug use and the potential of previously overlooked implications for the host-associated microbiota. Anthelmintics have the potential to impact the microbiota in a variety of ways; the biological activity of some anthelmintic ingredients may directly inhibit the growth or survival of host-associated microbes and also impact their metabolic process which other members of the microbiota depend upon. Conversely, the successful removal of helminths from the GI tract following anthelmintic administration may result in the removal of helminth associated microbial symbionts. Attempts must be made to differentiate between these potential sources of change when alterations to the host-microbiota are observed following anthelmintic use. 
Multiple studies in humans have demonstrated shifts in the gut microbiota in response to anthelmintic treatment in cohorts of people infected with various intestinal parasitic species $[79,90]$. For instance, a study demonstrated that mebendazole deworming treatment in children infected with Enterobius vermucluaris (pinworms) may have an impact on the microbiota community composition, due to a significant decrease observed in the relative abundance of Fusobacteria and an increase of Actinobacteria [79]. Interestingly, the use of salicylanilide anthelmintics have been proposed as an alternative to antibiotics, due to their bactericidal properties. The antimicrobial mechanisms of these substances are varied and still being investigated, but have been described as inhibiting the enzymes which participate in protein production and cell wall synthesis $[91,92]$. This potential was successfully demonstrated in vitro by inhibiting growth of the common pathogen, Clostridium difficile. Additionally, the anthelmintics used in this study did not appear to significantly hinder the growth of select commensal bacterial species or animal cells [93]. These findings offer an exciting possible solution to address the need for alternative bio-therapeutics due to the rise in antibiotic resistant pathogens [94]. Additionally, this study demonstrates the potential innovation that can result from an improved understanding of helminth-microbiota-host interactions.

Changes in microbiota community composition have also been observed in horses following anthelmintic treatment. Large shifts in the relative abundance of predominating phyla were observed in fecal samples from horses with cyathostomin infection following administration of a combined treatment of Fenbendazole and Ivermectin [95]. In horses shedding a relatively high number of cyathostomin eggs, a significant reduction in the abundance of TM7 and a significant increase in Adlercreutzia was observed following a routine anthelmintic treatment of Ivermectin [88]. A study which evaluated the impact of Moxidectin on the equine microbiome reported that 
the abundance of multiple OTUs differed between treatment groups, although overall bacterial diversity was not significantly altered. This previously described study also evaluated the hindgut fermentation kinetics, and found a significant reduction in fermentation of oats and hay following treatment, but with no significant alterations to bacterial metabolite output observed [96]. Reported changes in microbial community composition associated with anthelmintic administration in horses are preliminary and require further investigation at this time.

\section{Conclusion}

In conclusion, understanding of the importance of the host-associated microbiota is rapidly advancing. Due to the significant number of gastrointestinal health issues in horses, the microbiota associated with this system is an important focus of research at this time. Preliminary evidence demonstrates the need to consider this research in association with parasitology, in regards to interactions between the host associated microbiota, helminths and anthelmintic substances. At this time, the dynamics between these factors which have been characterized in the horse may be subtle, yet are still becoming apparent. As researchers strive to improve our understanding of the gastrointestinal ecosystem dynamics, the unintended consequences of previously accepted medical practices, such as the use of anthelmintic drugs, may come to light. By considering these new perspectives in regards to the importance of microbial life, approaches to animal health management may undergo significant changes in the future. The following chapters describe multiple research projects which were conducted to determine the impact of anthelmintic drug administration on the equine gastrointestinal microbiota. 


\section{CHAPTER II: CHARACTERIZING THE EFFECT OF ANTHELMINTIC ADMINISTRATION ON THE EQUINE FECAL MICROBIOTA IN HORSES WITHOUT AN OBSERVABLE HELMINTH INFECTION}

\section{Introduction}

Disruption of an individual's normal microbiota when the microbial community structure is maladapted or unbalanced, referred to as a dysbiosis, has been associated with a variety of causes and negative effects. A dysbiosis can result when the microbial community composition or structure are altered. Sources of the alteration can be from acute dietary changes, environmental changes, exposure to toxins or pathogens, drugs and stress [97,98]. Dysbiosis is associated with metabolic syndrome [99-101], colitis [36,102] and other gastrointestinal dysfunctions. The possibility exists that routine medical and management approaches - such as treatment with anthelmintic drugs to control parasitic infections - are having more significant impacts on the gastrointestinal microbiota and, by extension, the gastrointestinal physiology. Knowledge of how the microbiota responds to anthelmintic treatment strategies is largely unknown. A recent study by Peachey et al.[103] demonstrated that the GIM community structure differs between individual horses with varying levels of helminth infection and taxonomic abundance differences following anthelmintic treatment were associated with helminth infection level. Given the broad application of anthelmintic drugs regardless of infection status, an important question to consider is whether exposure to a drug intended to kill parasitic helminths have unintended consequences for the microbial community living in the same environment? It is therefore critical we understand the impact of these compounds on the gut microbiota in the absence of any helminth infection. This characterization will help determine the potential for dysbiosis to result from anthelmintic 
treatment. It is important to understand these dynamics for optimal management of equine gastrointestinal health and function.

This study investigates the hypothesis that anthelmintic drugs are capable of altering the equine GIM community composition in animals without an observed cyathostomin or ascarid infection. This hypothesis is assessed through two objectives; 1) characterization of the equine fecal microbiota before and after anthelmintic treatment and, 2) the identification of changes in microbial diversity and taxonomic abundances following anthelmintic administration.

\section{Materials and Methods}

\section{Animal Use}

Animals were housed at the Colorado State University Equine Teaching and Research Center throughout the experiment. All procedures were approved by the Institutional Animal Care and Use Committee (Protocol approval number 16-6811A). Samples were obtained from twelve American Quarter horses (ages 1.5-2 years), housed together in group pens and fed a forage-based diet ad libitum. Animals used had no known history of illness and did not receive any antibiotics in the six months before the study. Fecal egg counts were conducted (see appendix I) on samples collected from all animals prior to the start of this study to confirm that no parasitic helminth (cyathostomins or ascarids) was detectable in any of the animals used. Table 2 includes a summary of the horses used for this study. Two horses (H11 and H12) were excluded from further analysis due to positive FEC results. 
Table 2. Summary of metadata from animals used in this study

\begin{tabular}{|c|c|c|c|c|}
\hline Horse ID & $\mathrm{DOB}^{*}$ & Gender ${ }^{* * *}$ & Breed $^{* * * *}$ & FEC Results \\
\hline H01 & May 2015 & $\mathrm{G}$ & $\mathrm{QH}$ & Negative \\
\hline $\mathrm{H} 02$ & June 2015 & M & $\mathrm{QH}$ & Negative \\
\hline H03 & May 2014 & $\mathrm{G}$ & $\mathrm{QH}$ & Negative \\
\hline H04 & April 2015 & M & $\mathrm{QH}$ & Negative \\
\hline $\mathrm{H} 05$ & April 2015 & M & $\mathrm{QH}$ & Negative \\
\hline H06 & June 2015 & $\mathrm{G}$ & $\mathrm{QH}$ & Negative \\
\hline H07 & March 2015 & $\mathrm{G}$ & $\mathrm{QH}$ & Negative \\
\hline H08 & July 2015 & M & $\mathrm{QH}$ & Negative \\
\hline H09 & April 2014 & $\mathrm{G}$ & $\mathrm{QH}$ & Negative \\
\hline $\mathrm{H} 10$ & May 2014 & $\mathrm{G}$ & $\mathrm{QH}$ & Negative \\
\hline H11 & July 2014 & M & $\mathrm{QH}$ & Positive \\
\hline $\mathrm{H} 12$ & March 2015 & M & $\mathrm{QH}$ & Positive \\
\hline \multicolumn{5}{|c|}{$\begin{array}{l}{ }^{*} \text { DOB: Date of birth } \\
{ }^{* *} \text { Gender: Mare (M) or Gelding }(\mathrm{G}) \\
{ }^{* * *} \text { QH: Registered American Quarter Horse } \\
\text { *****FEC Results: Based on diagnostic fecal egg counts (Appendix I). Positive=egg observed; Negative=No } \\
\text { eggs observed }\end{array}$} \\
\hline
\end{tabular}

\section{Experimental Design and Sample Collection}

Fecal samples were collected at two time points; pre-treatment (day -3) and post-treatment 1 (day 2) resulting in a total of 20 samples (see figure 5). On day 0 , all horses were treated with a single dose $(0.4 \mathrm{mg} / \mathrm{kg}$ body weight $)$ of QUEST® PLUS Gel [active ingredients: Moxidectin and Praziquantel] (Zoetis US) following manufacturer's instructions. Samples were collected immediately following defecation, fecal balls were broken open and $50 \mathrm{ml}$ of material from inside the fecal ball was collected to avoid environmental contaminants. Samples were placed immediately on ice for transport and stored at $-20^{\circ} \mathrm{C}$ freezer within 5 hours of initial collection and remained frozen until DNA extraction. 


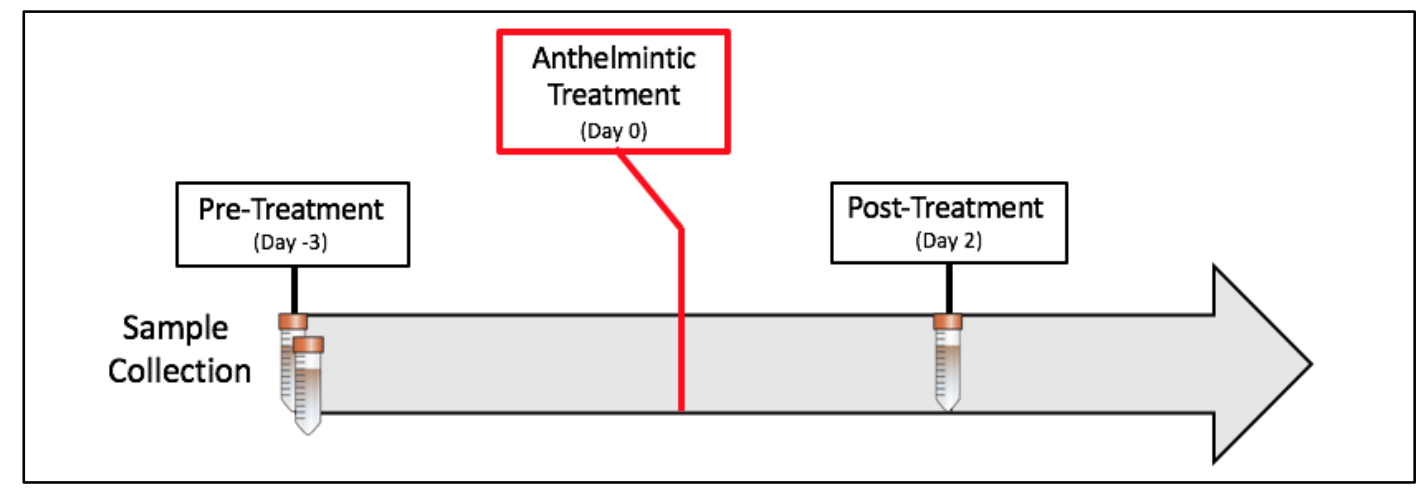

Figure 5. Chapter 2 project experimental design

\section{DNA Isolation and Quantification}

Microbial DNA was isolated from all collected samples according to the protocol described in appendix II. Sample quality control was performed on the isolated DNA samples using 3 methods. A NanoDrop ${ }^{\mathrm{TM}}$ Spectrophotometer (Thermo Fisher Scientific) was used to test the DNA purity and yield (OD260/OD280). An agarose gel electrophoresis test was used to analyze DNA degradation and potential contamination. Then, a Qubit 2.0 fluorometer (Thermo Fisher Scientific) was used to precisely quantify the DNA concentration.

\section{$16 S$ rRNA Amplification and Next-Generation Sequencing}

A 16S rRNA library for the MiSeq Illumina platform was prepared at SeqMatic LLC, USA (Fremont, California) according to the Earth Microbiome Project standard protocol (http://www.earthmicrobiome.org/protocols-and-standards/16s/). A 515f-806r barcoded primer set was used to target the V4 region of the 16s rRNA gene using a dual barcoding protocol. The polymerase chain reactions were performed in triplicate and pooled. Sequencing of the 16S V4 region was performed on the Illumina MiSeq platform (Illumnia Inc., San Diego, CA, USA), using a paired-end 2x150bp cycle run and MiSeq Reagent Kit version 2(300) chemistry. 


\section{Data Processing and Analysis}

Sequence reads were demultiplexed using Illumina's MiSeq Reporter Software (Illumnia Inc., San Diego, CA, USA) and imported into QIIME2 version 2018.2 (https://qiime2.org) for further analysis. The DADA2 pipeline [104] was used to pair forward and reverse reads and for quality control; phiX reads and chimeric sequences were filtered following a pooled consensus method. The resulting feature table was used for taxonomic assignment based on the GreenGenes version 13.8 reference database by training a Naïve Bayes classifier with the QIIME2 q2-featureclassifier plugin [105-109]. De novo multiple sequence alignment was preformed using the MAFFT method [110]. Phylogenetic trees were constructed using the FastTree2 and Midpoint Root methods [111,112].

Absolute count data from the GreenGenes taxonomic assignment was normalized into relative abundances at each taxonomic level and plotted using the QIIME2 taxa plugin. Community alpha and beta diversity were analyzed using phylogenetic and non-phylogenetic metrics through the QIIME2 diversity plugin. Diversity within each sample ( $\alpha$-diversity) was evaluated for evenness and richness based on the Shannon diversity index. The significance of the sample collection time (pre-treatment vs. post-treatment) variable effect on the samples' alpha diversity values was calculated using a Kruskal-Wallis testing method [113]. This method is a nonparametric version of the one-way ANOVA test and corrects for multiple testing using the Benjamini and Hochberg method [114]. Diversity between samples ( $\beta$-diversity) was estimated by calculating weighted UniFrac distances [115] from the feature table. The R software was used to visualize data and generate PCoA plots from distance matrices using the qiime2R package [116,117]. A pairwise PERMANOVA test was applied to determine significant differences between groups of samples, with 999 permutations used to calculate p-values [118]. To analyze 
differences in the microbiome composition before and after anthelmintic treatment, the absolute counts of taxonomic features were tested for differential abundance using the DESeq2 negative binomial algorithm [119]. The Benjamini and Hochberg's method was applied to correct for multiple testing errors [114]. For all comparisons and statistical tests, $\alpha=0.05$ was set as the threshold for significance.

\section{Results}

16S rRNA Amplicon Sequencing Data Summary

Demultiplexed sequences were imported into QIIME2 and sequencing data results were summarized. For the 20 samples, the raw data resulted in a total of 3,475,695 sequences (see table 3).

Table 3. Summary of amplicon sequencing data generation

\begin{tabular}{|l|l|}
\hline \multicolumn{2}{|c|}{ Demultiplexed Sequence Counts Summary } \\
\hline Minimum & 87923 \\
\hline Median & 174899.5 \\
\hline Mean & 173784.75 \\
\hline Maximum & 252304 \\
\hline Total & 3475695 \\
\hline
\end{tabular}

The distribution of the basepair qualities at each position in the sequence reads were visualized (see figure 6). The vast majority of sequences had a quality score above 35 , so the data was not further truncated or trimmed for removal of low quality sequences [120]. 


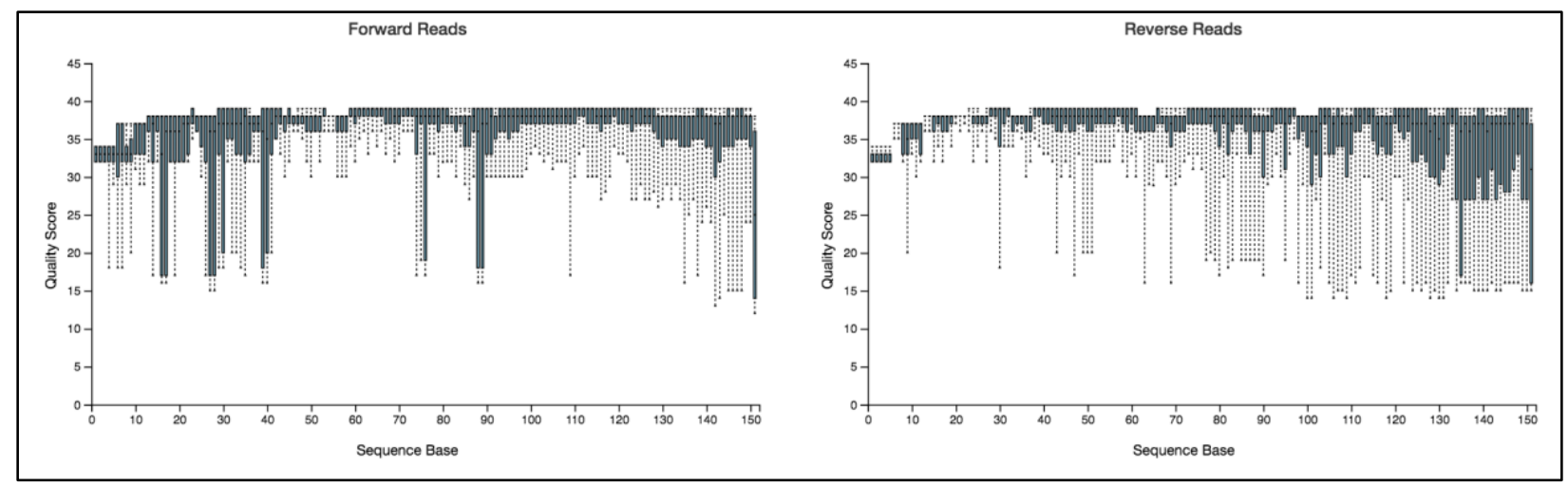

Figure 6. Distribution of sample sequencing quality

Following quality control using the DADA2 pipeline, a feature table was constructed to map feature identifiers to representative sequences. Within the 20 samples, there were 5,369 total features, with a total frequency of 2,640,452. There was a mean frequency of 132,022.6 (range: 75,931.0-220,178.0) total features per sample. For each feature, there was a mean frequency of 491.8 observations (range: 1.0-36,448). For downstream analysis, the frequency tables were rarified at an even sampling depth of 75,931 features per sample. At this level, 1,518,620 of the original sequences (57.51\%) and all of the 20 samples were retained for analysis.

\section{Microbial Community Diversity}

Alpha and beta diversity metrics were determined for the sample microbial communities and the effect of treatment on diversity measures was evaluated. A decreasing trend in $\alpha$-diversity was observed in the Shannon Diversity Index following treatment (see figure 7). The observed effect of sample collection time (pre-treatment vs. post-treatment), was statistically significant (pvalue $=0.0493)$. 


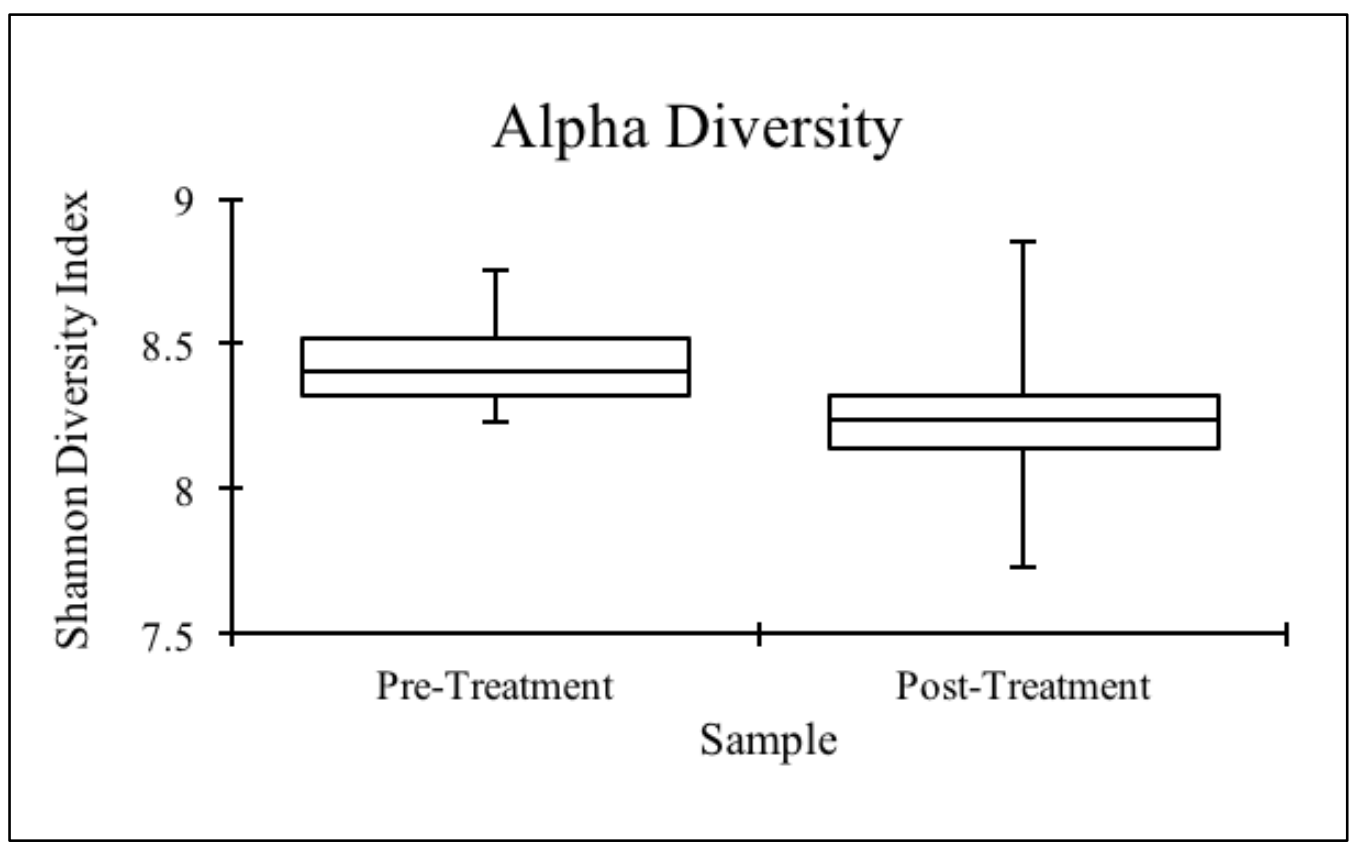

Figure 7. Alpha Diversity distribution boxplots between pre- and post-treatment sample groups

Differences in community diversity between pre and post treatment samples ( $\beta$-diversity), represented by weighted UniFrac distances, was not significant ( $\mathrm{p}$-value=0.2930). There was, however, slight clustering observed by collection time point (see figure 8 ). The effect of individual horse (see figure 9) was highly significant (p-value=0.001). 


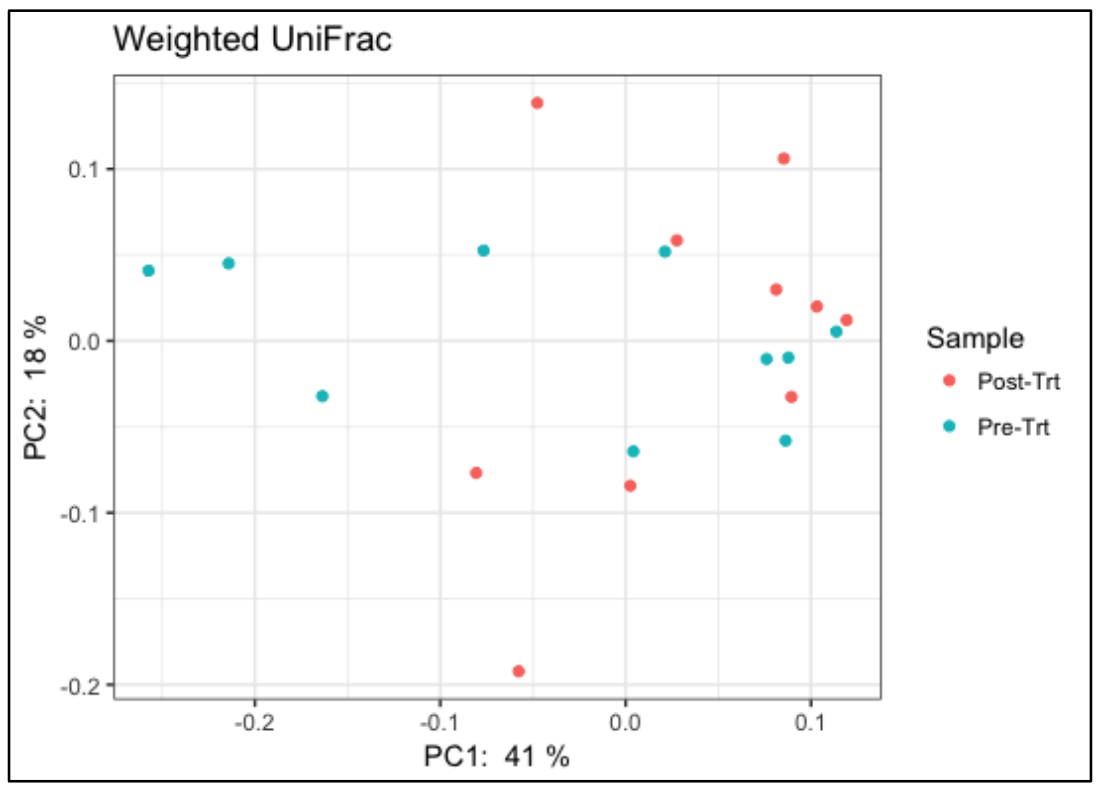

Figure 8. Weighted UniFrac PCoA labeled by sample collection time group

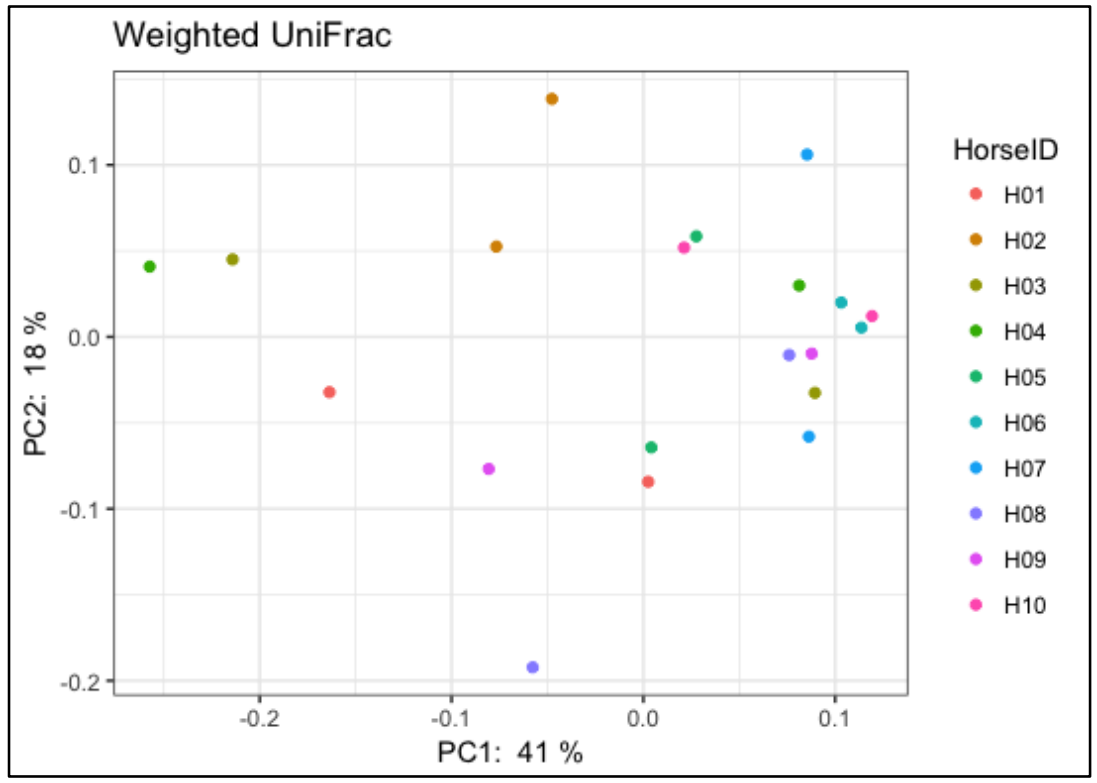

Figure 9. Weighted UniFrac PCoA labeled by individual horse ID

\section{Microbial Community Composition}

Taxonomic community profiles of the samples were pooled and the relative abundance of taxonomic groups were compared between the pre-treatment and post-treatment samples (see 
figure 10). Across both collection time points, the Bacteroidetes phylum predominated the community membership and increased following treatment (pre: $42.4 \%$, post: $45.9 \%$ ). The Firmicutes were the second most abundant phylum in all of the samples and decreased after treatment (pre: $25.0 \%$, post: $21.4 \%$ ). Other notably abundant phyla include the Spirochaetes (pre: 13.3\%, post: $14.9 \%$ ), Verrucomicrobia (pre: $9.6 \%$, post: $8.8 \%$ ), and Fibrobacteres (pre: $4.1 \%$; post: 3.6\%). At the family level, unassigned members of the Bacteroidales order accounted for the majority of community membership at both sampling time points (pre: $23.1 \%$, post: $25.1 \%$ ). Other predominant bacterial families include Spirochaetaceae (pre: 13.3\%, post: 14.8\%), Paraprevotellaceae (pre: 10.6\%, post: $12.9 \%$ ), Ruminococcaceae (pre: $10.3 \%$, post: $7.8 \%$ ), and RFP12 (pre: $8.4 \%$, post: $7.9 \%$ ). At the genus level, the microbial community was again predominated by unassigned members of the Bacteroidales order (pre: $21.0 \%$, post: $23.1 \%$ ). Treponema was the second most relatively abundant genus at both sampling time points (pre: 13.2\%, post: $14.8 \%$ ). Other notably abundant genera include: Fibrobacter (pre: $4.1 \%$, post: $3.6 \%$ ), YRC22 (pre: $3.6 \%$, post: $5.4 \%$ ), Prevotella (pre: $3.4 \%$, post: 3.5\%), Phascolarctobacterium (pre: 2.9\%, post: $3.1 \%$ ), CF231 (pre: $2.9 \%$, post: $3.1 \%$ ), and Oscillospira (pre: $1.9 \%$, post: $0.8 \%$ ). Additionally, three more of the top ten most abundant genera include an unassigned member of the RFP12 family (pre: $8.4 \%$, post: $8.0 \%$ ), an unassigned member of the Ruminococcaceae family (pre: $6.3 \%$, post: $4.5 \%$ ) and an unassigned member of the Lachnospiraceae family (pre: $3.8 \%$, post: $4.5 \%$ ). 


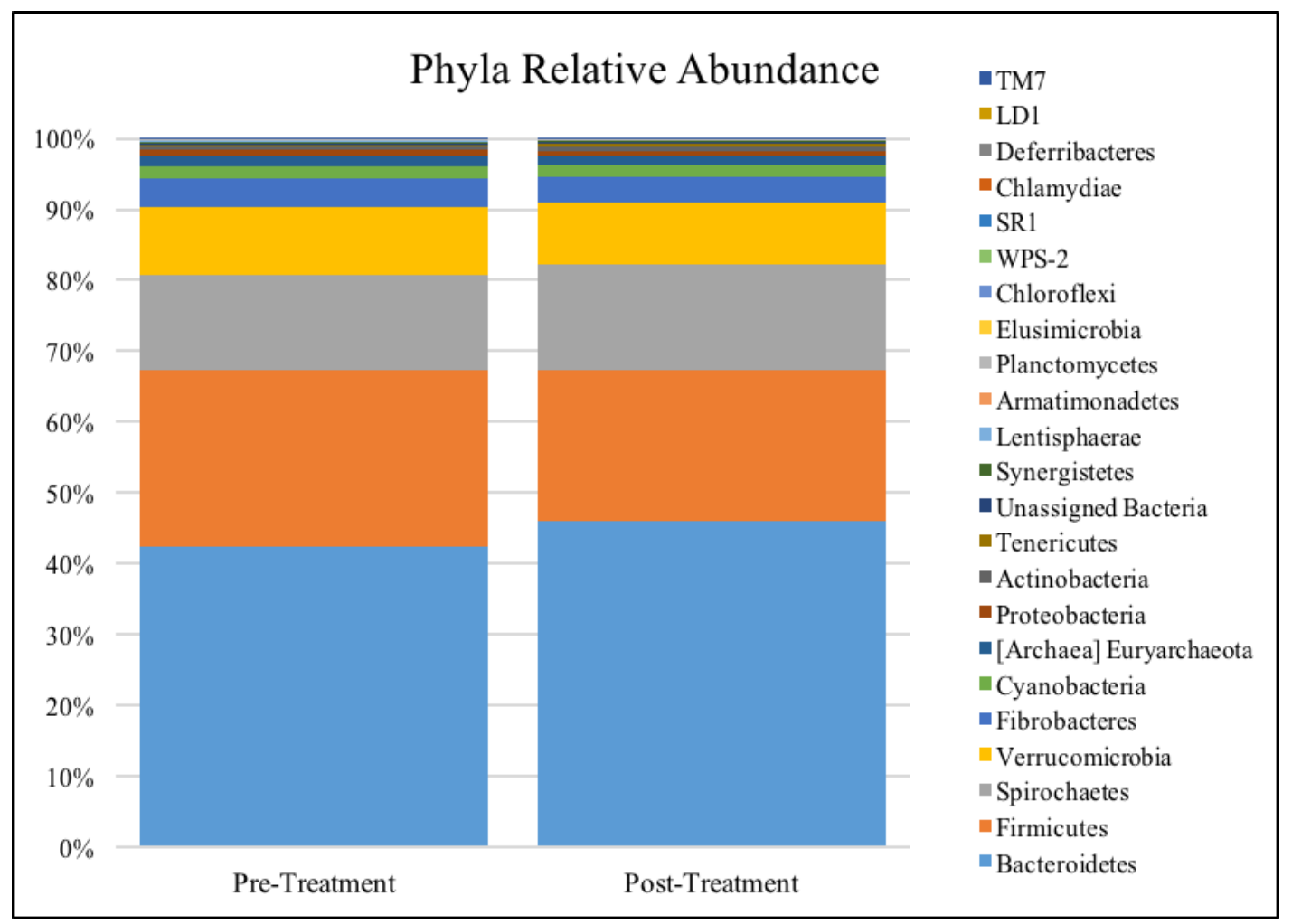

Figure 10. Observed relative abundance of phyla in samples grouped by sample collection time

\section{Differential Abundance of Taxonomic Features Between Sample Collection Groups}

Differential abundance between the community composition of samples before and after anthelmintic treatment was evaluated using the DESeq2 method. Twenty-one taxonomic features were significantly (padj-value<0.05) different between the pre-treatment and post-treatment sample groups. Of these, 7 features from the Bacteroidetes (5) and Firmicutes (2) phyla were significantly higher in abundance in the pre-treatment group. Higher abundance Bacteroidetes include: 3 unassigned members of the Bacteroidales order, an unassigned member of the BS11 family, and YRC22 spp. Higher abundance Firmicutes include: an unassigned member of the Ruminococcaceae family and a Phascolarctobacterium spp. Fourteen taxonomic features were significantly lower in abundance in the pre-treatment group, representing members of the Firmicutes (4), Bacteroidetes (6), Verrucomicrobia (2), Spirochaetes (1) and Cyanobacteria (1) 
phyla. Lower abundance Firmicutes include: an unassigned member of the Clostridiales order, an unassigned member of the Christensenellaceae family, an unassigned member of the Ruminococcaceae family, and Streptococcus luteciae. Lower abundance Bacteroidetes include 2 unassigned members of the Bacteroidales order, 2 unassigned members of the RF16 family, CF231 spp., and Prevotella spp. Additionally, there were lower abundances in 2 unassigned members of the RFP12 family of the Verrucomicrobia phylum, a Treponema spp. of the Spirochaetes phylum, and an unassigned member of the YS2 order within the Cyanobacteria phylum. 


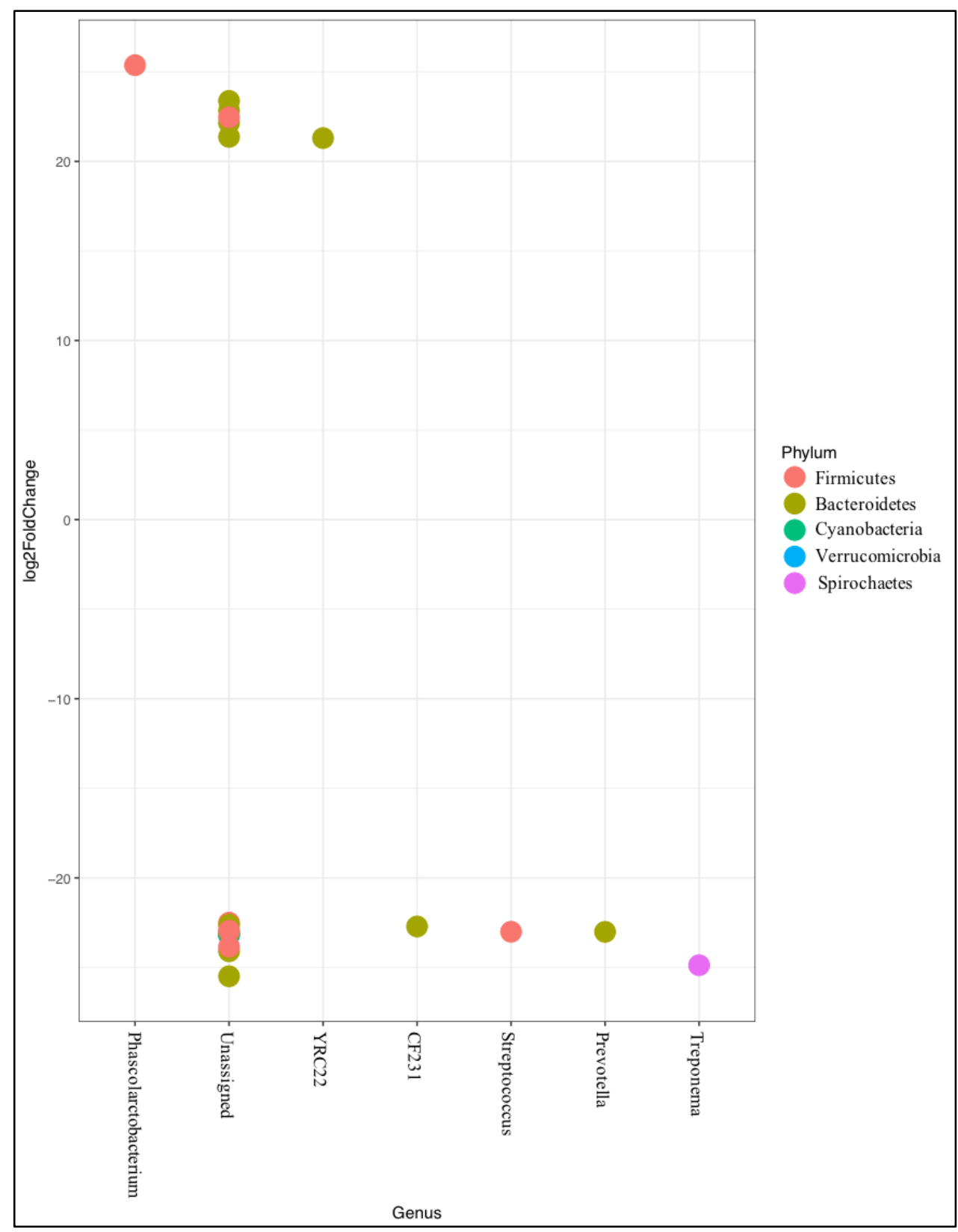

Figure 11. DESeq2 analysis results. Taxonomic features which decreased in abundance following treatment are represented by a positive log2fold change, increased features have a negative $\log 2$ fold change.

\section{Discussion}

Helminth infections are common in the horse, as are the use of anthelmintic drugs to limit and control these infections. Previous studies have demonstrated that changes occur to the 
microbiota in horses with helminth infection following anthelmintic treatment $[95,96,121]$. Due the to the prevalence of anthelmintic use in un-infected horses [43], it is important to determine if anthelmintics impact the microbiota of healthy horses as well. Furthermore, the impact of helminth infection on the microbiota composition and structure may limit the ability to differentiate between the drivers of change in this complex system; as microbial changes could be host-associated or helminth-associated [63]. This is the first study to characterize the equine fecal microbiome before and after anthelmintic administration in a cohort of horses without an observed cyathostomin infection. Differences in the diversity and the taxonomic profiles of samples collected pretreatment and post-treatment suggest that anthelmintic administration may have some impact on the equine gastrointestinal microbiota in the absence of helminth infection. Alterations to the microbial community composition and structure are often referred to as "dysbiosis". It has been hypothesized that dysbiosis can be categorized in three types; (1) loss of beneficial microbial organisms, (2) expansion of pathobionts or potentially harmful microorganisms and (3) loss of overall microbial diversity [122]. Trends in our data suggest that anthelmintic administration may induce dysbiosis, demonstrated by a loss of overall diversity and change in community structure. At this time, it is unknown whether these changes translate into health consequences and remains to be studied.

Alterations in alpha diversity metrics can be accounted for by changes in the number and/or distribution of taxonomic features in a community. A reduction in alpha diversity has been associated with a variety of disease states $[123,124]$, often due to the reduction of certain taxa and the subsequent increase of others, particularly in the gut. The significant decrease in $\alpha$-diversity detected following treatment suggests dysbiosis-inflicting alterations in the community membership and taxonomic abundances may be associated with anthelmintic administration. 
While changes in diversity serve as a broad indicator of community alterations, the mechanisms driving these changes require further investigation to understand the potential impact of dysbiosis [125]. In this study, changes identified in the abundance of certain taxa following treatment explain the observed reduction in $\alpha$-diversity and are consistent with the findings of previous studies $[95,96,121]$. These specific taxonomic changes provide additional support for the notion that anthelmintic-induced microbiota changes could lead to a dysbiosis as previously defined.

Our data suggests that anthelmintic administration may impact taxonomic groups which are functionally important to the health, immunity and metabolic capabilities of horses. Five features belonging to the Clostridiales order of the Firmicutes phylum were identified as differentially abundant between pre- and post-treatment sample groups. This taxonomic group contains many commensal bacteria species that play vital roles in gastrointestinal homeostasis [6]. In horses, members of this taxonomic group predominates the hindgut $[15,126]$ and notable changes in their abundance have been associated with gastrointestinal health and disease [36,127].

The results of this study indicate that microorganisms associated with metabolic processes may be impacted by anthelmintic treatment. For instance, an unassigned Phascolarctobacterium spp. was significantly decreased following treatment. This genus has been isolated from gastrointestinal samples in many mammal species, including the equine cecum and feces $[128,129]$, and is hypothesized to utilize succinate generated from other bacterial species cohabitating the gastrointestinal tract, thus contributing to the metabolic functionality of the microbial community as a whole [130]. A decrease was also observed in members of the Ruminococcaceae family, which are associated with the degradation of cellulose and hemicellulose in the mammalian gastrointestinal tract [131,132]. 
Different compartments of the equine gastrointestinal tract serve unique roles in digestion and metabolism, and thus host specialized bacterial populations $[15,126]$. The cecum is an important location for microbial digestion in the equine large intestine [13]. In this study, changes were observed in multiple taxonomic groups which have previously been identified in high abundance in the equine cecum including Phascolatctobacterium spp., Streptococcus luteciae, and members of the RFP12, RF16 and BS11 families [15,126,128-130,133,134]. These findings suggest that anthelmintic drugs may be particularly active in the equine cecum, and thus impact the metabolic efficiency within this compartment. Compartmental variation is not necessarily represented in fecal samples, so these findings are only speculative and based on the previously documented presence of these species in the cecum $[13,18]$. Due to the importance of microbial fermentation for equine metabolic efficiency, maintaining homeostasis throughout the gastrointestinal tract is crucial to ensuring that animals are able to meet their nutritional needs and avoid metabolic-associated disorders $[14,15,135]$. Furthermore, the taxonomic changes observed in this data may help explain the findings from a recent study, which indicated that anthelmintic administration reduces hindgut fermentation kinetics [96]. Additional research including metabolomics and measures of feed efficiency would be needed to further determine the impact of anthelmintics on equine metabolic capabilities.

Interestingly, the results of the differential abundance analysis indicated the expansion of multiple taxonomic groups that have previously been identified as potential pathogens. A notable example of this was the increase of Prevotella spp. observed following anthelmintic administration. This genus has members that can be both commensal and pathogenic in nature [136-139]. In horses, Prevotella spp. have been described as opportunistic pathogens associated with a variety of diseases including periodontitis, lower respiratory tract infections, and colitis 
$[36,140,141]$. Research into the pathogenesis of Prevotella spp. has been more thorough in human and mouse models, demonstrating many compelling associations between Prevotella spp. abundance and inflammatory diseases [137]. Furthermore, the abundance of Streptococcus luteciae was significantly increased in the post-treatment sample group. This species has been observed to predominate the equine hindgut prior to the onset of laminitis [142]. An increase in $S$. luteciae has also been observed during the onset of colorectal carcinogenesis associated with chronic colitis, suggesting that this species may act as an opportunistic pathogen [143]. Additionally, a taxonomic feature from the Treponema genus also increased in abundance following treatment. Species from the Treponema genus have been previously identified in equine microbiome samples from both healthy and disease compromised animals [144-146]. A previous study has suggested that Treponema spp. may be associated with hoof canker disease pathogenesis [144]. This genus has also been isolated in equine oral samples and is associated with periodontal disease [147]. It should be noted that the above mentioned observations have not been associated with disease causation, but their role in equine gastrointestinal dysbiosis may warrant further investigation.

The results of the $\beta$-diversity analysis suggest that differences in the microbial composition were more pronounced between different individual animals, than between different sampling time points. This notable result was not entirely surprising, as studies in human and mouse models have suggested that the inter-individual effect is a major driver of variation in microbial community structure and composition [148-151]. The highly significant individual effect observed in this data suggests a need for a larger sample size to improve the ability to detect biological trends in between sample variation due to treatment. The animals used in this study were all of the same breed with a relatively small distribution of age and gender. Additionally, all of the horses used were fed the 
same diet, lived in group housing, and had no known previous diagnosis of infection or disease. The standardization of these potentially confounding variables [135] should limit the individual variation resulting from environmental conditions.

\section{Limitations}

Recent efforts have begun to characterize the microbiome associated with helminth infections in horses and other model organisms $[63,121,152,153]$. Additional studies are needed to improve our understanding of the associations between the parasites and microbes cohabitating a hosts' gastrointestinal tract, and how such associations impact the host itself. Determining such associations are also necessary to confirm and specify the impact of anthelmintic administration on the gastrointestinal microbiome in regards to parasitic infection status. The results of this study suggest that the combined administration of Moxidectin and Praziquantel does have an impact on the equine fecal microbiome composition. Future investigations will also need to address the broad range of anthelmintic drugs currently available, as their varied mode of actions may differentially impact the gastrointestinal microbiome. Additionally, the scope of this study was limited to a single post-treatment sample collection time point; additional time points at smaller and larger time intervals following treatment may be useful in characterizing both the short term and long term impacts of anthelmintic use.

The Bacteroidetes was the predominate $(44.2 \%)$ phylum identified in all samples in this study, followed by the Firmicutes (23.2\%), Spirochaetes (14.1\%), Verrucomicrobia (9.2\%), and Fibrobacteres $(3.8 \%)$. The predominating phyla observed in these samples are consistent with other studies in animals with a forage-based diet $[9,121,154]$, although some studies have shown Firmicutes to be the predominant phylum in equine fecal samples $[12,36,89,127,155]$. These 
inconsistencies could be due to many factors including differing DNA extraction protocols, $16 \mathrm{~S}$ rRNA amplicon target regions, sequencing depth and platforms, in addition to the animal selection criteria used for each study [12,135].

Finally, the interpretation of these findings, along with many marker gene microbiome studies in horses and other less-studied model organisms, is limited by the lack of taxonomic resolution provided by microbial reference genome databases. In order to better understand the functionality, and potential implications of the host-associated microbiota on a broad scale, it is essential that the individual microbial organisms that makeup the microbiota are thoroughly studied and described. The ability to identify microbes at the species and strain level, and to understand their functional roles in the gastrointestinal ecosystem, will greatly enhance our ability to interpret biological trends observed in microbiota studies.

\section{Conclusion}

The fecal microbiota was characterized before and after anthelmintic administration in a cohort of horses that were not detectably shedding helminth eggs at the time of treatment administration. The findings of this investigation suggests that administering anthelmintic drugs to horses in the absence of an observed helminth infection results in subtle gastrointestinal dysbiosis, indicated by a decrease in microbial diversity and significant changes to the microbiota community composition and structure. Due to the high prevalence of anthelmintic drug usage in horses worldwide, it is pertinent to understand the physiological impact and potential health risk factors associated with the administration of such drugs. The effects observed in this pilot study are preliminary in nature and warrant further investigations to determine how these effect may impact host health. 


\section{CHAPTER III: THE EQUINE FECAL MICROBIOTA ASSOCIATED WITH HELMINTH EGG SHEDDING LEVELS AND THE TEMPORAL IMPACT OF ANTHELMINTIC ADMINISTRATION}

\section{Introduction}

The equine gastrointestinal tract is a complex ecosystem which harbors both microbial organisms and parasitic worms (helminths). Members of this ecosystem play many roles in the health and homeostasis of their host organism. To further understand the impact of the gastrointestinal inhabitants on their host, it is vital to first understand the dynamic relationships occurring between members of the ecosystem. The first objective of this study is to characterize the microbiota community composition and structure in cohorts of horses with varying levels of helminth infection statuses. Furthermore, this study aims to follow-up with the pilot study presented in the previous chapter, by determining the impact of anthelmintic drug administration on the equine microbiota. To address this aim, the current study has two additional objectives: 1) characterize the daily temporal variation observed in the equine microbiota community following anthelmintic drug administration, and if possible, 2) determine if changes to the microbiota following anthelmintic administration vary based on the level of helminth infection detected prior to treatment.

\section{Materials and Methods}

\section{Animal Use}

Twenty-five horses were used in this study. All horses used were American Quarter horses (aged 1.5-2 years at time of study), house together in group pens and fed a forage based diet ad libitum (further metadata summarized in table 4). Criteria for including animals in the study 
included; no known history of illness and no administration of antibiotics, anthelmintics or other medications in the 90 days prior to the study. All procedures were approved by the Institutional Animal Care and Use Committee at Colorado State University (Protocol approval number 166811A).

Table 4. Summary of metadata from animals used in this study

\begin{tabular}{|c|c|c|c|c|c|}
\hline Horse ID & DOB $^{*}$ & Gender ${ }^{* *}$ & Breed $^{* * * * 4}$ & $\begin{array}{l}\text { Strongyle } \\
\text { FEC }\end{array}$ & $\begin{array}{l}\text { Ascarid } \\
\text { FEC }\end{array}$ \\
\hline H01 & May 2016 & M & $\mathrm{QH}$ & 0 & 0 \\
\hline $\mathrm{H} 02$ & May 2016 & M & $\mathrm{QH}$ & 100 & 0 \\
\hline H03 & June 2016 & M & $\mathrm{QH}$ & 25 & 0 \\
\hline H04 & May 2016 & M & $\mathrm{QH}$ & 725 & 0 \\
\hline H05 & June 2016 & G & $\mathrm{QH}$ & 75 & 0 \\
\hline H06 & March 2016 & M & $\mathrm{QH}$ & 775 & 0 \\
\hline H07 & NA & M & $\mathrm{QH}$ & 625 & 0 \\
\hline H08 & NA & M & $\mathrm{QH}$ & 400 & 0 \\
\hline H09 & April 2016 & M & $\mathrm{QH}$ & 0 & 0 \\
\hline H10 & March 2016 & M & $\mathrm{QH}$ & 925 & 0 \\
\hline H12 & May 2016 & G & $\mathrm{QH}$ & 175 & 0 \\
\hline H14 & May 2016 & G & $\mathrm{QH}$ & 900 & 150 \\
\hline H15 & May 2016 & $\mathrm{G}$ & $\mathrm{QH}$ & 300 & 0 \\
\hline H17 & April 2015 & M & $\mathrm{QH}$ & 75 & 0 \\
\hline H19 & May 2015 & M & $\mathrm{QH}$ & 25 & 0 \\
\hline $\mathrm{H} 20$ & May 2015 & M & $\mathrm{QH}$ & 125 & 0 \\
\hline $\mathrm{H} 21$ & March 2016 & G & $\mathrm{QH}$ & 125 & 0 \\
\hline $\mathrm{H} 22$ & March 2016 & $\mathrm{G}$ & $\mathrm{QH}$ & 100 & 0 \\
\hline $\mathrm{H} 23$ & March 2016 & $\mathrm{G}$ & $\mathrm{QH}$ & 750 & 0 \\
\hline $\mathrm{H} 24$ & April 2016 & M & $\mathrm{QH}$ & 425 & 0 \\
\hline $\mathrm{H} 25$ & March 2016 & M & $\mathrm{QH}$ & 325 & 0 \\
\hline $\mathrm{H} 27$ & NA & M & $\mathrm{QH}$ & 1700 & 0 \\
\hline $\mathrm{H} 28$ & NA & M & $\mathrm{QH}$ & 875 & 75 \\
\hline $\mathrm{H} 29$ & NA & M & $\mathrm{QH}$ & 1200 & 0 \\
\hline $\mathrm{H} 30$ & NA & M & $\mathrm{QH}$ & 2250 & 50 \\
\hline
\end{tabular}




\section{Experimental Design and Sample Collection}

Fecal samples were collected at 24 hour intervals over 4 days; day 0 , day 1, day 2 and day 3 (figure 12). Following D0 sample collection, all horses were administered a single dose of QUEST® PLUS Gel [active ingredients: Moxidectin and Praziquantel] (Zoetis US) at the manufacturer's recommended dose of $0.4 \mathrm{mg}$ Moxidectin $/ \mathrm{kg}$ body weight. Samples were collected immediately after defecation, fecal balls were broken open and $50 \mathrm{ml}$ of material from inside the fecal ball was collected to avoid environmental contaminants. Samples were immediately placed in a cooler on ice $\left(4^{\circ} \mathrm{C}\right)$ for transit. Samples were stored in a $-20^{\circ} \mathrm{C}$ freezer within 5 hours of initial collection and remained frozen until DNA extraction. Aliquots from all samples collected were used for bacterial DNA isolation as described in appendix II.

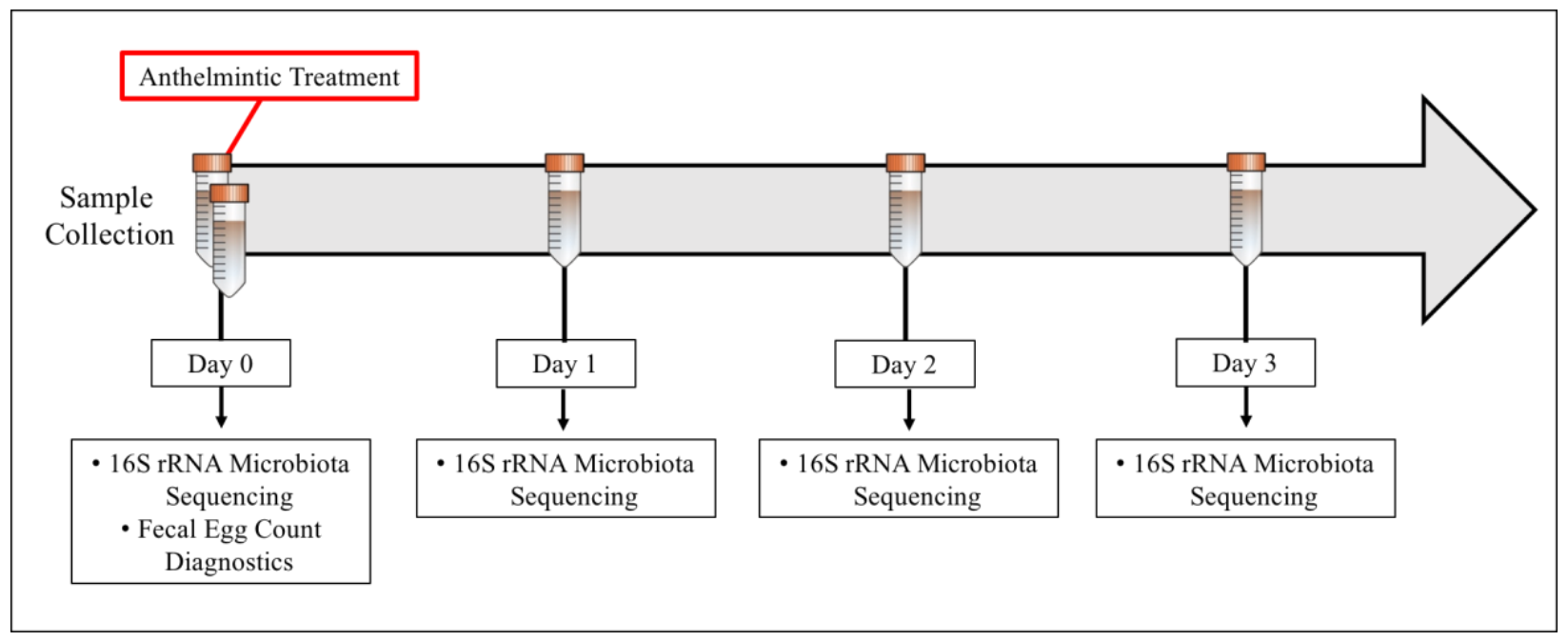

Figure 12. Chapter 3 project experimental design and sampling scheme

Additional samples collected on day 0 from each animal were used for fecal egg count parasite infection diagnostics described in appendix I. Animals were grouped into each of the following helminth egg shedding groups established from the identification of strongyle eggs resulting from FEC diagnostics (table 5): low egg shedder (0-200; LS), moderate egg shedder 
(200-500; MS) or high shedder (>500; HS). The strongyle shedding level group classifications used in this study are based on guidelines suggested by previous research [57].

Table 5. Suggested guidelines for classifying horses into different levels of strongyle egg shedding and the expected percentage of the horse population belonging to each group (Kaplan and Nielsen, 2010; Adapted from the AAEP Parasite Control Guidelines).

\begin{tabular}{|l|l|}
\hline \multicolumn{2}{|c|}{ Egg Count Level } \\
\hline Low Shedders (LS): & $0-200 \mathrm{EPG}$ \\
\hline Moderate Shedders (MS): & $200-500 \mathrm{EPG}$ \\
\hline High Shedders (HS): & $>500 \mathrm{EPG}$ \\
\hline
\end{tabular}

\section{DNA Isolation and Quantification}

Microbial DNA was isolated from all collected samples according to the protocol described in appendix II. Additionally, a "blank" sample, containing only nuclease-free water was included to provide a negative control for DNA isolation, PCR amplicon generation and sequencing protocols. The purity and quantification of isolated DNA were assessed using the NanoDrop ${ }^{\mathrm{TM}}$ Spectrophotometer (Thermo Fisher Scientific) was used to test the DNA purity and yield (OD260/OD280).

\section{S rRNA Amplification and Next Generation Sequencing}

From the isolated genomic DNA, the V4 hypervariable region of the 16S rRNA gene was PCR-amplified using a 515F/806R universal primer set (Forward: 5'-GTG CCA GCM GCC GCG GTA A-3'; Reverse: 5'-GGA CTA CHV GGG TWT CTA AT-3'). All PCR reactions were carried out in $30 \mu \mathrm{L}$ reactions with $15 \mu \mathrm{L}$ of Phusion ${ }^{\circledR}$ High-Fidelity PCR Master Mix (New England Biolabs); $0.2 \mu \mathrm{M}$ of forward and reverse primers, and about $10 \mathrm{ng}$ of template DNA. Thermal 
cycling consisted of initial denaturation at $98{ }^{\circ} \mathrm{C}$ for 1 minute, followed by 30 cycles of denaturation at $98{ }^{\circ} \mathrm{C}$ for 10 seconds, annealing at $50{ }^{\circ} \mathrm{C}$ for 30 seconds, and elongation at $72{ }^{\circ} \mathrm{C}$ for 30 second, followed by $72{ }^{\circ} \mathrm{C}$ for 5 minutes. PCR products were then mixed in equidensity ratios and purified with the GeneJET Gel Extraction Kit (Thermo Scientific, USA). Sequencing libraries were generated using the Illumina TruSeq DNA PCR-Free Library Preparation Kit (Illumina, USA) following manufacturer's recommendations and index codes were added. The quality of libraries was assessed using the Qubit 2.0 Fluorometer (Thermo Scientific, USA) and Agilent Bioanalyzer 2100 system. Quantified libraries were pooled according to effective concentration and expected data volume and sequenced on an Illumina HiSeq 2500 sequencing platform, generating $250 \mathrm{bp}$ paired-end reads.

\section{Data Processing and Analysis}

Demultiplexed sequences were imported into QIIME2 2018.2 (https://qiime2.org) for further analysis. The DADA2 pipeline [104] was used to pair forward and reverse reads and for quality control; phiX reads and chimeric sequences were filtered following a pooled consensus method. The resulting feature table was used to for taxonomic assignment based on the GreenGenes version 13.8 reference database by training a Naïve Bayes classifier using the QIIME2 q2-feature-classifier plugin [105,106,108,109]. De novo multiple sequence alignment was preformed using the MAFFT method. Phylogenetic trees were constructed using the FastTree2 and Midpoint Root methods [111].

Absolute count data from the GreenGenes taxonomic assignment was normalized into relative abundances at each taxonomic level and plotted using the QIIME2 taxa plugin. Community alpha and beta diversity were analyzed using phylogenetic and non-phylogenetic 
metrics through the QIIME2 diversity plugin. Diversity within each sample ( $\alpha$-diversity) was evaluated for evenness and richness based on the Shannon diversity index. The significance of the experiment day (day 0, day 1, day 2 and day 3) and FEC group (LS, MS, HS) variables on the samples' alpha diversity values was determined using a Kruskal-Wallis testing method. This method is a non-parametric version of the one-way ANOVA test and corrects for multiple testing using the Benjamini and Hochberg method [114]. Diversity between samples ( $\beta$-diversity) was estimated by calculating weighted UniFrac distances [115] from the feature table. This quantitative measure of $\beta$-diversity evaluates the presence and abundance of taxa, while also account for the phylogenetic relationships (in terms of phylogenetic branch length) between members of the taxonomic community [156]. The R software was used to further visualize data and generate PCoA plots from distance matrices using the qiime2R package [116,117]. A pairwise PERMANOVA statistical method was applied to test for significant differences between groups of samples, with 999 permutations used to calculate p-values [118]. Differences in $\beta$-diversity were evaluated for the samples grouped by experiment day (day 0, day 1, day 2, day 3) and by FEC shedding group (LS, MS, HS).

\section{Results}

\section{Fecal Egg Count Diagnostics Results Summary}

A total of 23 horses (92\%) had a positive detection of strongyle eggs from the FEC diagnostics. Animals with no observed strongyle eggs $(n=2)$ were classified as low egg shedders. A total of 11 horses (44\%) were classified as low shedders (LS), a total of 4 horses (16\%) were classified as moderate shedders (MS), and a total of 10 horses (40\%) were classified as high shedders (HS) (see tables 5 and 6 below). A total of 3 horses (12\%) had a positive ascarid detection 
count (range: 50-150 EPG); both of these horses also had positive strongyle counts and were classified as high egg shedding individuals and therefore were grouped with the HS group for comparisons.

Table 6. FEC diagnostics and shedding level classification group assignment results

\begin{tabular}{|l|l|l|l|l|}
\hline \multicolumn{1}{|c|}{ Shedding Group } & Horses (n) & Prevalence & $\begin{array}{c}\text { EPG } \\
\text { (mean) }\end{array}$ & $\begin{array}{c}\text { EPG } \\
\text { (range) }\end{array}$ \\
\hline Low Shedder & 11 & $44 \%$ & 75.0 & $0-175$ \\
\hline Moderate Shedders & 4 & $16 \%$ & 362.50 & $300-425$ \\
\hline High Shedders & 10 & $40 \%$ & 1072.5 & $625-2250$ \\
\hline
\end{tabular}

\section{$16 S$ rRNA Amplicon Sequencing Data Summary}

Demultiplexed sequences were imported into QIIME2 and sequencing data results were summarized. For the 100 samples the raw data resulted in a total of 20,823,783 sequences (table 7). The lowest sequence count $(51,616)$ belonged to the "Blank" control sample, which was then excluded from the downstream analysis.

Table 7. Summary of amplicon sequencing data generation

\begin{tabular}{|l|l|}
\hline \multicolumn{2}{|c|}{ Demultiplexed Sequence Counts Summary } \\
\hline Minimum & 51616 \\
\hline Median & 208907.0 \\
\hline Mean & 208237.93 \\
\hline Maximum & 219880 \\
\hline Total & 28023783 \\
\hline
\end{tabular}

The distribution of the sequence qualities at each position in the sequence data were visualized (figure 13) to determine if data needed to be truncated to remove low quality sequences. Due to high quality of sequences observed, reads were not truncated. Barcodes were trimmed from the 5 ' end of each read (6 bp) through the DADA2 pipeline. 


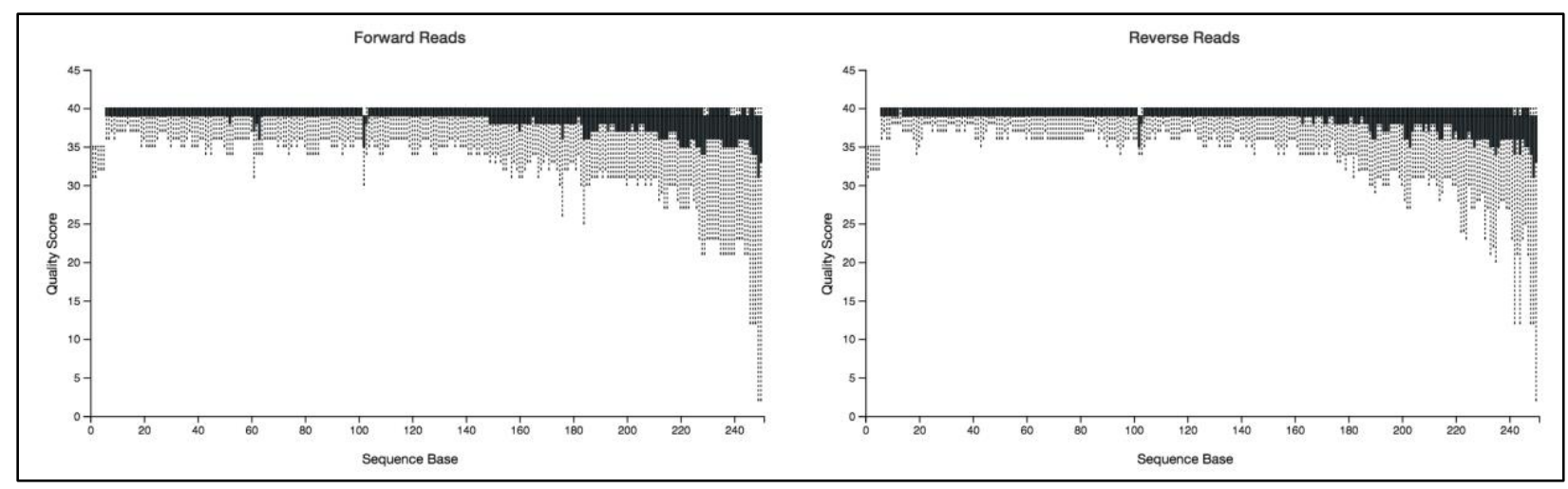

Figure 13. Distribution of sample sequencing quality

Following quality control using the DADA2 method, a feature table was constructed to map feature identifiers to representative sequences. Within the 99 samples there were 31,434 total features, with a total frequency of $6,617,513$. There was a mean frequency of $66,843.57$ (range: $52,188-78,148)$ total features per sample. For each feature, there was a mean frequency of 210.521 observations (range: 1.0-12,957). For downstream analysis, the frequency tables were rarified at an even sampling depth of 52,188 features per sample. At this threshold there were 5,166,612 of the original sequences $(78.07 \%)$ and all of the 99 samples retained in the data set. The blank sample was processed separately following the same quality control and feature table generation methods. In the single blank sample, there were 11 features identified, with a total frequency of 528 observations. For each feature, there was a mean frequency of 48.0 observations (range: 2.0138.0).

\section{Characterization of the Microbiota Between FEC Shedding Groups}

The microbial community composition and structure of day 0 fecal samples were compared between egg-shedding group. Some trends were observed in the $\alpha$-diversity measures, represented by the Shannon Diversity Index, between egg-shedding groups. The LS group had the highest average measures of $\alpha$-diversity, followed by the MS group and the HS group, which had the 
lowest average $\alpha$-diversity (see figure 14). No significant differences were observed in $\alpha$-diversity between the LS, MS, or HS sample groups (table 8).

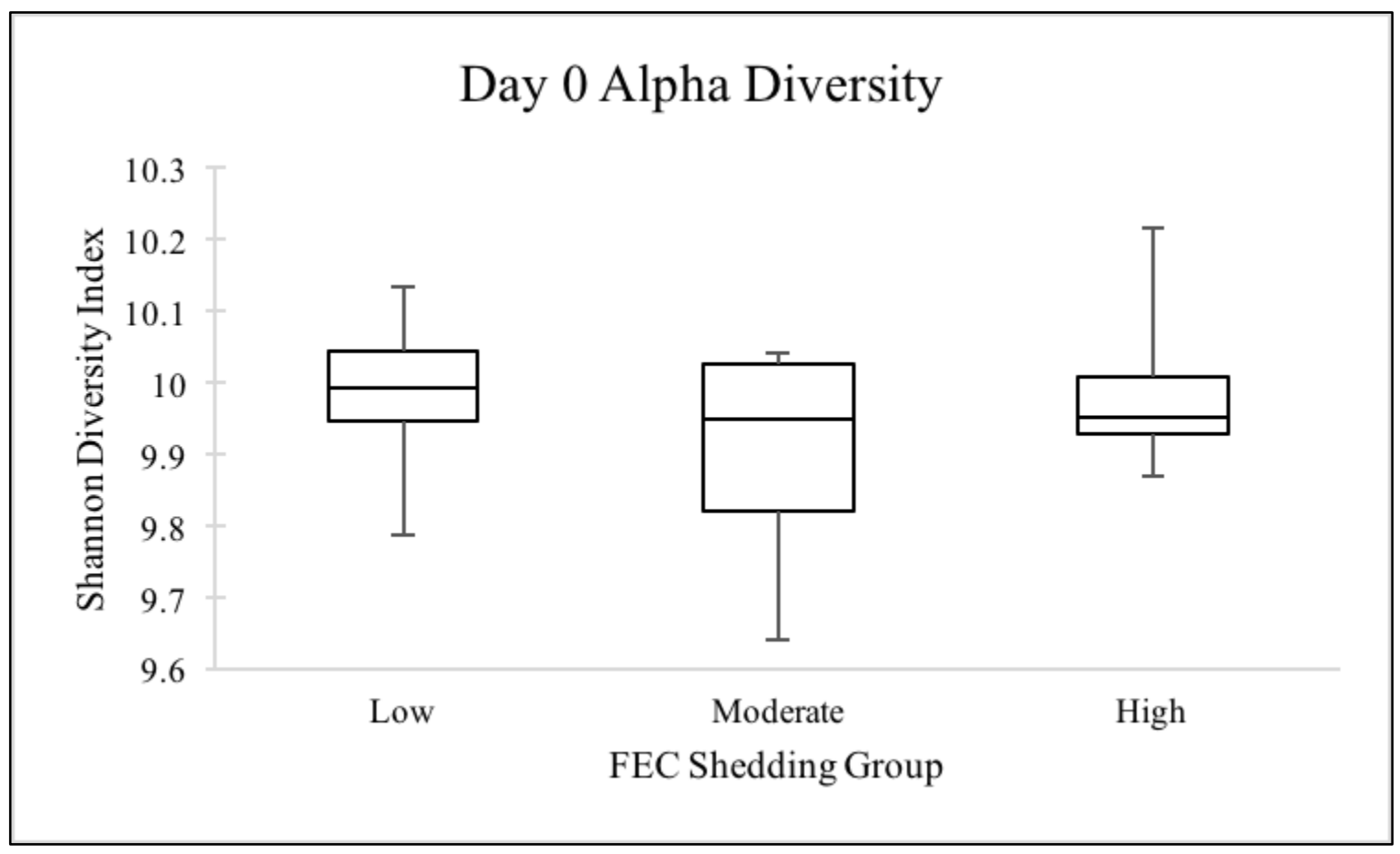

Figure 14. Alpha Diversity distribution boxplots of day 0 samples across FEC shedding groups

Table 8. Pairwise Kruskal-Wallis test results of FEC group significance based on Shannon diversity index

\begin{tabular}{llrrr} 
Group 1 & Group 2 & \multicolumn{1}{c}{ H } & \multicolumn{1}{l}{ p-value } & \multicolumn{1}{l}{ q-value } \\
LS $(\mathrm{n}=11)$ & MS $(\mathrm{n}=4)$ & 0.613636364 & 0.433421931 & 0.650132896 \\
HS $(\mathrm{n}=10)$ & LS $(\mathrm{n}=11)$ & 0.714049587 & 0.398102716 & 0.650132896 \\
HS $(\mathrm{n}=10)$ & MS $(\mathrm{n}=4)$ & 0.08 & 0.777297411 & 0.777297411
\end{tabular}

Visualization of the diversity between individual samples ( $\beta$-diversity) suggested subtle clustering associated with the FEC group assigned to each sample (see figure 15). Permutationbased statistical testing (PERMANOVA) did not indicate that the FEC group variable was a significant driver of beta-diversity differences ( $\mathrm{p}$-value $=0.182$ ). Pairwise PERMANOVA testing 
results did not indicate any significant differences ( $\mathrm{p}$-values $>0.05$ ) between individual groups of samples based on egg-shedding levels (see table 9).

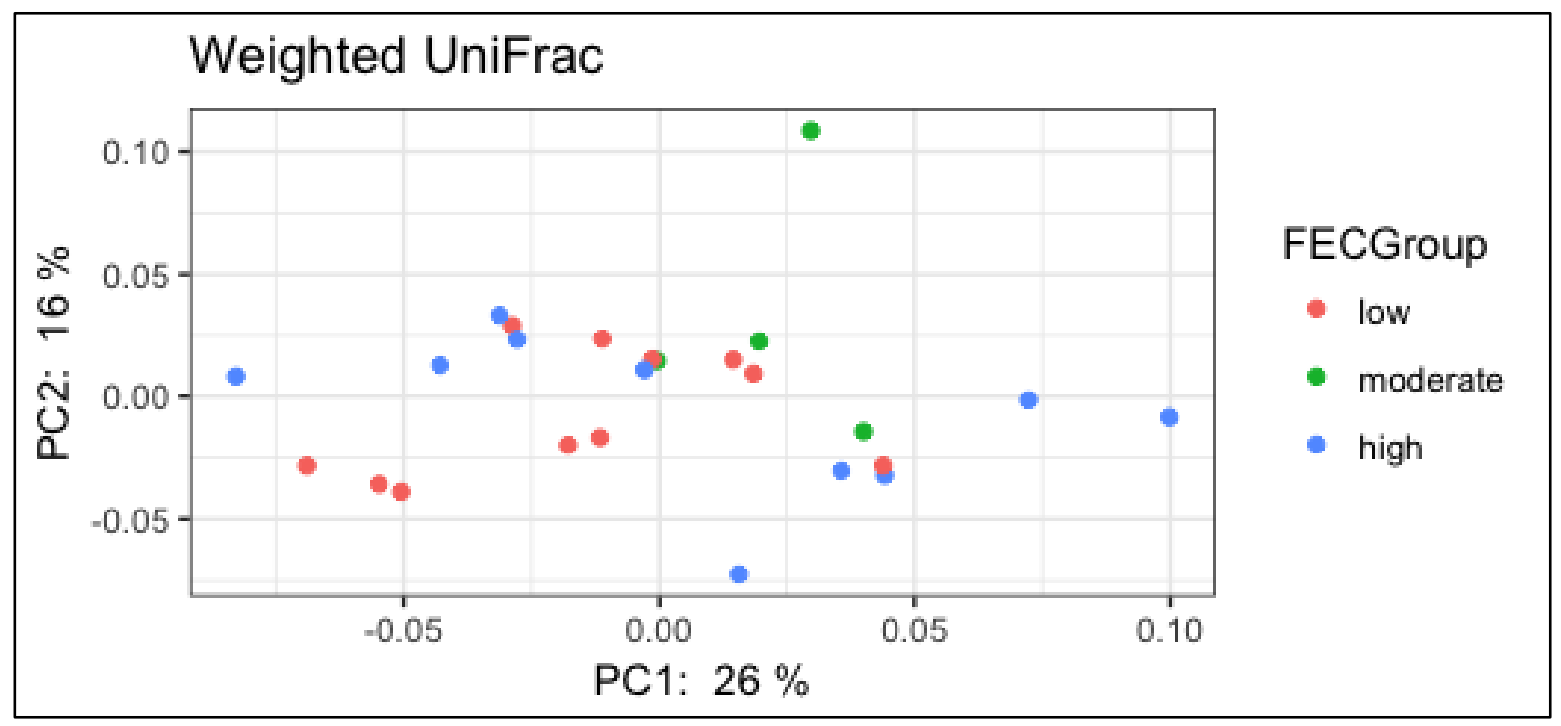

Figure 15. Weighted UniFrac PCoA labeled by FEC shedding group

Table 9. Pairwise PERMANOVA results of FEC shedding group significance based on weighted UniFrac distances

\begin{tabular}{llrrrrr} 
Group 1 & Group 2 & Sample size & Permutations & pseudo-F & p-value & \multicolumn{1}{c}{ q-value } \\
LS & MS & 15 & 999 & 1.850157467 & 0.053 & 0.159 \\
HS & LS & 21 & 999 & 1.049450182 & 0.344 & 0.361 \\
HS & MS & 14 & 999 & 1.070589281 & 0.361 & 0.361
\end{tabular}

The microbiota taxonomic profiles were compared across FEC groups (see figure 16). The Firmicutes phylum predominated across all FEC groups (LS: 44.8\%, MS: 47.1\%, HS: 47.9\%); followed by the Bacteroidetes (LS: 36.1\%, MS: 31.6\%, HS: 33.0\%); Verrucomicrobia (LS: 7.3\%, MS: 6.8\%, HS: 6.9\%); Spirochaetes (LS: 5.4\%, MS: 7.6\%, HS: 5.6\%); and the Fibrobacteres (LS: 1.8\%, MS: $2.6 \%$, HS: $1.9 \%$ ). While some minor re-ranking of the top 5 phyla was observed between FEC groups as described, the top 5 predominant phyla overall did not differentiate between the FEC groups. Sixteen other phyla were identified in the remaining percentage of sequences ( $>2 \%$ relative abundance). Results from ANCOM testing did not indicate that there 
were any significant differences in the composition of the microbiomes associated with the egg shedding level groups.

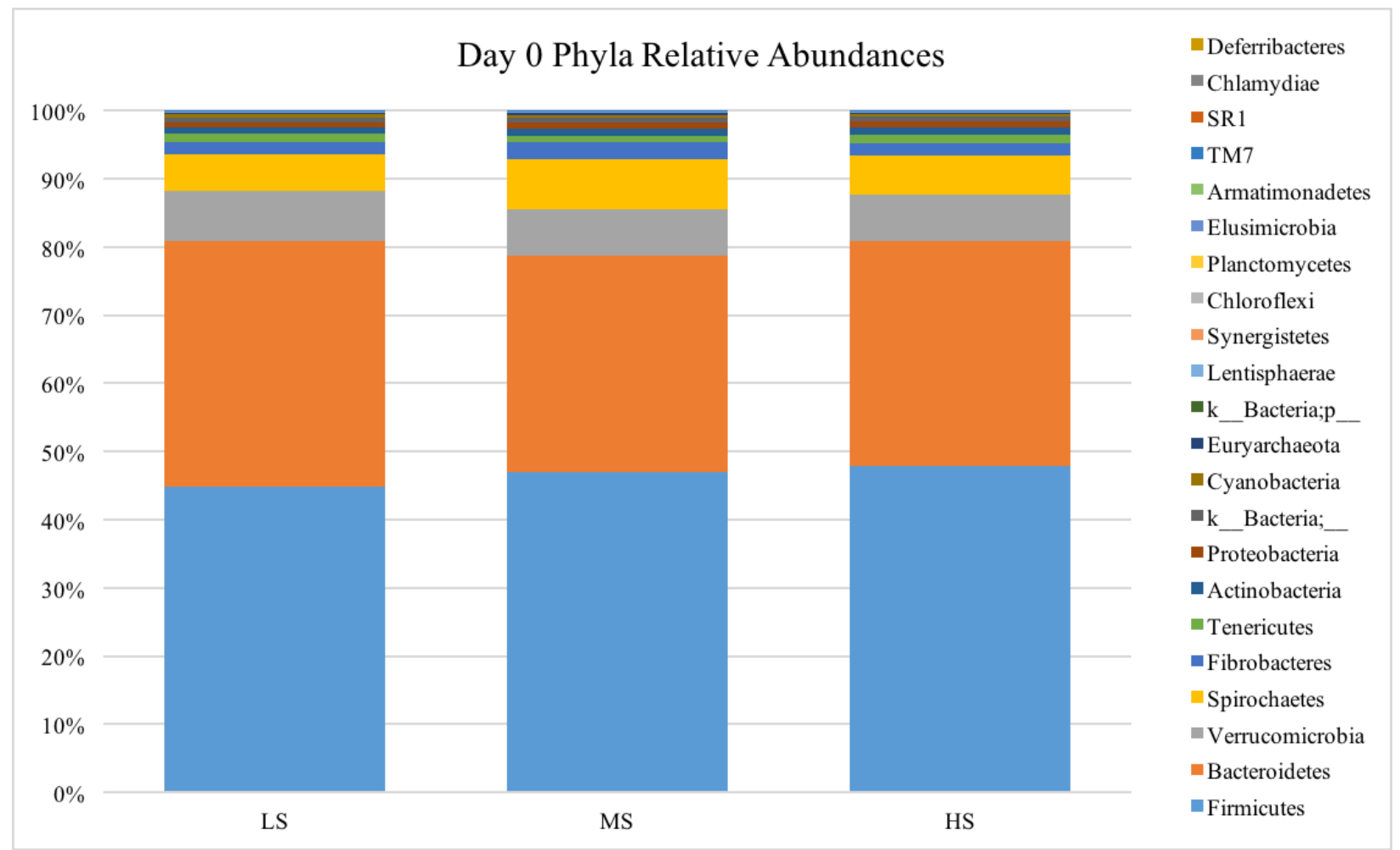

Figure 16. Observed relative abundance of phyla in day 0 (baseline) samples pooled by FEC shedding group classifications

Temporal Variation of the Microbiota Following Anthelmintic Administration

Within sample diversity ( $\alpha$-diversity) increased slightly from baseline measures established on day 0 , following anthelmintic treatment on days 1 and 2 . On day 3, there was an observable trend in decreased $\alpha$-diversity (see figure 17). Statistical testing across all experiment days did not indicate a significant difference in $\alpha$-diversity ( $\mathrm{p}$-value $=0.095$ ). Results of testing between groups of samples from each of the experiment days (table 10) indicate a significant difference in $\alpha$-diversity measures between samples from day 2 and day 3 ( $p$-value $=0.031$ ). 


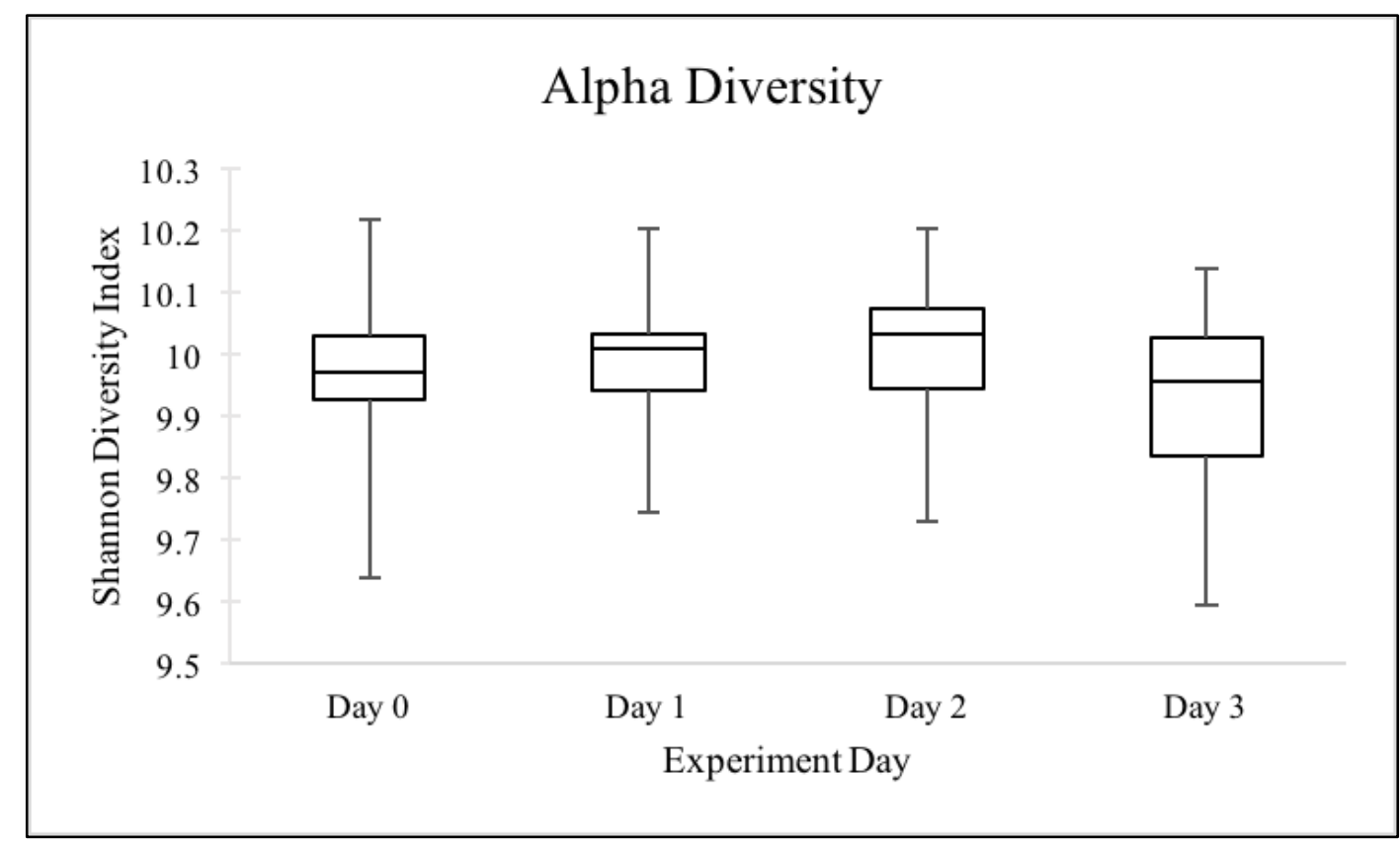

Figure 17. Alpha Diversity distribution boxplots of all samples across collected across experimental day time series

Table 10. Pairwise Kruskal-Wallis test results of experiment day group significance based on Shannon diversity index

$\begin{array}{llrrr}\text { Group 1 } & \text { Group 2 } & \text { H } & \text { p-value } & \text { q-value } \\ \text { Day 0 }(n=25) & \text { Day 1 }(n=25) & 0.849411765 & 0.3567188 & 0.3567188 \\ \text { Day 0 }(n=25) & \text { Day 2 }(n=24) & 2.4964 & 0.114106866 & 0.228213733 \\ \text { Day 0 }(n=25) & \text { Day 3 }(n=25) & 0.960094118 & 0.327163166 & 0.3567188 \\ \text { Day 1 }(n=25) & \text { Day 2 }(n=24) & 1.0816 & 0.298339901 & 0.3567188 \\ \text { Day 1 }(n=25) & \text { Day 3 }(n=25) & 2.816847059 & 0.09327947 & 0.228213733 \\ \text { Day 2 }(n=24) & \text { Day 3 }(n=25) & 4.6656 & 0.03077267 & 0.184636017\end{array}$

Principal coordinate analysis (PCoA) of $\beta$-diversity suggested some clustering of samples based on experiment day (figure 18), with stronger clustering present in samples collected on day 0. Samples collected after anthelmintic treatment (day 1, day 2, day 3) appear to be more diverse than the group of samples collected prior to treatment (day 0). 


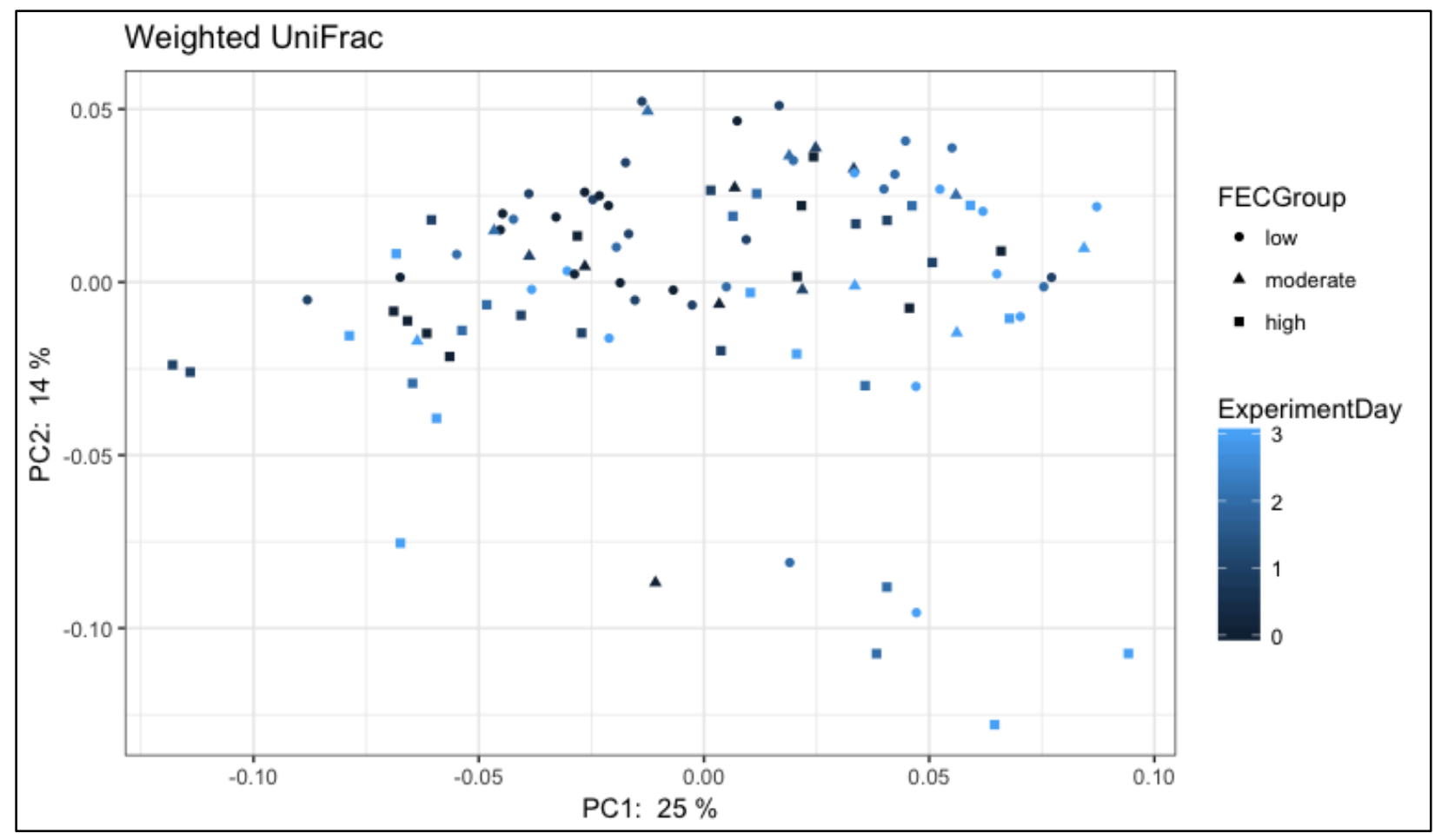

Figure 18. Weighted UniFrac PCoA labeled by FEC shedding group (shapes) and experiment day (color gradient)

The fecal microbiota community composition, represented by the weighted UniFrac metric of $\beta$ diversity, was significantly different ( $p$-value $<0.05$ ) between groups of samples collected on each experiment day, as suggested by the results of pairwise PERMANOVA, in all pairwisecomparisons except day 2 and day 3 (table 11).

Table 11. Pairwise PERMANOVA results of experiment day group significance based on weighted UniFrac distances

$\begin{array}{llrrrrr}\text { Group 1 } & \text { Group 2 } & \text { Sample size } & \text { Permutations } & \text { pseudo-F } & \text { p-value } & \text { q-value } \\ \text { Day 0 } & \text { Day 1 } & 50 & 999 & 2.640315457 & 0.009 & 0.0135 \\ \text { Day 0 } & \text { Day 2 } & 49 & 999 & 2.747850329 & 0.006 & 0.012 \\ \text { Day 0 } & \text { Day 3 } & 50 & 999 & 4.21364104 & 0.001 & 0.003 \\ \text { Day 1 } & \text { Day 2 } & 49 & 999 & 2.393543452 & 0.023 & 0.0276 \\ \text { Day 1 } & \text { Day 3 } & 50 & 999 & 3.903205051 & 0.001 & 0.003 \\ \text { Day 2 } & \text { Day 3 } & 49 & 999 & 2.085577099 & 0.05 & 0.05\end{array}$


Across all of the samples, the Firmicutes were the predominant phylum observed in the microbiota (47.2\%), followed by the Bacteroidetes (32.2\%), Verrucomicrobia (8.5\%), Spirochaetes (5.3\%), and Fibrobacteres (2.0\%). Sixteen other phyla were represented in the remaining $5 \%$ of the microbial community membership. The top 5 phyla remained constant throughout the experiment days, and no re-ranking occurred within the top 5 across the days in terms of relative abundance. Taxonomic community profiles of the samples were pooled and the relative abundance of taxonomic groups were compared across the experiment days (see figure 19). On day 2, the largest fluctuation in the relative abundance of Firmicutes was observed (D0: 46.4\%, D1: 46.0\%, D2: 49.5\%, D3: 46.9\%). The relative abundance of Bacteroidetes decreased across the experiment days (D0: $34.2 \%$, D1: 33.9\%, D2: 30.9\%, D3: 29.9\%). The abundance of Verrucomicrobia increased from day 0 to day 1, then decreased on day 2 and increased again by day 3 (D0: 7.1\%, D1: 8.7\%, D2: 7.6\%, D3: 10.5\%). The relative abundance of the Spirochaetes phylum remained relative consistent across the experiment days (D0: 5.8\%, D1: 5.2\%, D2: 5.3\%, D3: 5.6\%). Subtle changes were observed in the Fibrobacteres phylum, which initially decreased in relative abundance, and then increased on days 2 and 3 (D0: 1.9\%, D1: 1.7\%, D2: 2.1\%, D3: $2.3 \%)$ 


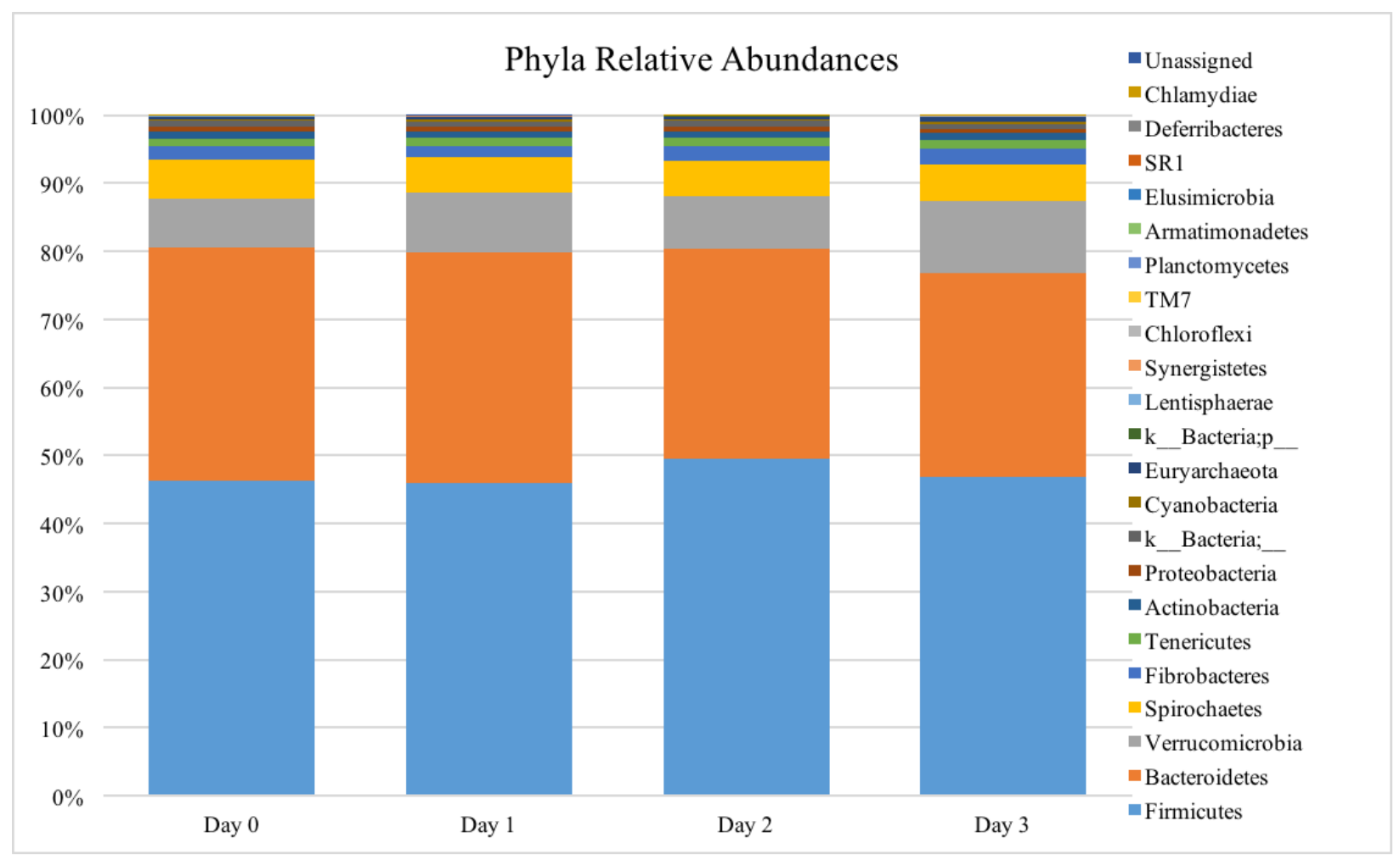

Figure 19. Observed relative abundance of phyla in all samples pooled by experiment day of which the samples were collected

Due to inconsistencies in this data, further taxonomic profile characterization and differential abundance analysis was not conducted at this time. These inconsistencies will be further described in the discussion portion of this chapter and in chapter 4.

\section{Discussion}

This study sought to provide a more comprehensive follow-up to the pilot study described in chapter II by characterizing the microbiota associated with varying helminth egg shedding levels and to characterize the temporal effect of anthelmintic drug administration on the fecal microbiota. Finally, this project also initially aimed to determine if temporal changes following anthelmintic administration were associated with the level of helminth egg shedding identified prior to 
treatment. Due to data discrepancies, these objectives were not entirely met. This discussion aims to describe what was learned by the researchers throughout this project in terms of biological significance and sources of data inconsistencies. As described further in the limitations section below, inconsistencies in sequencing data led researchers to not proceed with a full analysis of the data. The following described results and interpretations are based on preliminary analysis and results derived from microbial sequencing data should be considered cautiously in regards to the described limitations.

The first objective of this study was to characterize the fecal microbiota of horses presenting a strongyle infection. Furthermore, the study aimed to determine if there were observable difference in the microbiota community composition and structure associated with the prevalence of strongyle egg shedding, as determined by FEC.

The diagnostic results from day 0 FECs provided insight to the strongyle and ascarid egg shedding status from each individual in the sample population. Our sample population had a relatively overrepresented HS group (44\%) compared to the expected percentage (15\%-30\%) in the overall horse population [57]. The prevalence of MS individuals from our sample population (16\%) fell within the expected range (10\%-20\%), although the LS prevalence (44\%) was comparatively underrepresented (expected: 50\%-75\%). These differences are not surprising, as the previously published literature describes a multitude of factors that can explain variation in the distribution of egg shedding groups between different populations of horses $[57,157,158]$. The horses used in this study are considered "young horses" $(<5$ years of age), and it has been previously demonstrated that this age group has a significantly lower level of consistency in FEC results compared to adult and geriatric horses [157]. This age range has also demonstrated significantly higher mean strongyle egg shedding levels overall compared to other age classes 
[158]. The age of horses used in this study also likely explains the prevalence of $P$. equorum observed in 3 of the animals, as this age group falls within in the expected time in which horses are at risk for P. equorum infection [50,159]. The use of FECs to define helminth infection burdens have limitations which are described further below.

Within sample diversity ( $\alpha$-diversity) of day 0 samples was evaluated based on the Shannon diversity index. The LS group samples had the highest average $\alpha$-diversity, followed by the HS group and then the MS group which had the lowest average $\alpha$-diversity. The observed differences in average $\alpha$-diversity were not statistically different between egg shedding level groups ( $\mathrm{p}$-values $>0.05$ ). These results are in disagreement with a past study which observed a higher alpha diversity in horses with higher FEC counts, although that finding was also not found to be statistically significant [121]. This previous report compared animals based on high shedders having above 200 EPG and low shedders being anything below that threshold, which does not allow for sound comparisons to our study which had higher threshold EPG levels for cohort assignment. Other data suggest that helminth infection may lead to a decrease in alpha diversity in different model organisms $[75,76]$. To further explore and verify the impact of helminth infection on alpha diversity in horses, it would be useful to utilize more precise helminth infection diagnostic techniques, as FECs are not always representative of the true helminth burden in an animal $[59,160]$. Potential alternative methods include molecular methods, larval cultures and recently developed smart-phone egg counting applications $[59,161]$.

Some subtle differences in the relative abundance of taxonomic groups between the different FEC groups were observed. However, the results of ANCOM did not indicate that any features were significantly different in presence or abundance between the FEC groups. The small 
sample population size, and uneven distribution of animals in each experimental group used in this study may have hindered the ability to detect changes in taxonomic abundance.

The second objective of this study was the evaluate the daily temporal variation of the microbiota following anthelmintic administration. To address this objective, samples were collected at 24 hour intervals for 3 days following anthelmintic administration. This time frame was chosen because it has been previously demonstrated that the majority of the anthelmintics' active ingredients pass through the gastrointestinal tract and are excreted by the 72 hour time point following treatment $[162,163]$.

The most noticeable temporal effects of the study were observed on day 2. Samples from this collection day had the highest average alpha diversity. The alpha diversity then significantly decreased between day 2 and day 3 ( $p$-value $=0.031$ ). The significance of this result indicates that the group of samples collected on day 2 are different from those collected on day 3. This indication of difference is limited though, as the effect may be driven by complex changes in the taxonomic abundance or evenness. The results of the $\beta$-diversity analysis suggest that there was not a significant change in the presence of the dominant phylotypes in the samples between days 2 and 3. Further investigation is needed to determine what underlying mechanisms or alterations to the microbiota are driving this observed change in diversity [125,164]. However, the significant changes observed in $\alpha$-diversity suggest that this time point may be a crucial time of anthelmintic impact on the microbiota and may warrant further research focus in future time-series investigations. This is supported by previous research that has demonstrated a peak in the fecal excretion of Moxidectin at the 48-hour post-administration time point in horses [162].

On day 0 , the fecal microbiota was evaluated as a baseline for this longitudinal study and was used as a control for comparisons over time for individual animals. The taxa identified in the 
baseline samples, and in other samples throughout the experiment, are consistent with other studies in horses under similar management conditions [12]. Although, the predominant phyla identified are not consistent with previously generated data from this particular group of horses $[165,166]$. Significant differences in $\beta$-diversity associated with sample collection day suggest that differences in the presence and abundance of certain taxa may be correlated with the temporal impact of anthelmintic administration. Further analysis of taxonomic differential abundance in the community composition of samples may provide more information about the sources of taxonomic variation driving the observed differences in $\beta$-diversity. However, due to the inconsistencies in the taxa identified in this dataset, analysis into differential abundance has not yet been conducted. Further investigation into these differences will potentially be resumed once these inconsistencies have been resolved.

If possible, this study intended to further investigate any observed temporal changes that were associated with the baseline FEC shedding group classifications determined on day 0 of the study. Due to inconsistencies in the data, and the lack of notable differences observed in the shedding groups at day 0 , the analysis needed to meet this objective was dropped from the scope of this study. Future studies that include a larger sample population and animals with more drastic differences in helminth infection burden, may better allow for an assessment of the impact of anthelmintic drugs on the microbiota of horses based on their helminth burden at the time of administration. As the impact of anthelmintic on the microbiota is further investigated, this will be an important point to consider incorporating into studies. 


\section{Limitations}

When designing this experiment, we hoped to recruit more animals in the sample population without an observable helminth infection. Unfortunately, this was not the case and limited the ability to compare the microbiota of animals with and without helminth infections. Due to the low number of animals $(n=2)$ that did not have a detectable helminth infection via FEC, in addition to the higher risk of a false-negative than a false-positive result from the used FEC method [160], the animals without 0 EPG were included in the low-shedding group. Thus, a major limitation of this study was the inability to properly compare animals with and without helminth infection.

The use of fecal egg counts does not provide exact or precise representations of the helminth burden of an individual and should be interpreted with caution $[59,157,160]$. While FEC results are able to provide an approximation of egg shedding levels at a specific time point, this information does not necessarily represent the number of adult or larval stage helminths within the animal. Additionally, a relatively high level of variation between the number of eggs present in a given sample has been previously demonstrated [59]. Thus, it has been recommended that assignment into FEC shedding groups should be based on the results of multiple FEC tests performed on multiple samples from different time points. In the present study, the classification of horses into FEC groups could have been strengthened by performing more egg counts prior to the start of the study. Therefore, the use of FECs to create cohorts for comparisons in this study presented a variety of limitations that could be improved in future studies. All of the horses used in this study were managed under the same practices and were not administered any anthelmintic

products for at least 90 days prior to the start of the study. It should be noted however, that a number of management factors including previous anthelmintic administration, have been 
demonstrated to impact helminth egg shedding levels and infection rates [158]. It is possible, that exposure to differing management practices, diet, anthelmintic drugs, etc. occurred before these animals were in our care, and that these factors could have had long-term effects on the helminth infection status and/or GIM composition of the horses in this study.

Preliminary analysis of this data revealed unexplainable discrepancies in the results, due to these issues, it was decided to not move forward with further analysis until the discrepancies are resolved. Amplicon sequencing data from this project differed from past studies conducted in this research group. Samples were outsourced to a company for sequencing that had not previously been used before. This company used different quality control standards, library preparation protocols, and sequenced on a HiSeq platform rather than a MiSeq platform, generating longer reads than other studies. The FASTQ file received also still contained barcodes, which had to be manually trimmed from the reads. Initially, the quality and number of sequences per sample appeared to be far superior to previously generated data. However, following the DADA2 pipeline for quality control and sample inference, it was revealed that the vast majority of reads were identified as chimeras. Subsequently, approximately $75 \%$ of the reads from each sample were identified as chimeric and removed from the resulting feature table. A similar trend was observed in the blank sample, this sample contained only nuclease-free water although sequencing results generated 51,616 high-quality read. After running the DADA2 pipeline, only 11 features were identified in 528 total retained sequences. Taxonomic assignment did not provide resolution past the kingdom level, suggesting that these features may be associated with PCR-artifacts. The reason for this high level of data loss is yet to be determined and is being investigated by the researchers and sequencing company involved with this project at this time. It is suspected that the highsensitivity chimeric detection feature of the DADA2 pipeline identified many sequences as 
artifacts after being incorrectly joined together and subsequently removed them from the dataset. Despite the loss of data, some preliminary results were generated, following the described data analysis methods to determine if any trends could be revealed in the analysis that may explain the data loss. The taxonomic analysis of the microbiota composition revealed further surprising results. The predominant phylum identified in this data was the Firmicutes. This is consistent with the findings of data generated from some other studies in horses $[12,36,89,127,155]$. However, this is not consistent with previously generated data from this lab group in particular, in which Bacteroidetes have always been observed as the predominant phyla in equine microbiota. This finding is suspicious because the all steps of the workflow for this project are identical as those followed in the previously mentioned studies with the exception of the company used for the outsourced sequencing steps. This discrepancy further suggests that there was a technical error or bias brought on by the use of a different sequencing facility which remained to be determined. To confidentially interpret these results and identify biologically meaningful results, re-sequencing of the samples will likely be necessary. At the current time of writing, investigation into these issues are ongoing and analysis will not proceed until the reproducibility of this data can be confirmed. Unfortunately, due to the described data discrepancies, interpretation of the preliminary reported results from this study remain speculative at best.

\section{Conclusion}

The results of this study demonstrate the potential for temporal variation in the equine microbiota immediately following anthelmintic treatment. Preliminary findings suggest that changes in the fecal microbiota associated with anthelmintic administration are most evident between 48 and 72 hours following treatment. Due to inconsistencies in the data, likely from 
technical sources, the interpretation of these results remains inconclusive at the time of writing. On-going investigation into the sources of data inconsistencies may allow for re-analysis of this data in the future. 


\section{CHAPTER IV: DISCUSSION}

This thesis describes two research projects conducted to investigate the impact of anthelmintic administration on the equine microbiota. As described in chapter I, the gastrointestinal microbiota (GIM) is an essential component of mammalian health. Knowing the factors which impact and interact with the GIM is vital for the development of new medical advancements which reflect our enhanced understanding of the importance of the host-associated microbiome. This final thesis chapter will review the implications and limitations of the two previously described research and future outlooks of research pertaining to the equine-specific GIM, and the microbiota in general.

\section{Discussion of the Presented Research}

A pilot study was conducted, as described in chapter II, which investigated the effects of anthelmintic drug administration on the equine fecal microbiota. This study was unique in its' approach as it evaluated the impact of anthelmintics on animals that did not present with a helminth infection. While this approach may seem counterintuitive, the use of anthelmintic products in healthy animals has become common and is well documented $[49,57,64,160]$. The side effects of anthelmintics on uninfected animals, however, has not been investigated. More so, these potential side effects have not been evaluated in the current age of microbiome research - where substantial technological advancements have enhanced researchers' abilities to identify and understand dynamics of the microbiome. Given our recently enhanced understanding and appreciation for the importance of the GIM in health and disease, the identification of any variable which could potentially result in dysbiosis should not be taken lightly. Therefore, it is important to investigate 
factors that have the potential to influence the GIM, especially when those factors are associated with commonly used veterinary practices, such as anthelmintic use. The results of this study demonstrate that anthelmintic drugs do have an impact on the equine gastrointestinal microbiota when administered to horses without an observable helminth infection. The acute and long-term health consequences of this impact are not yet known and further investigation is needed to determine what functional changes may be occurring as a result. Due to our developing understanding of the consequences of GIM dysbiosis, these preliminary findings may provide a new body of evidence for the reasons against "routine" dew orming and the need for evidence based practices.

The second project described in this thesis was designed to expand on the results of the pilot study, while considering new questions raised by the previous findings. The results of this project were meaningful in a more ways than one. To some extent, the objectives of the study were met, and some valuable information was gained regarding the initial aims. This study focused on the temporal variation in the microbiota exhibited following anthelmintic administration. Preliminary observations indicate that the 48 hours following anthelmintic administration may have the most noticeable impact on the microbiota. This data also suggested that there may be subtle differences in the microbiota associated with FEC shedding levels. The potential for temporal variation being associated with these shedding levels will require further investigation and was beyond the scope of this research. This project, however, illuminated the complexities of microbiome research to a broad extent, and the resulting data must be interpreted with caution. The data generation and processing issues that were identified during the analysis of this study are not uncommon, and exemplify many large-scale limitations in the field of microbiome research, which require careful consideration as this rapidly evolving discipline moves forward. 
Future research pertaining to the equine microbiota and dynamics associated with helminth infection and anthelmintic administration will require far more comprehensive studies. In both of the studies described, a relatively small sample size was used (study 1 : $n=10$; study 2 : $n=25$ ). This limited the statistical power of the subsequent data analysis and may have resulted in the inability to detect subtle changes, especially at lower taxonomic levels. This limitation is further exemplified by the high level of individual variation observed in both studies, as demonstrated the by effect of individual animal being highly significant in the assessment of $\beta$-diversity. Future studies should be designed to account for this issue by utilizing a larger sample size, and by continuing to limit individual variation to the best of the investigator's ability [167]. As more factors which impact the microbiome are identified in future research, variation may also be more easily explained and accounted for in the context of specific projects. The lack of a control cohort in both of these studies also may have limited the ability to make sound comparisons and interpretation of the data [167]. Due to the longitudinal design of the experiment, however, the use of baseline samples did allow each individual to serve as their own control for time-series comparisons $[167,168]$. Although, use of a control cohort would have provided additional strength for comparisons and should be considered for future research.

As described in the previous review of literature, the interactions between host-helminthmicrobiota are multifaceted and complex and will require extensive on-going research to thoroughly understand. There are over 150 parasitic species that can infect horses [169] and potentially thousands of host-associated bacterial strains with which they could potentially interact. There interactions will clearly take a significant amount of time and research to investigate, but characterizing the equine microbiota associated with each parasitic species will be a step in the right direction. This same idea should also be extended to the multitude of anthelmintic 
products used in livestock species. Different classes of anthelmintics differ in their mode of action and may potentially impact the microbiota in a variety of ways. These differences could also be dependent on the helminth infection status of the host animal and the community composition of the microbiota in question, as each type of microorganisms may be impacted differently by the anthelmintic action. Future research should extensively investigate the many different helminth species, and corresponding anthelmintic drugs used, to determine impacts to the equine microbiota.

In order to properly understand the dynamics of a microbial ecosystem it is crucial to first be able to thoroughly identify the members of the population. In marker gene surveys, this is accomplished by assigning taxonomic information to clustered sequence reads based sequence similarity or dissimilarity. This step is performed by aligning the reads to a taxonomic database $[170,171]$. For these projects, taxonomy was assigned based on the commonly used GreenGenes database. This reference database contains phylogenetic classifications that have been submitted via $16 \mathrm{~S}$ sequences to GenBank and taxonomy proposed by independent researchers, the NCBI, and the Ribosomal Data Base Project $[171,172]$. The majority of the data which has been contributed to reference databases such as GreenGenes come from human and mouse studies, as these are the most widely published model organisms. Although, the GreenGenes reference database has not been updated since the version 13.8 release in 2013. This poses an issue when trying to use these databases to gain taxonomic information on the host-associated microbiota of less studied, or just recently studied, model organisms such as horses, that are underrepresented in reference databases [173]. As a result, the taxonomic classifications obtained when assigning taxonomy to reads is often low resolution. In our research, typically $99 \%$ of reads in a dataset were classified at the phylum level, but this resolution dropped to less than $40 \%$ at the species level. This level of taxonomic resolution is quite low compared to marker gene studies of host-associated 
microbiomes in more commonly studied organisms such as humans and mice. This exemplifies the need for more diverse and comprehensive reference databases that include less frequently studied model organisms [173]. To do so, researchers will first need to utilize methods other than marker gene surveys to describe host-associated microbes that have not yet been described and may be unique to the model organisms in question. Once more diverse resources are available, previously generated data may offer more useful information due to increased sensitivity for taxonomic identification and functionality. Ultimately, this will enhance our ability to interpret biological significance from the characterization of microbiomes. This is very evident in equine microbiota research, which has recently produced many survey type publications characterizing the microbial community under a variety of conditions but lacks the collaborative effort and resources necessary to gain much functional information from characterization studies. As a result, most of the data generated from marker gene surveys in the horse remains speculative at best and serves as a descriptor or snapshot of a microbial population at a specific point in time with inconclusive speculations regards potential implications for the health of the animal.

The equine microbiome contains a wealth of information which researchers are only beginning to understand. As practices, methods and resources for microbiome research continue to improve as will our understanding of the functionality and importance of these microbial communities in horses and throughout our living world. Further research is certainly needed to properly define dysbiosis states as it pertains to equine health, and what constitutes dysbiosis as harmful versus normal shifts in a dynamic community. This information may alter the way that we approach the management of animal health by considering microorganisms as more than just pathogens, but as members of a community which lives in a delicate balance with its' host. 


\section{Future Outlooks: Microbiome Research}

Research pertaining to the microbiome in general is plagued by a lack of standardization and multi-approach variation. Data issues which arose during the second study of this thesis project are not uncommon, and exemplify broad-scale issues that must be considered when moving forward with future microbiome research.

Interest surrounding the microbiome has advanced at a very rapid pace over the last decade. In addition to a greatly enhanced understanding and appreciation for the importance of the microbiome, the advent of NGS technologies have greatly increased the ability for researchers to quickly and cost effectively study every imaginable microbiome in existence. Innovative high throughput experimental assays and sequencing technologies have revolutionized many aspects of biological research by drastically reducing the amount of time and money needed to produce massive amounts of data. As a result, a new bottleneck has come into existence, the issue of analyzing the "big data" [174]. Sifting through such enormous data sets to gain meaningful insights requires the development of bioinformatics tools, computational resources, and expert personnel. While this data has provided the scientific community with many breakthrough findings, it has also generated a great deal of new questions and problems.

Another major limitation of all microbiome studies, especially marker gene surveys such as those described in this thesis, is the lack of comparability between studies. This is due to the large amount of variation in research approaches; from experimental design, to sample collection, DNA isolation, and sequencing protocols, to data analysis and even the interpretation of results [167]. There has been some attempts to create a set of standard protocols to address the biases resulting from biological and technical variation in data generation, such as those proposed by the Earth Microbiome Project, although these standards have not been readily utilized in less studied 
model organisms such as the horse $[11,135,167,175]$. While attempts can be made to explain discrepancies between the results of separate projects, this still does not allow for the data to be utilized for a proper comparative analysis.

Just during the two years of research (2016-2018) which contributed to this thesis, the main software used for data analysis was updated re-released over 10 times, the cost of sequencing continued to decrease, and even the terminology used to describe identified microbial organisms in marker gene surveys changed from "operational taxonomic units" to "features" $[108,176]$. While this progress exemplifies the fascinating evolution and fast pace of this field, it also demonstrates the issues of information becoming obsolete in a matter of months due to research leading to revised best practices. Now, more than ever, it is critical that standardized pipelines be implemented in microbiome studies to ensure that research is comparable and repeatable. However, it should also be taken into consideration that standardizing pipelines without a commitment to improvement could eventually result in the loss of innovation and progress in this field. Therefore, we should not discourage the development of novel approaches and methods which are crucial to further advancement. Researchers must also make a collaborative effort to publically share data and metadata associated with their data, in addition to detailed descriptions of their protocols to maximize our ability to identify sources of variation in microbiome data [167]. Such data sharing is required by most journals, but many field-specific journals do not, therefore it is the responsibility of authors and reviewers to make this information publically available. Ultimately, cooperation and collaboration throughout the scientific community will facilitate the highest level of productivity in our attempts to understand the microbial world that we live amongst. 


\section{Conclusion}

In conclusion, this thesis describes novel preliminary research into the effect of anthelmintic drugs on the equine fecal microbiota. Furthermore, this provides a basis for consideration pertaining to how common health practices may have unintended consequences. This is the first research to investigate the impact of anthelmintic administration on the microbiota horses without a helminth infection. Results from this research suggest that unnecessary anthelmintic use may have effects on the presence and abundance of microbial organisms that play important roles in equine health. This research also investigated the daily temporal impact of anthelmintic administration on animals with varying levels of infection and provided preliminary data suggesting dynamic timeassociated shifts in the microbial community following treatment. Finally, this research exemplified the challenges of microbiome research in both horses and other organisms, which is limiting the progress of this field. Further advancements in microbiome research is critical to improving equine management and health. 


\section{BIBLIOGRAPHY}

[1] Ley RE, Lozupone CA, Hamady M, Knight R, Gordon JI. Worlds within worlds:

evolution of the vertebrate gut microbiota. Nat Rev Microbiol 2008;6:776-88.

doi:10.1038/nrmicro1978.

[2] Smith K, McCoy K, Macpherson A. Use of axenic animals in studying the adaptation of mammals to their commensal intestinal microbiota. Seminars in Immunology 2007;19:59-69. doi:10.1016/j.smim.2006.10.002.

[3] Sekirov I, Russell SL, Antunes LCM, Finlay BB. Gut Microbiota in Health and Disease. Physiological Reviews 2010;90:859-904. doi:10.1152/physrev.00045.2009.

[4] Hooper LV, Littman DR, Macpherson AJ. Interactions Between the Microbiota and the Immune System. Science 2012;336:1268-73. doi:10.1126/science.1223490.

[5] Vuong HE, Yano JM, Fung TC, Hsiao EY. The Microbiome and Host Behavior. Annual Review of Neuroscience 2017;40:21-49. doi:10.1146/annurev-neuro-072116-031347.

[6] Lopetuso LR, Scaldaferri F, Petito V, Gasbarrini A. Commensal Clostridia: leading players in the maintenance of gut homeostasis. Gut Pathogens 2013;5:23. doi:10.1186/17574749-5-23.

[7] Turnbaugh PJ, Ley RE, Hamady M, Fraser-Liggett C, Knight R, Gordon JI. The human microbiome project: exploring the microbial part of ourselves in a changing world. Nature 2007;449:804-10. doi:10.1038/nature06244.

[8] Harwani D. The Great Plate Count Anomaly and the Unculturable Bacteria. International Journal of Scientific Research 2013;2. doi:10.15373/22778179/SEP2013/122. 
[9] Muegge BD, Kuczynski J, Knights D, Clemente JC, González A, Fontana L, et al. Diet drives convergence in gut microbiome functions across mammalian phylogeny and within humans. Science 2011;332:970-4. doi:10.1126/science.1198719.

[10] Cavalieri D, Collini S, De Filippo C, Di Paola M, Lionetti P, Massart S, et al. Impact of diet in shaping gut microbiota revealed by a comparative study in children from Europe and rural Africa. Proceedings of the National Academy of Sciences 2010;107:14691-6. doi:10.1073/pnas.1005963107.

[11] Costa MC, Weese JS. Understanding the Intestinal Microbiome in Health and Disease. Veterinary Clinics of North America: Equine Practice 2018;34:1-12. doi:10.1016/j.cveq.2017.11.005.

[12] Costa MC, Weese JS. The equine intestinal microbiome. Animal Health Research Reviews 2012;13:121-8. doi:10.1017/S1466252312000035.

[13] Dougal K, Harris PA, Edwards A, Pachebat JA, Blackmore TM, Worgan HJ, et al. A comparison of the microbiome and the metabolome of different regions of the equine hindgut. FEMS Microbiology Ecology 2012;82:642-52. doi:10.1111/j.1574-6941.2012.01441.x.

[14] Al Jassim RAM, Andrews FM. The Bacterial Community of the Horse Gastrointestinal Tract and Its Relation to Fermentative Acidosis, Laminitis, Colic, and Stomach Ulcers. Veterinary Clinics of North America: Equine Practice 2009;25:199-215. doi:10.1016/j.cveq.2009.04.005.

[15] Dicks LMT, Botha M, Dicks E, Botes M. The equine gastro-intestinal tract: An overview of the microbiota, disease and treatment. Livestock Science 2014;160:69-81. doi:10.1016/j.livsci.2013.11.025. 
[16] Poretsky R, Rodriguez-R LM, Luo C, Tsementzi D, Konstantinidis KT. Strengths and Limitations of 16 S rRNA Gene Amplicon Sequencing in Revealing Temporal Microbial Community Dynamics. PLOS ONE 2014;9:e93827. doi:10.1371/journal.pone.0093827. [17] Shah N, Tang H, Doak TG, Ye Y. COMPARING BACTERIAL COMMUNITIES INFERRED FROM 16S rRNA GENE SEQUENCING AND SHOTGUN METAGENOMICS. Biocomputing 2011, WORLD SCIENTIFIC; 2010, p. 165-76. doi:10.1142/9789814335058_0018.

[18] Reed KJ, Kunz IGZ, Scare JA, Nielsen MK, Metcalf JL, Coleman RJ, et al. Can fecal samples be used to inform about microbial communities of the equine hindgut? Journal of Equine Veterinary Science 2017;52:52-3. doi:10.1016/j.jevs.2017.03.041.

[19] Beckers KF, Schulz CJ, Childers GW. Rapid regrowth and detection of microbial contaminants in equine fecal microbiome samples. PLoS One 2017;12. doi:10.1371/journal.pone.0187044.

[20] Costa MC, Silva G, Ramos RV, Staempfli HR, Arroyo LG, Kim P, et al. Characterization and comparison of the bacterial microbiota in different gastrointestinal tract compartments in horses. The Veterinary Journal 2015;205:74-80. doi:10.1016/j.tvj1.2015.03.018.

[21] Ericsson AC, Johnson PJ, Lopes MA, Perry SC, Lanter HR. A Microbiological Map of the Healthy Equine Gastrointestinal Tract. PLoS One 2016;11. doi:10.1371/journal.pone.0166523.

[22] Benson AK, Kelly SA, Legge R, Ma F, Low SJ, Kim J, et al. Individuality in gut microbiota composition is a complex polygenic trait shaped by multiple environmental and host genetic factors. PNAS 2010;107:18933-8. doi:10.1073/pnas.1007028107. 
[23] Yatsunenko T, Rey FE, Manary MJ, Trehan I, Dominguez-Bello MG, Contreras M, et al. Human gut microbiome viewed across age and geography. Nature 2012;486:222-7. doi:10.1038/nature11053.

[24] Turnbaugh PJ, Hamady M, Yatsunenko T, Cantarel BL, Duncan A, Ley RE, et al. A core gut microbiome in obese and lean twins. Nature 2009;457:480-4. doi:10.1038/nature07540.

[25] Filippo CD, Cavalieri D, Paola MD, Ramazzotti M, Poullet JB, Massart S, et al. Impact of diet in shaping gut microbiota revealed by a comparative study in children from Europe and rural Africa. PNAS 2010;107:14691-6. doi:10.1073/pnas.1005963107.

[26] Alumni Association and Society of Comparitive Medicine. Large Intestinal Function. The Cornell Veterinarian, vol. 65, New York State Veterinary College, Cornell University, Ithaca, N.Y.: New York State College of Veterinary Medicine; 1911, p. 309-11.

[27] Coverdale JA. HORSE SPECIES SYMPOSIUM: Can the microbiome of the horse be altered to improve digestion?,. J Anim Sci 2016;94:2275-81. doi:10.2527/jas.2015-0056.

[28] Fernandes KA, Kittelmann S, Rogers CW, Gee EK, Bolwell CF, Bermingham EN, et al. Faecal Microbiota of Forage-Fed Horses in New Zealand and the Population Dynamics of Microbial Communities following Dietary Change. PLoS One 2014;9. doi:10.1371/journal.pone.0112846.

[29] Destrez A, Grimm P, Cezilly F, Julliand V. Changes of the hindgut microbiota due to high-starch diet can be associated with behavioral stress response in horses. Physiology \& Behavior 2015;149:159-64. doi:10.1016/j.physbeh.2015.05.039.

[30] Orlando L. Equids. Current Biology 2015;25:R973-8. doi:10.1016/j.cub.2015.09.005.

[31] Mihlbachler MC, Rivals F, Solounias N, Semprebon GM. Dietary Change and Evolution of Horses in North America. Science 2011;331:1178-81. doi:10.1126/science.1196166. 
[32] Zeder MA. The Domestication of Animals. Journal of Anthropological Research 2012;68:161-90. doi:10.1079/9780851995977.0010.

[33] Geor RJ, Harris PA. How to Minimize Gastrointestinal Disease Associated With Carbohydrate Nutrition in Horses. American Association of Equine Practioners, vol. 53, Orlando, Florida, USA: American Association of Equine Practitioners; 2007, p. 178-85 ref.52. [34] Hansen NCK, Avershina E, Mydland LT, Næsset JA, Austbø D, Moen B, et al. High nutrient availability reduces the diversity and stability of the equine caecal microbiota. Microb Ecol Health Dis 2015;26. doi:10.3402/mehd.v26.27216.

[35] Willing B, Vörös A, Roos S, Jones C, Jansson A, Lindberg JE. Changes in faecal bacteria associated with concentrate and forage-only diets fed to horses in training. Equine Vet J 2009;41:908-14.

[36] Costa MC, Arroyo LG, Allen-Vercoe E, Stämpfli HR, Kim PT, Sturgeon A, et al. Comparison of the Fecal Microbiota of Healthy Horses and Horses with Colitis by High Throughput Sequencing of the V3-V5 Region of the 16S rRNA Gene. PLOS ONE 2012;7:e41484. doi:10.1371/journal.pone.0041484.

[37] Leng J, Proudman C, Blow F, Darby A, Swann J. Understanding Intestinal Microbiota in Equine Grass Sickness: Next Generation Sequencing of Faecal Bacterial DNA. Equine Veterinary Journal 2015;47:9-9. doi:10.1111/evj.12486_19.

[38] Weese JS, Holcombe SJ, Embertson RM, Kurtz KA, Roessner HA, Jalali M, et al. Changes in the faecal microbiota of mares precede the development of post partum colic. Equine Veterinary Journal 2015;47:641-9. doi:10.1111/evj.12361. 
[39] Metcalf JL, Song SJ, Morton JT, Weiss S, Seguin-Orlando A, Joly F, et al. Evaluating the impact of domestication and captivity on the horse gut microbiome. Sci Rep 2017;7. doi:10.1038/s41598-017-15375-9.

[40] de Almeida MLM, Feringer WH, Carvalho JRG, Rodrigues IM, Jordão LR, Fonseca MG, et al. Intense Exercise and Aerobic Conditioning Associated with Chromium or L-Carnitine Supplementation Modified the Fecal Microbiota of Fillies. PLoS One 2016;11. doi:10.1371/journal.pone.0167108.

[41] Schoster A, Mosing M, Jalali M, Staempfli HR, Weese JS. Effects of transport, fasting and anaesthesia on the faecal microbiota of healthy adult horses. Equine Veterinary Journal 2016;48:595-602. doi:10.1111/evj.12479.

[42] Costa MC, Stämpfli HR, Arroyo LG, Allen-Vercoe E, Gomes RG, Weese JS. Changes in the equine fecal microbiota associated with the use of systemic antimicrobial drugs. BMC Veterinary Research 2015;11:19. doi:10.1186/s12917-015-0335-7.

[43] Nielsen MK, Reinemeyer CR. Handbook of Equine Parasite Control. 2nd ed. WileyBlackwell; 2018.

[44] Gonçalves S, Julliand V, Leblond A. Risk factors associated with colic in horses. Vet Res 2002;33:641-52. doi:10.1051/vetres:2002044.

[45] Reinemeyer CR, Nielsen MK. Parasitism and Colic. Veterinary Clinics of North America: Equine Practice 2009;25:233-45. doi:10.1016/j.cveq.2009.04.003.

[46] From The Merck Veterinary Manual, online version. Gastroinestinal Parasites of Horses 2018.

[47] Shite A, Admassu B, Abere A. Large Strongyle Parastes in Equine: A Review. Advances in Biological Research 2015;9:247-52. doi:10.5829/idosi.abr.2015.9.9559. 
[48] Lyons ET, Drudge JH, Tolliver SC. Larval Cyathostomiasis. Veterinary Clinics of North America: Equine Practice 2000;16:501-13. doi:10.1016/S0749-0739(17)30092-5.

[49] Matthews JB. An update on cyathostomins: Anthelmintic resistance and worm control. Equine Veterinary Education n.d.;20:552-60. doi:10.2746/095777308X363912.

[50] Fabiani JV, Lyons ET, Nielsen MK. Dynamics of Parascaris and Strongylus spp. parasites in untreated juvenile horses. Veterinary Parasitology 2016;230:62-6. doi:10.1016/j.vetpar.2016.11.002.

[51] Clayton HM, Duncan JL. Clinical signs associated with parascaris equorum infection in worm-free pony foals and yearlings. Veterinary Parasitology 1978;4:69-78. doi:10.1016/03044017(78)90037-7.

[52] Clayton HM. Ascarids. Recent advances. Vet Clin North Am Equine Pract 1986;2:31328.

[53] Nielsen MK. Equine tapeworm infections: Disease, diagnosis and control. Equine Veterinary Education 2016;28:388-95. doi:10.1111/eve.12394.

[54] Reinemeyer CR, Nielsen MK. Review of the biology and control of Oxyuris equi. Equine Veterinary Education 2014;26:584-91. doi:10.1111/eve.12218.

[55] From The Merck Veterinary Manual, online version. Gastroinestinal Parasites of Ruminants 2018.

[56] Drudge JH, Lyons ET. Control of internal parasites of the horse. Journal of the American Veterinary Medical Association 1966;148:378-83.

[57] Kaplan RM, Nielsen MK. An evidence-based approach to equine parasite control: It ain't the 60s anymore. Equine Veterinary Education 2010;22:306-16. doi:10.1111/j.20423292.2010.00084.x. 
[58] Anthelmintic | Define Anthelmintic at Dictionary.com n.d.

http://www.dictionary.com/browse/anthelmintic (accessed May 14, 2018).

[59] AAEP Parasite Control Subcommittee of the AAEP Infectious Disease Committee. AAEP Parasite Control Guidelines n.d.

[60] Sangster NC. Pharmacology of anthelminitic resistance in cyathostomes: will it occur with the avermectin/milbemycins? VetParasitol 1999;85:189-204. doi:10.1016/S03044017(99)00099-0.

[61] Peregrine AS, Molento MB, Kaplan RM, Nielsen MK. Anthelmintic resistance in important parasites of horses: Does it really matter? Veterinary Parasitology 2014;201:1-8. doi:10.1016/j.vetpar.2014.01.004.

[62] Zoetis. 2015 AHP Equine Industry Survey. American Horse Publications 2015. https://www.americanhorsepubs.org/equine-survey/2015-equine-survey/ (accessed May 2, 2018). [63] Peachey LE, Jenkins TP, Cantacessi C. This Gut Ain't Big Enough for Both of Us. Or Is It? Helminth-Microbiota Interactions in Veterinary Species. Trends in Parasitology 2017;33:619-32. doi:10.1016/j.pt.2017.04.004.

[64] Voris N, Donecker JM, Holland RE, Conder GA. Evidence-Based Equine Internal Parasite Control 2011:6.

[65] Hillyer MH, Taylor FGR, Proudman CJ, Edwards GB, Smith JE, French NP. Case control study to identify risk factors for simple colonic obstruction and distension colic in horses. Equine Veterinary Journal 2002;34:455-63. doi:10.2746/042516402776117746.

[66] Nielsen MK, Loynachan AT, Jacobsen S, Stewart JC, Reinemeyer CR, Horohov DW. Local and systemic inflammatory and immunologic reactions to cyathostomin larvicidal therapy 
in horses. Veterinary Immunology and Immunopathology 2015;168:203-10.

doi:10.1016/j.vetimm.2015.09.009.

[67] Reynolds LA, Finlay BB, Maizels RM. Cohabitation in the intestine: interactions

between helminth parasites, bacterial microbiota and host immunity. J Immunol 2015;195:405966. doi:10.4049/jimmunol.1501432.

[68] Glendinning L, Nausch N, Free A, Taylor DW, Mutapi F. The microbiota and helminths: sharing the same niche in the human host. Parasitology 2014;141:1255-71. doi:10.1017/S0031182014000699.

[69] Mutapi F. The gut microbiome in the helminth infected host. Trends in Parasitology 2015;31:405-6. doi:10.1016/j.pt.2015.06.003.

[70] Loke P, Lim YAL. Helminths and the microbiota: parts of the hygiene hypothesis. Parasite Immunol 2015;37:314-23. doi:10.1111/pim.12193.

[71] Charlier J, van der Voort M, Kenyon F, Skuce P, Verucruysse J. Chasing helminths and their economic impact on farmed ruminants. Trends in Parasitology 2014;30:361-7. doi:10.1016/j.pt.2014.04.009.

[72] Mantovani HC, Lopes DRG, Bento CBP, Oliveira MN de. Microbiomes Associated with Animals: Implications for Livestock and Animal Production. The Brazilian Microbiome, Springer, Cham; 2017, p. 41-63. doi:10.1007/978-3-319-59997-7_4.

[73] Lee SC, Tang MS, Lim YAL, Choy SH, Kurtz ZD, Cox LM, et al. Helminth Colonization Is Associated with Increased Diversity of the Gut Microbiota. PLoS Negl Trop Dis 2014;8. doi:10.1371/journal.pntd.0002880. 
[74] Giacomin P, Zakrzewski M, Croese J, Su X, Sotillo J, McCann L, et al. Experimental hookworm infection and escalating gluten challenges are associated with increased microbial richness in celiac subjects. Scientific Reports 2015;5:13797. doi:10.1038/srep13797.

[75] Holm JB, Sorobetea D, Kiilerich P, Ramayo-Caldas Y, Estellé J, Ma T, et al. Chronic Trichuris muris Infection Decreases Diversity of the Intestinal Microbiota and Concomitantly Increases the Abundance of Lactobacilli. PLOS ONE 2015;10:e0125495. doi:10.1371/journal.pone.0125495.

[76] Cattadori IM, Sebastian A, Hao H, Katani R, Albert I, Eilertson KE, et al. Impact of Helminth Infections and Nutritional Constraints on the Small Intestine Microbiota. PLOS ONE 2016;11:e0159770. doi:10.1371/journal.pone.0159770.

[77] El-Ashram S, Nasr IA, Abouhajer F, El-Kemary M, Huang G, Dinçel G, et al. Microbial community and ovine host response varies with early and late stages of Haemonchus contortus infection. Vet Res Commun 2017;41:263-77. doi:10.1007/s11259-017-9698-5.

[78] Parfrey LW, Jirku M, Sima R, Jalovacka M, Sak B, Grigore K, et al. A benign helminth alters the host immune system and the gut microbiota in a rat model system. PLOS ONE 2017. doi:10.1371/journal.pone.0182205.

[79] Yang C-A, Liang C, Lin C-L, Hsiao C-T, Peng C-T, Lin H-C, et al. Impact of Enterobius vermicularis infection and mebendazole treatment on intestinal microbiota and host immune response. PLoS Neg1 Trop Dis 2017;11. doi:10.1371/journal.pntd.0005963.

[80] Li RW, Li W, Sun J, Yu P, Baldwin RL, Urban JF. The effect of helminth infection on the microbial composition and structure of the caprine abomasal microbiome. Scientific Reports 2016;6:20606. doi:10.1038/srep20606. 
[81] Wu S, Li RW, Li W, Beshah E, Dawson HD, Urban JF. Worm Burden-Dependent Disruption of the Porcine Colon Microbiota by Trichuris suis Infection. PLoS One 2012;7. doi:10.1371/journal.pone.0035470.

[82] Kreisinger J, Bastien G, Hauffe HC, Marchesi J, Perkins SE. Interactions between multiple helminths and the gut microbiota in wild rodents. Phil Trans R Soc B 2015;370:20140295. doi:10.1098/rstb.2014.0295.

[83] Houlden A, Hayes KS, Bancroft AJ, Worthington JJ, Wang P, Grencis RK, et al. Chronic Trichuris muris Infection in C57BL/6 Mice Causes Significant Changes in Host Microbiota and Metabolome: Effects Reversed by Pathogen Clearance. PLoS One 2015;10. doi:10.1371/journal.pone.0125945.

[84] Ralston SL, Malinowski K, Christensen R, Breuer L. Digestion in aged horsesRevisited. Journal of Equine Veterinary Science 2001;21:310-1. doi:10.1016/S07370806(01)70068-8.

[85] Reynolds LA, Smith KA, Filbey KJ, Harcus Y, Hewitson JP, Redpath SA, et al. Commensal-pathogen interactions in the intestinal tract. Gut Microbes 2014;5:522-32. doi:10.4161/gmic.32155.

[86] Holzscheiter M, Layland LE, Loffredo-Verde E, Mair K, Vogelmann R, Langer R, et al. Lack of host gut microbiota alters immune responses and intestinal granuloma formation during schistosomiasis. Clinical \& Experimental Immunology 2014;175:246-57. doi:10.1111/cei.12230.

[87] Hayes KS, Bancroft AJ, Goldrick M, Portsmouth C, Roberts IS, Grencis RK. Exploitation of the Intestinal Microflora by the Parasitic Nematode Trichuris muris. Science 2010;328:1391-4. doi:10.1126/science.1187703. 
[88] Peachey LE, Molena RA, Jenkins TP, Di Cesare A, Traversa D, Hodgkinson JE, et al. The relationships between faecal egg counts and gut microbial composition in UK Thoroughbreds infected by cyathostomins. Int J Parasitol 2018;48:403-12. doi:10.1016/j.ijpara.2017.11.003.

[89] Clark A, Sallé G, Ballan V, Reigner F, Meynadier A, Cortet J, et al. Strongyle Infection and Gut Microbiota: Profiling of Resistant and Susceptible Horses Over a Grazing Season. Front Physiol 2018;9. doi:10.3389/fphys.2018.00272.

[90] Rosa BA, Supali T, Gankpala L, Djuardi Y, Sartono E, Zhou Y, et al. Differential human gut microbiome assemblages during soil-transmitted helminth infections in Indonesia and Liberia. Microbiome 2018;6:33. doi:10.1186/s40168-018-0416-5.

[91] Pauk K, Zadražilová I, Imramovský A, Vinšová J, Pokorná M, Masaříková M, et al. New derivatives of salicylamides: Preparation and antimicrobial activity against various bacterial species. Bioorganic \& Medicinal Chemistry 2013;21:6574-81. doi:10.1016/j.bmc.2013.08.029. [92] Macielag MJ, Demers JP, Fraga-Spano SA, Hlasta DJ, Johnson SG, Kanojia RM, et al. Substituted Salicylanilides as Inhibitors of Two-Component Regulatory Systems in Bacteria. J Med Chem 1998;41:2939-45. doi:10.1021/jm9803572.

[93] Gooyit M, Janda KD. Reprofiled anthelmintics abate hypervirulent stationary-phase Clostridium difficile. Sci Rep 2016;6. doi:10.1038/srep33642.

[94] Jarrad AM, Karoli T, Blaskovich MAT, Lyras D, Cooper MA. Clostridium difficile Drug Pipeline: Challenges in Discovery and Development of New Agents. J Med Chem 2015;58:5164-85. doi:10.1021/jm5016846. 
[95] Sirois R. Comparison of the Fecal Mircrobiota of Horses Before and After Treatment for Parasitic Helminths: Massively Parallel Sequencing of the V4 Region of the 16S Ribosomal RNA Gene. Thesis. Smith College, 2013.

[96] Daniels SP, Leng J, Ellis R, Swann JR, Moore-Colyer MJS, Proudman CJ. The effect of Moxidectin Treatment on the Equine Hind Gut Microbiome, Metabonome and Feed Fermentation Kinetics in Horses with a Very Low Parasite Burden. Equine Veterinary Education n.d.

[97] Hawrelak JA. The Causes of Intestinal Dysbiosis: A Review 2004;9:18.

[98] Carding S, Verbeke K, Vipond DT, Corfe BM, Owen LJ. Dysbiosis of the gut microbiota in disease. Microb Ecol Health Dis 2015;26. doi:10.3402/mehd.v26.26191.

[99] Vijay-Kumar M, Aitken JD, Carvalho FA, Cullender TC, Mwangi S, Srinivasan S, et al. Metabolic Syndrome and Altered Gut Microbiota in Mice Lacking Toll-Like Receptor 5. Science 2010;328:228-31. doi:10.1126/science.1179721.

[100] Innate immunity and intestinal microbiota in the development of Type 1 diabetes | Nature n.d. https://www.nature.com/articles/nature07336 (accessed May 2, 2018).

[101] Comparison of the Fecal Microbiota in Horses With Equine Metabolic Syndrome and Metabolically Normal Controls Fed a Similar All-Forage Diet. Journal of Equine Veterinary Science 2016;44:9-16. doi:10.1016/j.jevs.2016.05.010.

[102] Garrett WS, Lord GM, Punit S, Lugo-Villarino G, Mazmanian S, Ito S, et al. Communicable ulcerative colitis induced by T-bet deficiency in the innate immune system. Cell 2007;131:33-45. doi:10.1016/j.cell.2007.08.017. 
[103] The relationships between faecal egg counts and gut microbial composition in UK Thoroughbreds infected by cyathostomins. International Journal for Parasitology 2018;48:40312. doi:10.1016/j.ijpara.2017.11.003.

[104] Callahan BJ, McMurdie PJ, Rosen MJ, Han AW, Johnson AJA, Holmes SP. DADA2: High resolution sample inference from Illumina amplicon data. Nat Methods 2016;13:581-3. doi:10.1038/nmeth.3869.

[105] McCallum A, Nigam K. A Comparison of Event Models for Naive Bayes Text Classification n.d.:8.

[106] (7) Spam Filtering with Naive Bayes - Which Naive Bayes? ResearchGate n.d. https://www.researchgate.net/publication/221650814_Spam_Filtering_with_Naive_Bayes__Which_Naive_Bayes (accessed May 2, 2018).

[107] Manning CD, Raghavan P, Schütze H. Introduction to Information Retrieval 2009:569.

[108] Bokulich NA, Kaehler BD, Rideout JR, Dillon M, Bolyen E, Knight R, et al. Optimizing taxonomic classification of marker-gene amplicon sequences with QIIME 2's q2-featureclassifier plugin. Microbiome 2018;6. doi:10.1186/s40168-018-0470-z.

[109] McDonald D, Price MN, Goodrich J, Nawrocki EP, DeSantis TZ, Probst A, et al. An improved Greengenes taxonomy with explicit ranks for ecological and evolutionary analyses of bacteria and archaea. ISME J 2012;6:610-8. doi:10.1038/ismej.2011.139.

[110] Katoh K, Standley DM. MAFFT Multiple Sequence Alignment Software Version 7: Improvements in Performance and Usability. Mol Biol Evol 2013;30:772-80. doi:10.1093/molbev/mst010.

[111] Price MN, Dehal PS, Arkin AP. FastTree 2 - Approximately Maximum-Likelihood Trees for Large Alignments. PLOS ONE 2010;5:e9490. doi:10.1371/journal.pone.0009490. 
[112] Revell Liam J. phytools: an R package for phylogenetic comparative biology (and other things). Methods in Ecology and Evolution 2011;3:217-23. doi:10.1111/j.2041210X.2011.00169.x.

[113] Kruskal WH, Wallis WA. Use of ranks in one-criterion variance analysis. Journal of the American Statistical Association 1952;47:583-621.

[114] Benjamini Y, Hochberg Y. Controlling the False Discovery Rate: A Practical and Powerful Approach to Multiple Testing. Journal of the Royal Statistical Society Series B (Methodological) 1995;57:289-300.

[115] Lozupone C, Lladser ME, Knights D, Stombaugh J, Knight R. UniFrac: an effective distance metric for microbial community comparison. ISME J 2011;5:169-72. doi:10.1038/ismej.2010.133.

[116] Bisanz J. quime2R. 2018.

[117] R Core Team. R: A language and environment for statistical computing. Vienna, Austria: R Foundation for Statistical Computing; 2013.

[118] Anderson MJ. A new method for non-parametric multivariate analysis of variance. Austral Ecology n.d.;26:32-46. doi:10.1111/j.1442-9993.2001.01070.pp.x.

[119] Love MI, Huber W, Anders S. Moderated estimation of fold change and dispersion for RNA-seq data with DESeq2. Genome Biol 2014;15. doi:10.1186/s13059-014-0550-8.

[120] Richterich P. Estimation of Errors in "Raw" DNA Sequences: A Validation Study. Genome Res 1998;8:251-9.

[121] Peachey LE, Molena RA, Jenkins TP, Di Cesare A, Traversa D, Hodgkinson JE, et al. The relationships between faecal egg counts and gut microbial composition in UK 
Thoroughbreds infected by cyathostomins. Int J Parasitol 2018;48:403-12. doi:10.1016/j.ijpara.2017.11.003.

[122] Petersen C, Round JL. Defining dysbiosis and its influence on host immunity and disease. Cell Microbiol 2014;16:1024-33. doi:10.1111/cmi.12308.

[123] Manichanh C, Rigottier-Gois L, Bonnaud E, Gloux K, Pelletier E, Frangeul L, et al. Reduced diversity of faecal microbiota in Crohn's disease revealed by a metagenomic approach. Gut 2006;55:205-11. doi:10.1136/gut.2005.073817.

[124] Ott SJ, Musfeldt M, Wenderoth DF, Hampe J, Brant O, Fölsch UR, et al. Reduction in diversity of the colonic mucosa associated bacterial microflora in patients with active inflammatory bowel disease. Gut 2004;53:685-93. doi:10.1136/gut.2003.025403. [125] Shade A. Diversity is the question, not the answer. The ISME Journal 2017;11:1-6. doi:10.1038/ismej.2016.118.

[126] Ericsson AC, Johnson PJ, Lopes MA, Perry SC, Lanter HR. A Microbiological Map of the Healthy Equine Gastrointestinal Tract. PLoS One 2016;11. doi:10.1371/journal.pone.0166523.

[127] Steelman SM, Chowdhary BP, Dowd S, Suchodolski J, Janečka JE. Pyrosequencing of 16S rRNA genes in fecal samples reveals high diversity of hindgut microflora in horses and potential links to chronic laminitis. BMC Vet Res 2012;8:231. doi:10.1186/1746-6148-8-231. [128] Venable EB, Fenton K, Braner V, Reddington C, Halpin M, Heitz S, et al. Effects of Feeding Management on the Equine Cecal Microbiota. Journal of Equine Veterinary Science n.d.;49:113-21. doi:10.1016/j.jevs.2016.09.010. 
[129] Jevit MJ. Microflora of the equine gut and its ramifications on the development of laminitis; A comparison of fecal and cecal diversity and Illumina and Roche 454 sequencers n.d.:110.

[130] Watanabe Y, Nagai F, Morotomi M. Characterization of Phascolarctobacterium succinatutens sp. nov., an Asaccharolytic, Succinate-Utilizing Bacterium Isolated from Human Feces. Appl Environ Microbiol 2012;78:511-8. doi:10.1128/AEM.06035-11.

[131] Jiang W, Wu N, Wang X, Chi Y, Zhang Y, Qiu X, et al. Dysbiosis gut microbiota associated with inflammation and impaired mucosal immune function in intestine of humans with non-alcoholic fatty liver disease. Scientific Reports 2015;5:8096. doi:10.1038/srep08096. [132] Vital M, Howe AC, Tiedje JM. Revealing the Bacterial Butyrate Synthesis Pathways by Analyzing (Meta)genomic Data. MBio 2014;5:e00889-14. doi:10.1128/mBio.00889-14.

[133] Warzecha CM, Coverdale JA, Janecka JE, Leatherwood JL, Pinchak WE, Wickersham TA, et al. Influence of short-term dietary starch inclusion on the equine cecal microbiome. $\mathbf{J}$ Anim Sci 2017;95:5077-90. doi:10.2527/jas2017.1754.

[134] Kristoffersen CT. Diet Effects on the Short-Term Temporal Dynamics of the Equine Hindgut Microbiota. Norwegian University of Life Sciences, 2014.

[135] Venable EB, Bland SD, McPherson JL, Francis J. Role of the gut microbiota in equine health and disease. Anim Fron 2016;6:43-9. doi:10.2527/af.2016-0033.

[136] Gorvitovskaia A, Holmes SP, Huse SM. Interpreting Prevotella and Bacteroides as biomarkers of diet and lifestyle. Microbiome 2016;4:15. doi:10.1186/s40168-016-0160-7. [137] Larsen JM. The immune response to Prevotella bacteria in chronic inflammatory disease. Immunology 2017;151:363-74. doi:10.1111/imm.12760. 
[138] Mangalam A, Shahi SK, Luckey D, Karau M, Marietta E, Luo N, et al. Human GutDerived Commensal Bacteria Suppress CNS Inflammatory and Demyelinating Disease. Cell Reports 2017;20:1269-77. doi:10.1016/j.celrep.2017.07.031.

[139] Abramson, Bielski, Cerundolo, Huttenhower, Jose U Scher AS, Jose U Scher, Andrew Sczesnak, Randy S Longman, Nicola Segata, Carles Ubeda, Craig Bielski, Tim Rostron, Vincenzo Cerundolo, Eric G Pamer, Steven B Abramson, Curtis Huttenhower, Dan R Littman, et al. Expansion of intestinal Prevotella copri correlates with enhanced susceptibility to arthritis. ELife 2013;2:e01202. doi:10.7554/eLife.01202.

[140] Kennedy R, Lappin DF, Dixon PM, Buijs MJ, Zaura E, Crielaard W, et al. The microbiome associated with equine periodontitis and oral health. Veterinary Research 2016;47:49. doi:10.1186/s13567-016-0333-1.

[141] KINOSHITA Y, NIWA H, KATAYAMA Y, HARIU K. Dominant Obligate Anaerobes Revealed in Lower Respiratory Tract Infection in Horses by 16S rRNA Gene Sequencing. J Vet Med Sci 2014;76:587-91. doi:10.1292/jvms.13-0272.

[142] Milinovich GJ, Burrell PC, Pollitt CC, Klieve AV, Blackall LL, Ouwerkerk D, et al. Microbial ecology of the equine hindgut during oligofructose-induced laminitis. The ISME Journal 2008;2:1089-100. doi:10.1038/ismej.2008.67.

[143] Liang X, Li H, Tian G, Li S. Dynamic microbe and molecule networks in a mouse model of colitis-associated colorectal cancer. Scientific Reports 2014;4:4985. doi:10.1038/srep04985. [144] Sykora S, Brandt S. Occurrence of Treponema DNA in equine hoof canker and normal hoof tissue. Equine Veterinary Journal 2015;47:627-30. doi:10.1111/evj.12327. 
[145] Kristoffersen C, Jensen RB, Avershina E, Austbø D, Tauson A-H, Rudi K. DietDependent Modular Dynamic Interactions of the Equine Cecal Microbiota. Microbes Environ 2016;31:378-86. doi:10.1264/jsme2.ME16061.

[146] Donnell MMO, Harris HMB, Jeffery IB, Claesson MJ, Younge B, Toole PWO, et al. The core faecal bacterial microbiome of Irish Thoroughbred racehorses. Letters in Applied Microbiology 2013;57:492-501. doi:10.1111/lam.12137.

[147] Sykora S, Pieber K, Simhofer H, Hackl V, Brodesser D, Brandt S. Isolation of Treponema and Tannerella spp. from equine odontoclastic tooth resorption and hypercementosis related periodontal disease n.d.;June 2013. doi:10.1111/evj.12115.

[148] Debelius J, Song SJ, Vazquez-Baeza Y, Xu ZZ, Gonzalez A, Knight R. Tiny microbes, enormous impacts: what matters in gut microbiome studies? Genome Biol 2016;17. doi:10.1186/s13059-016-1086-X.

[149] Salonen A, Lahti L, Salojärvi J, Holtrop G, Korpela K, Duncan SH, et al. Impact of diet and individual variation on intestinal microbiota composition and fermentation products in obese men. The ISME Journal 2014;8:2218-30. doi:10.1038/ismej.2014.63.

[150] Baxter NT, Wan JJ, Schubert AM, Jenior ML, Myers P, Schloss PD. Intra- and Interindividual Variations Mask Interspecies Variation in the Microbiota of Sympatric Peromyscus Populations. Appl Environ Microbiol 2015;81:396-404. doi:10.1128/AEM.0230314.

[151] Jones RB, Zhu X, Moan E, Murff HJ, Ness RM, Seidner DL, et al. Inter-niche and interindividual variation in gut microbial community assessment using stool, rectal swab, and mucosal samples. Sci Rep 2018;8. doi:10.1038/s41598-018-22408-4. 
[152] Jenkins TP, Rathnayaka Y, Perera PK, Peachey LE, Nolan MJ, Krause L, et al. Infections by human gastrointestinal helminths are associated with changes in faecal microbiota diversity and composition. PLoS ONE 2017;12:e0184719. doi:10.1371/journal.pone.0184719.

[153] Duarte AM, Jenkins TP, Latrofa MS, Giannelli A, Papadopoulos E, de Carvalho LM, et al. Helminth infections and gut microbiota - a feline perspective. Parasit Vectors 2016;9:625. doi:10.1186/s13071-016-1908-4.

[154] Ley RE, Hamady M, Lozupone C, Turnbaugh P, Ramey RR, Bircher JS, et al. Evolution of mammals and their gut microbes. Science 2008;320:1647-51. doi:10.1126/science.1155725. [155] Dougal K, Fuente G de la, Harris PA, Girdwood SE, Pinloche E, Geor RJ, et al. Characterisation of the Faecal Bacterial Community in Adult and Elderly Horses Fed a High Fibre, High Oil or High Starch Diet Using 454 Pyrosequencing. PLOS ONE 2014;9:e87424. doi:10.1371/journal.pone.0087424.

[156] Lozupone CA, Hamady M, Kelley ST, Knight R. Quantitative and Qualitative $\beta$ Diversity Measures Lead to Different Insights into Factors That Structure Microbial Communities. Appl Environ Microbiol 2007;73:1576-85. doi:10.1128/AEM.01996-06. [157] Lester HE, Morgan ER, Hodgkinson JE, Matthews JB. Analysis of Strongyle Egg Shedding Consistency in Horses and Factors That Affect It. Journal of Equine Veterinary Science 2018;60:113-119.e1. doi:10.1016/j.jevs.2017.04.006.

[158] Relf VE, Morgan ER, Hodgkinson JE, Matthews JB. Helminth egg excretion with regard to age, gender and management practices on UK Thoroughbred studs. Parasitology 2013;140:641-52. doi:10.1017/S0031182012001941.

[159] Reinemeyer CR. Diagnosis and control of anthelmintic-resistant Parascaris equorum. Parasit Vectors 2009;2:S8. doi:10.1186/1756-3305-2-S2-S8. 
[160] Lester HE, Matthews JB. Faecal worm egg count analysis for targeting anthelminitic treatment in horses: points to consider. Equine Veterinary Journal 2014;46:139-45. doi:10.1111/evj.12199.

[161] Bredtmann CM, Krücken J, Murugaiyan J, Kuzmina T, von Samson-Himmelstjerna G. Nematode Species Identification—Current Status, Challenges and Future Perspectives for Cyathostomins. Front Cell Infect Microbiol 2017;7. doi:10.3389/fcimb.2017.00283.

[162] Afzal J, Burke AB, Batten PL, DeLay RL, Miller P. Moxidectin: Metabolic Fate and Blood Pharmacokinetics of ${ }^{14}$ C-Labeled Moxidectin in Horses. Journal of Agricultural and Food Chemistry 1997;45:3627-33. doi:10.1021/jf960981s.

[163] Cobb R, Boeckh A. Moxidectin: a review of chemistry, pharmacokinetics and use in horses. Parasit Vectors 2009;2:S5. doi:10.1186/1756-3305-2-S2-S5.

[164] Johnson KV-A, Burnet PWJ. Microbiome: Should we diversify from diversity? Gut Microbes 2016;7:455-8. doi:10.1080/19490976.2016.1241933.

[165] Kunz IGZ, Reed KJ, Metcalf JL, Coleman RJ, Coleman SJ. Determining the effects of anthelmintic drugs on the equine intestinal microbiome. Journal of Equine Veterinary Science 2017;52:53. doi:10.1016/j.jevs.2017.03.038.

[166] Martin EJ, Reed KJ, Kunz IGZ, Coleman RJ, Coleman SJ. Normal variation and changes over time in the equine intestinal microbiome. Journal of Equine Veterinary Science 2017;52:60. doi:10.1016/j.jevs.2017.03.061.

[167] Goodrich JK, Di Rienzi SC, Poole AC, Koren O, Walters WA, Caporaso JG, et al. Conducting a Microbiome Study. Cell 2014;158:250-62. doi:10.1016/j.cell.2014.06.037. 
[168] Dethlefsen L, Huse S, Sogin ML, Relman DA. The Pervasive Effects of an Antibiotic on the Human Gut Microbiota, as Revealed by Deep 16S rRNA Sequencing. PLOS Biology 2008;6:e280. doi:10.1371/journal.pbio.0060280.

[169] Watson J. Intestinal Parasites in Horses: What Every Horse Owner Should Know. The Horse Report 2007;25:16.

[170] Balvočiūtè M, Huson DH. SILVA, RDP, Greengenes, NCBI and OTT — how do these taxonomies compare? BMC Genomics 2017;18:114. doi:10.1186/s12864-017-3501-4.

[171] Santamaria M, Fosso B, Consiglio A, De Caro G, Grillo G, Licciulli F, et al. Reference databases for taxonomic assignment in metagenomics. Brief Bioinform 2012;13:682-95. doi:10.1093/bib/bbs036.

[172] DeSantis TZ, Hugenholtz P, Larsen N, Rojas M, Brodie EL, Keller K, et al. Greengenes, a Chimera-Checked 16S rRNA Gene Database and Workbench Compatible with ARB. Appl Environ Microbiol 2006;72:5069-72. doi:10.1128/AEM.03006-05.

[173] Werner JJ, Koren O, Hugenholtz P, DeSantis TZ, Walters WA, Caporaso JG, et al. Impact of training sets on classification of high-throughput bacterial 16s rRNA gene surveys. The ISME Journal 2012;6:94-103. doi:10.1038/ismej.2011.82.

[174] Miller M, Zhu C, Bromberg Y. clubber: removing the bioinformatics bottleneck in big data analyses. Journal of Integrative Bioinformatics 2017;14. doi:10.1515/jib-2017-0020.

[175] Field D, Amaral-Zettler L, Cochrane G, Cole JR, Dawyndt P, Garrity GM, et al. The Genomic Standards Consortium. PLoS Biol 2011;9. doi:10.1371/journal.pbio.1001088. [176] Caporaso JG, Kuczynski J, Stombaugh J, Bittinger K, Bushman FD, Costello EK, et al. QIIME allows analysis of high-throughput community sequencing data. Nat Methods 2010;7:335-6. doi:10.1038/nmeth.f.303. 


\section{APPENDIX I: FECAL EGG COUNT PROTOCOL}

\section{MODIFIED McMASTER'S METHOD}

Approximately fifty grams of feces were collected immediately following defecation from each horse and placed directly in individually labeled plastic bags. Samples were placed into a cooler on ice and transferred to a $4^{\circ} \mathrm{C}$ refrigerator for storage within 2 hours after collection. Samples were processed and fecal egg counts were preformed within 48 hours after collection. The McMaster's technique was used to quantify strongyles and ascarids egg counts in the fecal samples with a detection level of 25 eggs per gram (EPG). Twenty-six ml of Fecasol® (sodium nitrate solution, specific gravity of $1.2 \mathrm{~g} / \mathrm{ml}$ ) and four grams of feces were placed into a container. The solution was manually homogenized by stirring with a wooden spatula. Immediately after homogenizing, $0.5 \mathrm{ml}$ aliquots of the solution were added to each chamber of a McMaster slide (Chalex Corporation, Issaquah, WA, USA). After 10 minutes, parasite eggs were counted within each of the slide chambers under a light microscope at 10x magnification. The total number of eggs on the slide were then added and multiplied by 25 to obtain the EPG for each sample. 


\section{APPENDIX II: DNA ISOLATION PROTOCOL}

Prior to DNA isolation, samples were thawed on ice $\left(4^{\circ} \mathrm{C}\right)$ and homogenized using a sterilized spatula. Isolation was preformed using the PowerSoil ${ }^{\circledR}$ DNA Isolation Kit (MOBIO Carlsbad, California, Catalog No. 12888-50 \& 12888-100) following a modified version of the manufacturer's protocol as described below:

1. To PowerBead tubes provided, add 0.25 grams of sample.

2. Gently vortex to mix.

3. Check Solution C1. If Solution $\mathrm{C} 1$ has precipitated, heat solution to $60^{\circ} \mathrm{C}$ until dissolved before use.

4. Add $60 \mu \mathrm{l}$ of Solution $\mathbf{C 1}$ and invert several times or vortex briefly.

5. Heat the tubes at $65^{\circ} \mathrm{C}$ for 10 minutes.

6. Secure the PowerBead tubes horizontally using the MOBIO Vortex Adaptor tube holder (MOBIO Catalog No. 13000-V1-24). Vortex at maximum speed for 10 minutes.

7. Centrifuge the PowerBead tubes at $13,000 \mathrm{x}$ g for 1 minute.

8. Transfer the supernatant to a clean $2 \mathrm{ml}$ collection tube provided. Expect between $400-500$ $\mu l$ of supernatant.

9. Add $250 \mu 1$ of Solution $\mathbf{C} 2$ and vortex for 5 seconds. Incubate at $4^{\circ} \mathrm{C}$ for 5 minutes.

10. Centrifuge the tubes at $13,000 \mathrm{x}$ g for 1 minute.

11. Avoiding the pellet, transfer up to, but no more than, $600 \mu \mathrm{l}$ of supernatant to a clean $2 \mathrm{ml}$ collection tube.

12. Add $200 \mu \mathrm{l}$ of Solution $\mathbf{C 3}$ and vortex briefly. Incubate for $4^{\circ} \mathrm{C}$ for 5 minutes. 
13. Centrifuge tubes at $13,000 \mathrm{x} \mathrm{g}$ for 1 minute.

14. Avoiding the pellet, transfer up to, but no more than, $750 \mu \mathrm{l}$ of supernatant to a clean $2 \mathrm{ml}$ collection tube.

15. Shake Solution C4 to mix before use. Add $1200 \mu$ l of Solution C4 to the supernatant and vortex for 5 seconds.

16. Load approximately $675 \mu \mathrm{l}$ of supernatant onto a spin filter provided and centrifuge at $13,000 \mathrm{x} g$ for 1 minute. Discard the flow through and repeat until all the supernatant has been loaded onto the spin filter.

- A total of three loads for each sample processed are required

17. Add $500 \mu \mathrm{l}$ of Solution C5 and centrifuge for 1 minute at 13,000 x g.

18. Discard flow through.

19. Centrifuge again for 1 minute at $13,000 \times \mathrm{g}$.

20. Carefully place the spin filter in a clean $2 \mathrm{ml}$ collection tube. Avoid splashing any of Solution C5 onto the spin filter.

21. Add $100 \mu$ l of Solution C6 to the center of the white filter membrane.

- Eluting with $100 \mu \mathrm{L}$ of Solution C6 will maximize DNA yield. For more concentrated DNA, a minimum of $50 \mu \mathrm{L}$ of this solution can be used. Do not use less than $50 \mu \mathrm{L}$ of Solution C6 at this step.

22. Centrifuge at $13,000 \mathrm{x}$ g for 1 minute and discard the spin filter basket. 


\section{GLOSSARY}

Microbiota: All microorganisms present in a defined environment

Microbiome: An entire habitat, including all genes and genomes contributed by members of a microbiota and the surrounding environmental conditions

Metagenome: The collection of genes and genomes contributed by members of a microbiota

Diversity: Mathematical equation accounting for the measures of richness and evenness in a given population or populations

Alpha diversity: The diversity within a single ecosystem, site, or environment

Richness: Number of different species represented in an ecosystem or community

Evenness: Distribution of the abundance of members within an ecosystem or community

Beta diversity: The diversity differences between two or more ecosystems, sites or environments 


\section{LIST OF ABBREVIATIONS}

AAEP: American Association of Equine Practitioners

ANOVA: Analysis of variance

FEC: Fecal egg count

g: gram

GIM: Gastrointestinal microbiota

kg: kilogram

mg: milligram

NGS: Next-generation sequencing

OTU: Operational taxonomic unit

PCoA: principal component analysis

PCR: polymerase chain reaction 